\title{
Monte Carlo Simulation of Adsorption Processes on Heterogeneous Crystal Surfaces
}

\author{
by \\ Leila Rajabibonab
}

\begin{abstract}
A thesis
submitted to the Victoria University of Wellington

in fulfilment of the requirements for the degree of

Doctor of Philosophy
\end{abstract}

VICTORIA UNIVERSITY OF WELLINGTON

November 2017 



\section{Declaration of Authorship}

I, Leila Rajabibonab, declare that this thesis titled, 'Monte Carlo Simulation of Adsorption Processes on Heterogeneous Crystal Surfaces' and the work presented in it are my own. I confirm that:

- This work was done wholly or mainly while in candidature for a research degree at this University.

- Where any part of this thesis has previously been submitted for a degree or any other qualification at this University or any other institution, this has been clearly stated.

- Where I have consulted the published work of others, this is always clearly attributed.

- Where I have quoted from the work of others, the source is always given. With the exception of such quotations, this thesis is entirely my own work.

- I have acknowledged all main sources of help.

- Where the thesis is based on work done by myself jointly with others, I have made clear exactly what was done by others and what I have contributed myself.

Signed:

Date: 
"In childhood we strove to go to school,

Our turn to teach, joyous as a rule,

The end of the story is sad and cruel,

From dust we came, and gone with winds cool."

- Omar Khayyam 


\title{
VICTORIA UNIVERSITY OF WELLINGTON
}

\author{
Abstract \\ Department of Physics \\ School of Chemical and Physical Sciences
}

by Leila Rajabibonab

The simulation of adsorption processes on a heterogeneous crystal surface is the main interest of this thesis. Two applications of this event have been developed with Kinetic Monte Carlo simulation. One is how to control the crystal growth by macromolecules and the other is how to measure the effective rate of interactions near a crystal surface. The first part of this thesis, considers the effective rate of catalytic conversion on a heterogeneous catalytic surface. We assume the crystal surface has two types of active site, one is neutral and the other one is highly active. We compared our result from simulation with the analytical method that is given by the homogenization theory. Our result revealed the importance of patterns of surface energies and the size of them on reaction rate.

In the second project we consider the adsorption of a homopolymer chain on a crystal surface with two types of surface energies in order to limit the growth of one site and let the other sites grow more. We developed a new Kinetic Monte Carlo simulation method in this part, which was also applied to block copolymer chains that are more complex than a homo-polymer chain. Using this method four important phases of the polymer chains at high temperatures and also the free energies of the system across different patterns of active sites have been found. We tested different types of co-polymers to find the most differentiative block copolymer for controlling the crystal growth. 


\section{Acknowledgements}

Foremost, I would like to express my very great appreciation to Prof. Shaun Hendy for his continuous support, patience and immense knowledge. His insights helped me in all the times of research and during the writing of my thesis; without him this thesis would not have been finished.

I would also like to thank to Prof. David Williams for his great ideas all the way through my $\mathrm{PhD}$. His motivation and enthusiasm encouraged me to never feel tired. His useful guidance helped me to better understand the application of my thesis in the real world. His friendly smile will never be forgotten.

My special thanks go to Michele Governale. He is the first and one of the best supervisors at Victoria University of Wellington. I had a chance to work for him as a research assistant. Working with him made me feel a confident and curious researcher. I found working with him very productive.

Many thanks to the staff of the SCPS of the Victoria University of Wellington and School of Physics of the University of Auckland for giving me enough space to do my research in a peacful place. They were always supportive of me and made all my paperwork smooth.

I am particularly grateful for the support and good times given by my friends. During my PhD I found so many good friends and I will always miss this part. Catriona Sissons, Christin Gaedtke, Stephanie Droste, Eli Krull, Sheri Javadian, Saman Javanfard, Tanja Ottman, Jami Johnson, Sarah Fyfe, Julia Marchwicka and Thomas Haase, thanks for your emotional support during the hard times of my $\mathrm{PhD}$.

To my family, my lovely mom and dad and the best sisters in the world: Sahar, Sahere and Sepide. You were the best listeners when my project became slow, and you were the best motivators to encourage me to speed up. Thanks for being with me, making me laugh and giving me love.

To my beloved Hani Hatami for his patience and love during my PhD. I cannot begin to express my unfailing love and gratitude to him. Throughout this journey, he was the only person who has constantly supported me and encouraged me to take the next step. I had sweet and bitter days during my $\mathrm{PhD}$ life; he was with me and never left me alone. Hani you are a rock. 


\section{Contents}

Declaration of Authorship iii

Abstract $\quad$ v

Acknowledgements vi

List of Figures $\quad$ xi

List of Tables $\quad$ xv

$\begin{array}{ll}\text { Abbreviations } & \text { xvii }\end{array}$

1 Introduction $\quad 1$

1.1 Thesis outline . . . . . . . . . . . . . . . . . 5

2 Theory of Heterogeneous Catalytic Surface $\quad 7$

2.1 Introduction . . . . . . . . . . . . . . . . . . . . . . 7

2.2 Catalyst Application . . . . . . . . . . . . . . . . . . 10

2.3 Theoretical Aspects of Catalytic Surfaces . . . . . . . . . . . . . . 11

2.3.1 Physisorption . . . . . . . . . . . . . . . . . 12

2.3 .2 Chemisorption . . . . . . . . . . . . . . 12

2.3 .3 Dynamics of the Gas . . . . . . . . . . . . . . . . . . . . 13

2.3.3.1 Mean free time . . . . . . . . . . . . . . . . 13

2.3.3.2 Mean free path . . . . . . . . . . . . . . . . . 14

2.3.3.3 Collision rate . . . . . . . . . . . . . . 15

2.3.3.4 Diffusion . . . . . . . . . . . . . . . 15

2.3.4 Reaction Mechanism . . . . . . . . . . . . . . . . 17

2.3 .5 Langmuir Isotherm . . . . . . . . . . . . . . . . . . . . . . . . . . . . . . . 18

2.3.6 Homogenization Theory . . . . . . . . . . . . . . . . . . . . 19

2.3.7 Diffusion toward Heterogeneous Surface . . . . . . . . . . . . . 20

2.4 Homogenization of Heterogeneous Catalytic Surface . . . . . . . . . . . 23

2.5 Conclusion . . . . . . . . . . . . . . . . . . . . . . 29

3 Monte Carlo Simulation of the Effective Reactivity of a Heterogeneous Catalytic Surface 
3.1 Introduction . . . . . . . . . . . . . . . . . . . 31

3.2 Numerical Simulation of Diffusion Limited Catalysis . . . . . . . . . . . . . . . 32

3.2 .1 Random walk model . . . . . . . . . . . . . . . . . . 32

3.2 .2 Continuum limit . . . . . . . . . . . . . . . . . . 33

3.2.3 Kinetic Monte Carlo simulation method . . . . . . . . . . . . . . 35

3.3 Simulation of heterogeneous system . . . . . . . . . . . . 38

3.3 .1 Results . . . . . . . . . . . . . . . . . 39

3.4 Discussion and conclusions . . . . . . . . . . . . . . . . . . . 42

4 Introduction to Polymer Physics $\quad \mathbf{4 5}$

4.0.1 Overview of Polymer models . . . . . . . . . . . . . 47

$4.0 .2 \quad$ Scaling of Polymer Chain . . . . . . . . . . . . . . . 48

$4.0 .3 \quad$ Flory Exponent . . . . . . . . . . . . . . . . . . . . . . . . . 49

4.1 The Linear Polymer Chain Model . . . . . . . . . . . . . . . . . . . . 50

4.1 .1 Continuous Space Model . . . . . . . . . . . . . . . . . . . 50

4.1 .2 Discrete Space model . . . . . . . . . . . . . . . . . . . . 52

4.2 Gaussian Distribution . . . . . . . . . . . . . . . . . 52

4.3 Ideal and real polymer chain distribution . . . . . . . . . . . . . 54

4.3.1 Random Walk model of Ideal chain . . . . . . . . . . . . . . . 55

4.3.2 Distribution function of ideal polymer chain . . . . . . . . . . . 57

4.4 Excluded Volume Effect . . . . . . . . . . . . . . . . . . . . . 58

4.5 The Real Polymer Chains Model . . . . . . . . . . . . . . . . . . . . 59

4.5.1 Self-Avoiding Random Walk Model (SAW) . . . . . . . . . . . 59

4.6 Statistical Mechanics of a Polymer Chain in a Solution . . . . . . . . . . . 62

4.6.1 Series Expansion . . . . . . . . . . . . . . . . . . . 63

4.6.1.1 Umbrella sampling . . . . . . . . . . . . . . . . . . . . . . . . . 65

4.6.1.2 Expanded ensemble method . . . . . . . . . . 67

4.7 Conclusion . . . . . . . . . . . . . . . . . . . . . . . 69

5 Homopolymer Adsorption onto Heterogeneous Surfaces $\quad 71$

5.1 Applications of Polymer Adsorption . . . . . . . . . . . . . . . . . . 72

5.2 An Overview of Polymer Adsorption . . . . . . . . . . . . . . . 73

5.3 Theory of Polymer Adsorption _. . . . . . . . . . . . . . . 74

5.4 Polymer adsorption near heterogeneous surfaces by MC method . . . . . . 77

5.4.1 Free energy calculation of homopolymer chain on lattice space . . 77

5.4.1.1 Change the conformation of the chain . . . . . . . 80

5.4.1.2 Swap between sub-ensembles . . . . . . . . . . . 82

5.5 Results . . . . . . . . . . . . . . . . . . . 84

5.5.1 Probability of Desorption state of homopolymer chain onto heterogeneous surface . . . . . . . . . . . . . 85

5.5.2 Adsorption energy of homopolymer chain onto heterogeneous surface 85

5.5.2.1 An overview of four different phases of the system . . . 8 86

5.5.2.2 Expansion of homopolymer adsorption . . . . . . . 89

5.5.2.3 Critical temperature of adsorption and desorption . . . . 90

5.5.2.4 Pattern Recognition . . . . . . . . . . . . . . . . . . 92

5.5 .2 .5 Phase diagram . . . . . . . . . . . . . . 95

5.5 .2 .6 Compatible Surfaces . . . . . . . . . . . . . . . . . 97 
5.6 Conclusion . . . . . . . . . . . . . . . . . . . . . . . . 98

6 Copolymer Adsorption onto Heterogeneous Surfaces 101

6.1 Methodology . . . . . . . . . . . . . . . . . . . . . 103

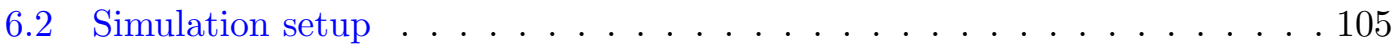

6.2.1 Adsorption Energy . . . . . . . . . . . . . . . . . . . . 110

6.2 .2 Pattern Recognition . . . . . . . . . . . . . . . . . . . 113

6.2 .3 Phase diagram . . . . . . . . . . . . . . . . . . . . . . . . . . . . . . . . . . . . . . . .

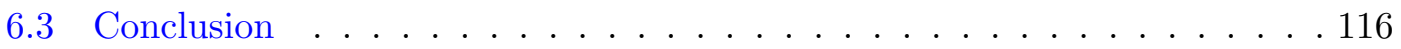

$\begin{array}{llr}7 & \text { Conclusion } & 121\end{array}$

A Homogenization theory in the Limitation of a very small L 125

$\begin{array}{ll}\text { B MC Algorithm of Chapters 4,5,6 } & 127\end{array}$

B.0.1 KMC Algorithm . . . . . . . . . . . . . . . . . 127

B.0.2 Kinetic MC algorithm of Homopolymer adsorption . . . . . . . . . 127

B.0.3 Kinetic MC algorithm of Copolymer adsorption . . . . . . . . . . . 128

$\begin{array}{ll}\text { C Adsorption concept } & 131\end{array}$

C.1 Adsorption versus Absorption . . . . . . . . . . . . . . . . . . . . . 131

$\begin{array}{lr}\text { Bibliography } & 133\end{array}$ 



\section{List of Figures}

1.1 An illustration of different types of roughness in $\mathrm{TiO}_{2}$ crystal structure. The orientation of $\mathrm{TiO}_{2}$ molecules makes different surface energies that lead to different growth rates under certain circumstances. . . . . . . . 3

2.1 This figure shows the role of the catalyst during a chemical reaction. The catalyst is an additive material and does not need to be in a chemical reaction, however, it can increase the rate of reaction significantly. From left to the right shows the steps of the process, from collision to producing a product. $E_{1}$ is the energy required for this chemical process without a catalyst. $E_{2}$ is the energy required for making chemical bond with the surface by the presence of catalyst. . . . . . . . . . . . . . 8

2.2 This figure shows all the phases of a chemical process when reactant gas $A_{g}$ converts to particle $C_{g}$. When reactant $A_{g}$ becomes stable and continues to make a bond with the molecules on the surface, it becomes $A_{i}$. Once the chemical bond between surface and $A_{i}$ created, the new particle $C_{i}$ is made. After a while, a new particle becomes free to go and leave the

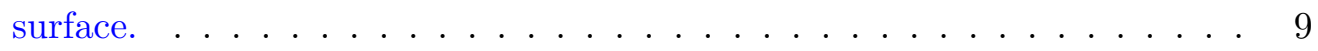

2.3 An illustration of different types of defect on nonuniform surfaces. . . . . 11

2.4 The collision of moving molecule with other random molecules. The volume amount of a tube in which particles collide with each other in the cross section of this tube. A is the cross-section for the scattering of the particle and $d$ is the radial distance of the path. $\bar{v} t$ is the path distance before the collision occurs. . . . . . . . . . . . . . . . 16

2.5 This figure demonstrates coverage versus Pressure. As the pressure increases the coverage will increase up to saturation level. Coverage has a direct relationship with the rate of interaction as well. The pressure varies as does the surface coverage. . . . . . . . . . . . . 20

2.6 Density distribution along the z-axis. Due to the heterogeneity of the surface, the distribution of density that changes linearly as a fraction of the height times the normal density on a flat surface, it approximately changes with this ratio for a heterogeneous surface. This exhibits the presence of additional term that could approximately estimate the distribution of the density over a heterogeneous surface. . . . . . . . . . . . . . . 22

2.7 (An illustration of a catalyst surface, showing active sites (black) and non-active sites (grey) distributed in a pattern of period $L$ with adsorbed particles (white) and non-absorbed particles (black). . . . . . . . . 23 
3.1 The random walker pathway in one-dimensional space. This figure shows the walker first started from the origin. His first four steps randomly were in the right direction and his next two steps were in the left direction. The right direction is assigned as the positive direction and the left direction is assigned as the negative direction. . . . . . . . . . . . 33

3.2 Time of occurrence of each event based on time the interval. Three different types of process $\left(r_{1}, r_{2}, r_{3}\right)$ happen at different times. Some event e.g $r_{3}$ takes longer than $r_{2}$ to be completed as an event. . . . . . . .

3.3 The figure compares the effective activities for a range of different domain sizes as a function of the surface coverage with effective reactivity predicted by equation (2.39)and surface coverage of active sites with $k_{1}=1.0: 0$ of $\phi=25 \%$ and $k_{2}=0.25$ elsewhere as $\tau_{c}$ is varied. For length scales $L \sim \lambda$, the effective activity exceeds that predicted by (2.39) but as $L$ gets larger, the effective activity is well described by equation (2.39).

3.4 The graph compares the effective activities for a range of different domain sizes as a function of the surface coverage with effective reactivity predicted by equation (2.42) and surface coverage of active sites with $k_{1}=$ $1.0: 0$ of $\phi=25 \%$ and $k_{2}=0.15$ elsewhere as $\tau_{c}$ is varied. For length scales $L \sim \lambda$, the effective activity exceeds that predicted by (2.42) but as $L$ gets larger, the effective activity is well described by equation (2.42).

3.5 The relationship is shown between the effective activity $k_{\text {eff }}$ and the domain size $L$ is shown for the case where $k_{1}=0.8$ at $50 \%$ coverage (and $k_{1}=0$ elsewhere) and the case where $k_{1}=1.0$ at $25 \%$ coverage. The patterns here have a checkerboard character. The effective activity in the simulations approaches that predicted by equation (2.42) as $\lambda / L \rightarrow 0$ but exceeds this as $L \rightarrow \lambda \ldots \ldots \ldots \ldots \ldots \ldots \ldots$

3.6 The figure shows the effective activity of different patterns with a surface coverage of active sites with $k_{1}=1.0$ of $\phi=25 \%$ and $k_{1}=0.0$ elsewhere as $\tau_{C}$ is varied. In the range of $0<\tau_{C} \ll \tau_{D}$, the larger length length scale regions approach agreement with equation (2.42). For the patterns with periods comparable to the mean free path $(\lambda)$, the effective activity exceeds the limit predicted by equation $(2.42)$ as $\tau_{C} \rightarrow \tau_{D} \ldots \ldots \ldots$

3.7 The figure shows the effective activity of different patterns with a surface coverage of active sites with $k_{1}=0.8$ of $\phi=50 \%$ and $k_{1}=0.0$ elsewhere as $\tau_{C}$ is varied. In the range of $0<\tau_{C} \ll \tau_{D}$, the larger length length scale regions approach agreement with equation (2.42). For the patterns with periods comparable to the mean free path $(\lambda)$, the effective activity exceeds the limit predicted by equation $(2.42)$ as $\tau_{C} \rightarrow \tau_{D} \ldots \ldots \ldots$

4.1 A general structure of single (a), branched (b) and cross-linked polymer(c). 46

4.2 This figure shows the periodic repetition of the single polymer chain that can be homopolymer (only one type of monomer), diblock copolymer (two types of monomer) or (triblock polymer). . . . . . . . . . . . .

4.3 Based on the bond length between monomers and their flexibilities, we can construct each polymer chain with these three types of models Figure (a) is a bead-stick model which models a rigid polymer chain Figure (b) is a bead-spring model which is a model for a semiflexible polymer chain. Figure (c) is pearl-necklace model and is suitable for a flexible polymer chain. . . . . . . . . . . . . . . . . . 
4.4 This Figure shows end-to-end vector, the centre of mass and the radius of gyration in a single polymer chain. . . . . . . . . . . . . . . 49

4.5 Figure (a) shows the real polymer chain, where the two monomers cannot be closer than a specific length and Figure (b) shows the ideal polymer chain where two monomers can cross over each other. . . . . . . . . . 54

4.6 This figure shows a surface with the striped pattern of sticky patches. The monomer can adsorb on those patches. Blue stripes show the sticky sites. Once a couple of monomers adsorb on blue stripes the movement of monomer become more restricted. By applying right conformation method we can improve the simulation performance. . . . . . . . . . . . 61

4.7 These figures show the changing places of the monomers. Dash circles are the new places of the monomers and dash lines show the breaking and making new bonds. The new chain structures show on the right side of picture. Figure (a) shows Reptation algorithm where one end of the polymer chain is moved to the other end. Figure (b) shows the first monomer of the displaced part is rotated 90 degrees to the new place with the rest of the chain. Figure (c) shows two different types of kink-jump displacement, which are all in the range of the BFM length. . . . . . . . 63

4.8 Free energy barriers of transition from $\mathrm{z}$ to $\mathrm{z}^{\prime} \ldots \ldots \ldots 66$

5.1 This figure shows the bond fluctuation of a polymer chain within a cube. The dash lines show the available places that one monomer can choose from. The red circle is the fixed monomer and dashed gray circles show the new possible position of monomer. Maximum displacement is a cube

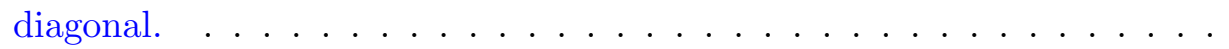

5.2 A state before conformation change is shown. $\mathrm{i}$ is the selected monomer and it is close to the end of the polymer chain, therefore in the second picture monomer makes a new bond with n-th monomer and looses its other bond with $\mathrm{i}+1$. Now $\mathrm{N}$ becomes $\mathrm{i}+1$-th monomer and the previous neighbor will become the last monomer. . . . . . . . . . . . . . 82

5.3 Centre of mass in the $\mathrm{z}$ direction for active site $\mathrm{L}=16 \ldots \ldots$. . . . . 87

5.4 Centre of mass in the $\mathrm{z}$ direction for active site $\mathrm{L}=16 \ldots \ldots$. . . . . . . . 88

5.5 Different snapshots of one arbitrary random pattern (does not change over time) at different range of temperatures: very high $=($ a) to low temperature $=(\mathrm{d})$ From a to $\mathrm{d} \beta=0.0 \epsilon^{-1}, 1.8 \epsilon^{-1}, 2.5 \epsilon^{-1}, 4.0 \epsilon^{-1} \ldots \ldots$. . . . 89

5.6 Centre of mass in the $\mathrm{z}$ direction for active site $\mathrm{L}=16 \ldots$. . . . . . . . . 90

5.7 The energy of adsorption for different patterns with a different size of the sticky island. From this figure, we can find the critical temperature of adsorption for different types of adsorption sites. Once a few monomers start to become adsorbed on the surface we measure this temperature as a critical temperature of adsorption. The $\phi$ values of striped and checkerboard pattern are the same. . . . . . . . . . . . . . . . 92

5.8 This figure shows the probability of desorption for different patterns with a different size of the attractive sites. From this figure, we can find the critical temperature of desorption for different types of attractive sites. Once a few monomers start to drop on the surface the total number of desorbed states start to become decreased and we can measure the temperature as a critical temperature of desorption. $\phi$ values of checkerboard and striped pattern are the same. . . . . . . . . . . . . 93 
5.9 Our Result and the example . . . . . . . . . . . . . . . . . . . 94

5.10 Centre of mass in the $\mathrm{z}$ direction for active site $\mathrm{L}=16 \ldots \ldots 96$

5.11 Compatible schematic to show the competition between striped and checked pattern for adsorbing polymer chain. . . . . . . . . . . . . . 97

5.12 The histogram of adsorption of Polymer chain on a mixed surface demonstrates that stripe pattern is more desirable than checkerboard for polymer to become adsorbed. . . . . . . . . . . . . . . . . . . .

6.1 Figure (a) shows diblock copolymer adsorption with the least effective design. In this type of design, the orientation of monomer type B and monomer type A are such that both can equally distribute over the surface. In Figure (b) monomer type, A and B cannot equally adsorb on the surface and adsorption of type A (green cube) will be preferred to type B (orange cube). . . . . . . . . . . . . . . . . . . . . 102

6.2 Average number of bridges for monomer type A with the strength of 0.75106

6.3 Centre of mass in the $\mathrm{z}$ direction for active site $\mathrm{L}=16 \ldots . . \ldots 107$

6.6 Centre of mass in the $\mathrm{z}$ direction for active site $\mathrm{L}=4$. . . . . . . . . . 109

6.7 Centre of mass in the $\mathrm{z}$ direction for active site $\mathrm{L}=16 \ldots . . \ldots 110$

6.8 Adsorption energy of monomers type $\mathrm{A}$ with the strength of adsorption $\epsilon_{A}=0.75$ and monomers type $\mathrm{B}$ with the strength of adsorption $\epsilon_{B}=0.25$ with the length of adsorption $\mathrm{L}=16 \ldots \ldots \ldots \ldots \ldots \ldots \ldots$. . . . . . . . . . .

6.9 Adsorption energy of monomers type $A$ with the strength of adsorption $\epsilon_{A}=0.75$ and monomers type $\mathrm{B}$ with the strength of adsorption $\epsilon_{B}=0.25$ with the length of adsorption $\mathrm{L}=16 \ldots \ldots \ldots \ldots \ldots \ldots$

6.11 This figure shows the phase diagram of the most selective design of copolymer AABAA in striped pattern as a function of length of sites and temperature. Phase (a) system is at very high temperature, phase (b) system starts to become expanded and adsorbed on the surface, phase (c) chain is become fully adsorbed). The width of the lines show that the boarder between two regions is not very sharp and it changes from case to case. Therefore, we cannot separate two phases with a certain point . . . . . . 115

6.12 This figure shows the phase diagram of the most selective design of copolymer AABAA in checkerboard pattern as a function of length of sites and temperature. Phase (a) system is at very high temperature, phase (b) system starts to become expanded and adsorbed on the surface, phase (c) chain is become fully adsorbed. The width of the lines show that the boarder between two regions is not very sharp and it changes from case to case. Therefore, we cannot separate two phases with a certain point . . 116

6.4 Centre of mass in the $\mathrm{z}$ direction for active site $\mathrm{L}=16 \ldots \ldots . . \ldots 118$

6.5 Centre of mass in the $\mathrm{z}$ direction for active site $\mathrm{L}=16 \ldots . . \ldots 119$

6.10 The average number of bridges comparison between two patterns: striped and checkerboard . . . . . . . . . . . . . . . . 120 


\section{List of Tables}

5.1 Striped pattern with size of islands $L=2$. The first column shows the $\beta$ segmentations. The second column computed the total potential energy of the system. The third column shows the probability of the subensemble occurrence in equilibrium. Three columns of $\beta_{m} F_{m}^{0} / L, \beta_{m} F_{m}^{1} / L$ and $\beta_{m} F_{m}^{2} / L$ show 3 times running to find the optimal value of free energy difference of subensembles. . . . . . . . . . . . . . . 86 



\section{Abbreviations}

EEM Extended Ensemble Method

KMC Kinetic Monte Carlo

FJC Freely Jointed Chain

MD Molecular Dynamics

SAW Self-Avoiding random Walk

BFM Bond Fluctuation Model

WLC Worm-Like Chain 

I dedicated this thesis to my mom and dad. Love you. 



\section{Chapter 1}

\section{Introduction}

We are all familiar with crystals. The most common examples are sugar, salt, and diamond; these are the products of crystallization processes[1]. Crystallization is an important process in many chemical and biological systems[2]. It happens during the purifying of a solution by making a solid layer on a surface. The resultant shape of the crystal is an important outcome of this process, so the question that will arise is how can we grow and control the crystal shape during this process in order to make an optimal crystal shape. The answer is related to the control of facet growth during crystallization[3]. Usually, during crystal growth, there are some facets that grow faster than other facets due to their surface energies[4]. Facets with a high energy surface grow faster than facets with lower energies. This will lead to a preferred orientation for the nucleation on a surface, and therefore, facets can provide specific orientation for adatoms to adsorb onto; sometimes this orientation has a periodic pattern such as a step or a terrace or, at other times, it is random such as random defects or vacancies are created during crystallization[5].

Generally, understanding the properties of patterned surfaces is the main interest of this thesis, and controlling crystal growth is one of its applications. Specifically, the subjects that we considered in the patterned surface are first studying the effective rate of adsorption of certain patterns and comparing the effective rate of these patterns together[6]. From a chemical point of view, the bond between molecules or atoms in the surface plays a key role in the interaction of the absorbing molecules and the surface. When the bonds between the absorbing molecules in the surface are strong, it is hard for 
molecules to adsorb on that facet, while in weak bond interaction molecules can attach to that facet easily[7]. This is related to the physical structure of the surface as well as rough surfaces which have many dangling bonds and it is easy for adatom to make a new bond with the surface rather than a smooth surface where this attachment can hardly occur[8].

Figure. 1.1 shows an example of the crystalline structure of $\mathrm{TiO}_{2}$. Different facets of $\mathrm{TiO}_{2}$ have different orientations, which result in different growth rates during crystal growth. The size of active sites in [001] is in the order of $10^{-7}[\mathrm{~m}]$, which is comparable to the mean free path of the ideal gas whereas the size of active sites align [011] face is somewhat broader[9]. In this direction, a group of small active sites are close together and they make an island, the size of which is multiple times bigger than a unit size. Sometimes the direction of these islands are ordered and they obey a periodic pattern. For example, islands in [011] make a striped pattern. In the case of defects or vacancies in the system, the adatom will face random patterns of highly active sites. In this example, active sites in the direction of [10̄1] have been shown as random patterns. Sometimes, a few active sites stick together and make an island, and at other times they have the same size as the mean free path of the ideal gas, which is the smallest size of active site that we study in this thesis.

Understanding the rate of interactions in different facets can help us to control the crystal growth[10]. The goal of this thesis is both to understand how the features of a crystal surface can determine its properties, but also to investigate how different crystal facets may be stabilized by the adsorption of complex molecules[11]. In order to progress towards this goal, we need to study how the adsorption of polymer onto a surface can control the crystal growth. Consequently, we can make a new crystal structure for a surface that some facets grow faster than others with a certain rate. In order to understand how to control the crystal growth we need to first study the effective rate of heterogeneous catalytic surface. We have developed a new theoretical approach based on homogenisation and applied numerical methods to study the interaction rate on crystal surfaces[12].

The Kinetic Monte Carlo method can be used to simulate adsorption process, which we will extensively discuss in chapter 3,5 and 6 . 


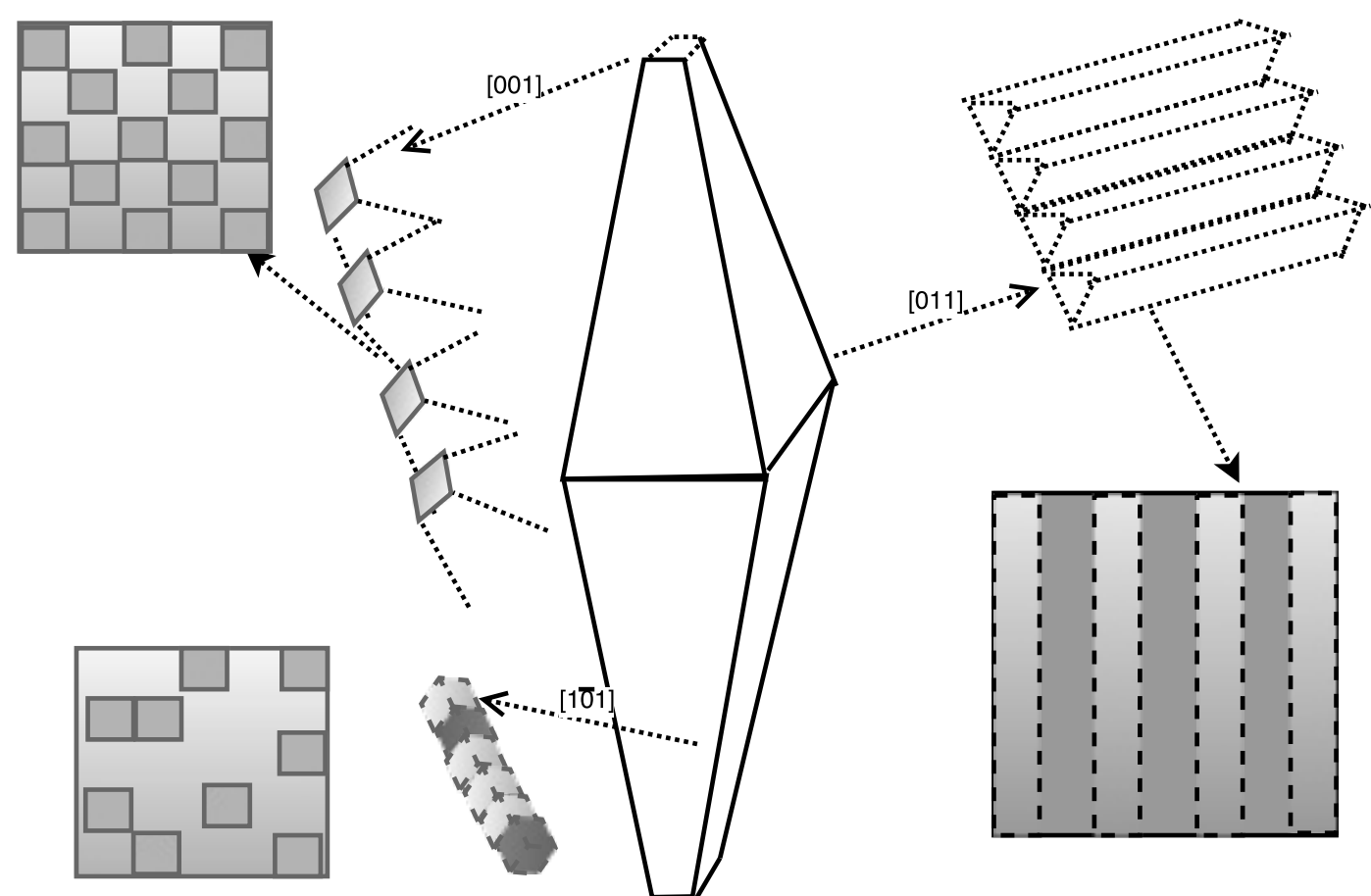

Figure 1.1: An illustration of different types of roughness in $\mathrm{TiO}_{2}$ crystal structure. The orientation of $\mathrm{TiO}_{2}$ molecules makes different surface energies that lead to different growth rates under certain circumstances.

The reactivity of the material is the subject of many chemical and biological processes. Investigating the behavior of the system at a molecular level is difficult experimentally; instead computational methods are powerful tools for understanding microscopic scales. The Monte Carlo simulation is one the useful tools that helps us to study very stochastic processes. Usually, we apply the Monte Carlo simulation to systems that we want to study in the equilibrium state. For time dependent processes like crystal growth, we can use Kinetic Monte Carlo simulation[13].

Any catalytic process deals with breaking and forming a new chemical bond and only specific types of reactant and catalyst can make this transition. In this thesis, reactants are the molecules in a gas phase that can make a bond with the surface. The active sites for adsorbing reactants on the surface are called adsorbents. Once the reactants become adsorbed on the adsorbents, they become adsorbates. Another important feature of a crystal that is influenced by its shape is catalysis. Catalysis is basically active sites of reaction that increase the reactivity of the surface. Typically some sites on the surface may react faster with the reactant than other sites. 
When Langmuir formulated the chemisorption model on a metallic surface he assumed there were two types of sites on a flat surface[14]. One energetically identical and one with no interaction. He wrote in his note "Most finely divided catalysts must have structures of great complexity[15]. In order to simplify our theoretical consideration of reactions at surfaces, let us confine our attention to reactions on plane surfaces. If the principles, in this case, are well understood, it should then be possible to extend the theory to the case of porous bodies. In general, we should look upon the surface as consisting of a checkerboard." Thus, he developed the first theory of heterogeneous catalytic surface. This characteristic and its consequences became further highlighted by Taylor; he wrote: "There will be all extremes between the case in which all atoms in the surface are active and that in which relatively few are so active." [16]

In this thesis, we use the Kinetic Monte Carlo method (chapter 3) and homogenization theory (chapter 2), to calculate the effective activity of patterned surfaces. In each surface that we study there are two types of interactive sites that are either distributed in a certain way or randomly. In reality, there are many different types of active sites. The key point of these sites is their availability for chemisorption from a liquid phase. Some of these cases can increase or decrease specific activity dramatically by increasing or decreasing the crystallite sizes. For example, in the metallic case, some metals such as gold and platinum can strongly hold and activate the reactants even in their smallest sizes, but not so strongly that they break the product. [17]. One of the mathematical models that we applied in the crystallization process with the catalytic surface was homogenization theory due to the diffusion of the molecules near the nonuniform surface, which comes from the modification of the continuum diffusion equation. The numerical method that we applied was the Mont Carlo simulation method since the process involves the complex stochastic behavior of many chemical species at a micro-level[18]. We use a standard kinetic Monte Carlo method to model the performance of the catalytic surface and then extend this to the simulating polymer chain, near and adsorbed on the surface[19].

Technically, when we model such a system in continuum limit, this will lead to considering nonuniform time evolution of the crystal growth. Under diffusion limited conditions, the continuum theory predicts that growth will follow at $t^{1 / 2}$ (interval time) law, whereas in general the evolution of the process depends on the rate of nucleation and growth of particular facets[13],[20]. 
A method for controlling crystal growth, that we consider in this thesis is polymer adsorption on a surface. In many biocompatible and chemical surfaces, it is possible to control the crystalline structure by polymeric growth modifiers. Different polymer adsorbates such as homopolymer chains, diblock copolymer chains, polymer brushes or polymer stars can be used to influence a crystal to grow in a certain direction by minimizing their free energies[21],[22]. Depending on the strength of the polymer chain and the surface energy, they can be adsorbed on a surface in such a way that they act as an insulator and cover the facets from growing and help other facets to grow faster[23].

However, in the polymer adsorption process we need the relation between the free energy difference and the functional phase space, since this relation only supports a canonical ensemble, and thus we applied the extended ensemble method in order to find the free energy difference for a continuous range of temperature.

\subsection{Thesis outline}

In this thesis, we want to study how to control the crystal structure and alter the rate of growth by simulation methods. Therefore, first we need to study the physical characteristic of the surface in order to understand the interactions of the adsorbing particles near the surface at a micro level. In order to grow a crystal surface with a specific pattern we need a substrate with different surface energies based on that pattern. In order to design such a crystal surface, we apply absorbed polymer to control and alter the rate of reactivity of the surface. In this thesis, we first study the effective reaction rate of patterned surfaces and then we consider the influence of adsorption of the polymer on those patterns for controlling the reaction event near the surface in order to create a surface structure with different surface energies. In order to study the effective rate of catalysis near a surface we developed a mathematical method to understand the physical properties of sites such as their size effect, orientation effect, and their coverages.

In Chapter 2 we explain the mathematical model that we developed for heterogeneous catalytic surface[24]. We will first start using the kinetic gas theory and examine 
the adsorption and diffusion event in a continuum model, and then by applying homogenization theory we explore the limits of the continuum theory for a heterogeneous catalytic system.

In Chapter 3 we apply the kinetic theory of gases and simulate the growth of the heterogeneous catalytic surface. We show that the kinetic Monte Carlo method is the best approach for simulating such a system [3]. Chapter 4 is the background knowledge of what we need for applying polymer adsorption on the surface. In order to study the adsorption of polymer onto a patterned surface, we need to consider different types of interaction from long range to short range interactions. In this chapter, we will talk about the tools and concepts that we need in order to simulate polymer adsorption onto a heterogeneous, attractive surface.

In Chapter 5 we develop a kinetic Monte Carlo method for adsorption of homopolymer chains onto the heterogeneous active surface. In this chapter we show the size effect and the importance of pattern on polymer adsorption.

In Chapter 6 we apply different copolymer chains onto the heterogeneous surface in order to understand the importance of orientation of monomers in a copolymer chain on crystal growth. Designing the best orientation of monomers in a copolymer chain will help us to achieve the desirable crystal surface. Since aiming at this goal is experimentally expensive, we can simulate such a system. The Kinetic Monte Carlo method can help us to find the best copolymer structure for crystal growth. 


\section{Chapter 2}

\section{Theory of Heterogeneous Catalytic Surface}

\section{$2.1 \quad$ Introduction}

Applying a catalyst in chemical processes can increase the reaction rate without being consumed during the reaction (see Figure 2.1)[25]. A catalyst is a substance that alters the internal energy of the chemical reaction by changing the thermodynamic path without disturbing the initial energy and final energy of the process. The catalyst can be liquid, gas or solid and its performance on a solid state will depend on its shape and size because of the activity of different facets a particular crystal presents. Figure 2.2 shows that reactant A becomes adsorbed on catalytic site forming a new chemical bond, and the product $\mathrm{C}$ will become desorbed after the chemical reaction is completed.

$$
\mathrm{A}+\text { Surface } \rightarrow \mathrm{C}
$$

There are two types of catalytic processes, heterogeneous and homogeneous catalysis. If the catalyst and reactants or their solutions are in the same phase it is called a homogeneous catalyst reaction; when they are in different phases it is called heterogeneous catalysis. From the point of view of applications, heterogeneous catalysis is more important than the homogeneous one. In most industrial projects they apply the heterogeneous process to speed up the rate of the interactions. 


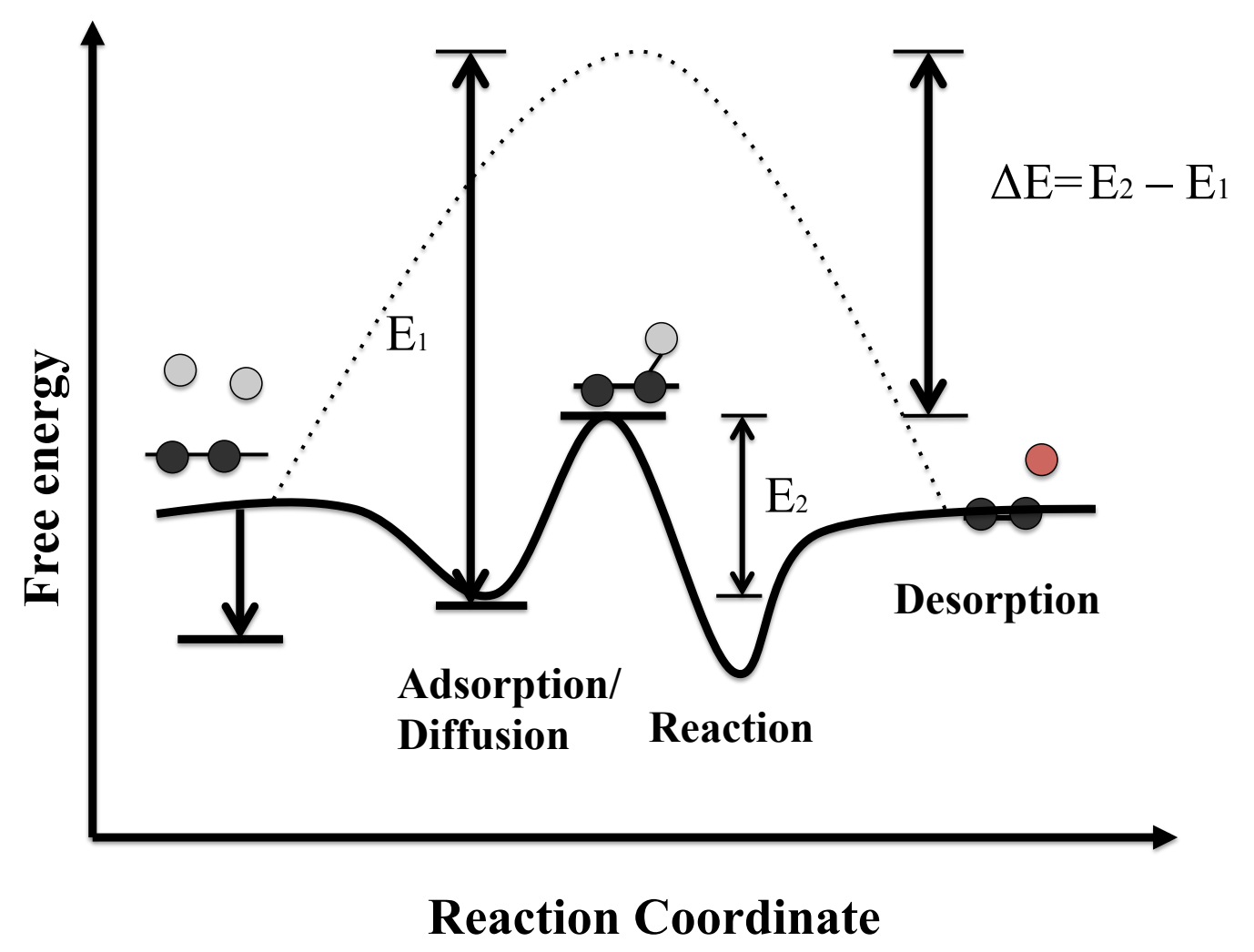

FiguRE 2.1: This figure shows the role of the catalyst during a chemical reaction. The catalyst is an additive material and does not need to be in a chemical reaction, however, it can increase the rate of reaction significantly. From left to the right shows the steps of the process, from collision to producing a product. $E_{1}$ is the energy required for this chemical process without a catalyst. $E_{2}$ is the energy required for making chemical bond with the surface by the presence of catalyst.

Many catalysts consist of precious metals, and therefore applying them in bulk in a chemical process is not economically efficient. Dispersing them in nanostructured form reduces the cost of applying them by maximizing the surface area exposure for reactions. As a result, by using nanostructured heterogeneous catalysts we can dramatically increase the performance by weight of this precious material[26]. In addition, some metals like gold, which is largely inert in bulk form, can exhibit strong catalytic activity in nanostructured form[27]. In particular, investigating the difference between bulk active metals and highly dispersed nanoparticulate form is something that needs to be studied more.

The phenomenon of applying material as a catalyst was first discovered by Berzelius in 1835 [28]. At the same time, Mitscherlich was also studying catalytic reactions by solids, introducing surface-reactants and contact- catalysis at that time. In 1895, Ostwald used catalysts in chemical reactions and called them an accelerator of reaction. His 


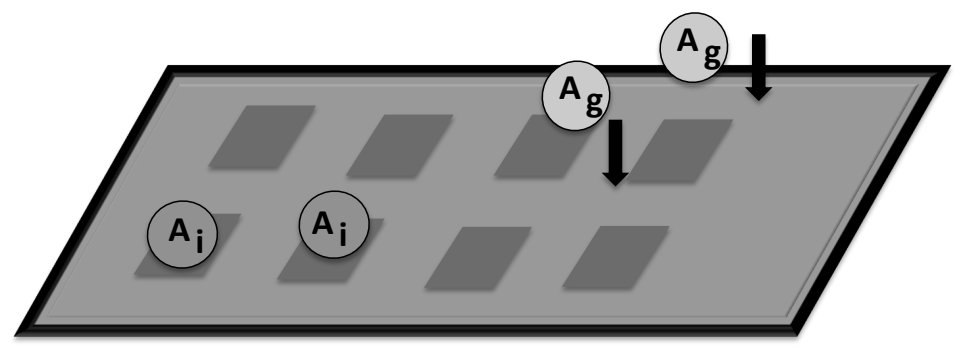

(a)

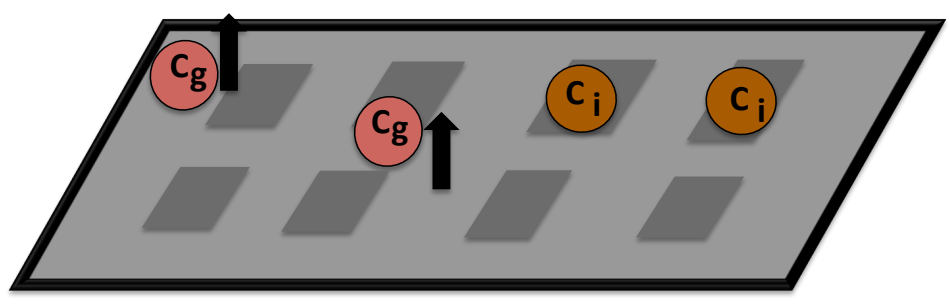

(b)

Figure 2.2: This figure shows all the phases of a chemical process when reactant gas $A_{g}$ converts to particle $C_{g}$. When reactant $A_{g}$ becomes stable and continues to make a bond with the molecules on the surface, it becomes $A_{i}$. Once the chemical bond between surface and $A_{i}$ created, the new particle $C_{i}$ is made. After a while, a new particle becomes free to go and leave the surface.

discovery was recognized with the Nobel prize for chemistry in 1909[15]. From that time, other scientists investigated different materials and found new types of active materials. Around 1913 Louis Jacques and Pierre Dulong discovered the reactivity of some metals such as iron, copper, silver, gold and platinum[29]. Later on Taylor et al. discovered that the reactivity of platinum is increased in the presence of a metal base. In the 1980s, a group reported the activity of clusters on titanum with the appearance of nonmetallic properties. This is one of the first studies where the size effect of gold sites has been pointed out[30]. The interplay of diffusion theory and reaction rate process is one of the interesting problems in statistical physics of disordered systems. Although tracking a single particle in random media has been discussed in many kinds of literature, examining spatial-active patterns is a subject that needs to be studied more[31].

In this thesis, we focus on the dynamics of a catalytic surface at the intermolecular 
level and cover the gap between the microscopic elementary reaction and the macroscopic performance using the homogenization approach. Our goal is first to understand nanostructured catalytic sites for reaction and second to design new patterns of heterogeneous catalytic surfaces in order to increase their efficiencies and reactivities[32]. We apply numerical method to understand such a dynamically nonuniform environment due to the different rates of interactions[33]. In this chapter we only use homogenization theory. In the next chapter we find the kinetic information of the system such as effective reaction rate and coverages of a surface by the Kinetic Monte Carlo method $(\mathrm{KMC})[34]$.

In this chapter, we consider two-dimensional surfaces, with different active sites using lattice with stochastic particle dynamics. Previous work has studied the pairwise reaction of particles upon meeting, including heterogeneous disordered surfaces and chemical reactions of dust grains in interstellar clouds[35],[36]. A key quantity which describes the performance of such systems is their steady-state efficiencies. In this thesis, we consider a binary system consisting of two types of binding energies and one type of molecule in the solution. The active sites are labeled as strong and weak (or zero) binding energy sites. The efficiency of a surface can be calculated by the Kinetic Monte Carlo simulation method which we will study this in the next chapter[37],[38]. We extended our analysis from a binary case near the surface to continuous distribution in the three-dimensional simulation box, where we include the gas (reactant) as well. In order to study the effective reaction rate near the binary surface, we applied the homogenization theory. Studying the behavior of heterogeneous influx near the surface and homogeneous Brownian motion far from the surface are only feasible through the homogenization approach.

\subsection{Catalyst Application}

One of the most common daily uses of catalysts is catalytic converters in automobiles, where their role is to reduce the exhaust of harmful gases due to the combustion of the fuel in an engine[39]. Also, a catalyst can be applied in the industrial production of ammonia, nitric and sulfuric acids and similar substances. Another example of applying catalysts is in manufacturing polymers. This was discovered in the 18th century the resin catalyst could participate in producing polyethylene. Catalyst also can exist in 


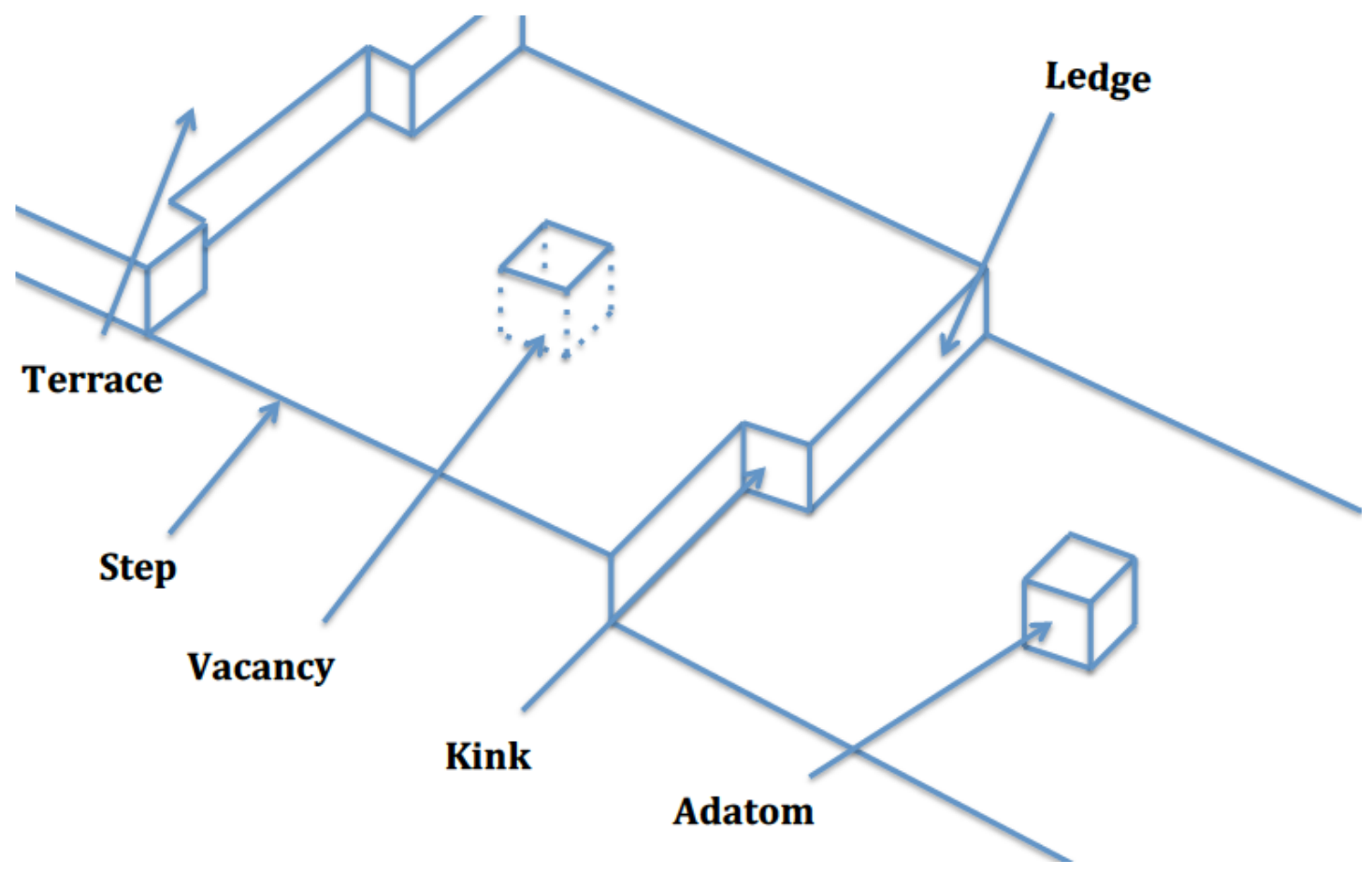

FiguRE 2.3: An illustration of different types of defect on nonuniform surfaces.

the spontaneous natural process[40]. Natural examples of catalyst are enzymes; they are a special type of protein with performance strongly related to their shapes. Another spontaneous catalytic process is the production of indigo dye by a mercury catalyst, a big catalyst discovery in 1897[41]. Another example is activated carbon, which has a specific number of adsorption sites so that molecules like hydrogen or nitrogen play reactant role with the carbon. Activated carbon has a microcrystalline structure that determines the activity of the surface area, the less ordered structure the more active it is. The adsorption isotherm represents the equilibrium states of such a system described by the Langmuir model[42], which is the fundamental concept in this chapter. Each microcrystalline structure can be considered as an active site with the periodicity of $\mathrm{L}$ from another microcrystalline in the Langmuir model. The arrangement of these layers will determine the activity of the surface area. In this Chapter, we will explain the Langmuir model and the importance of the physical structure of the surface.

\subsection{Theoretical Aspects of Catalytic Surfaces}

Several physical characteristics are important in this method, such as the mean free path of diffusive particles, relaxation time, diffusion rate, reaction rates, etc.[43]. When 
the interaction between the adsorbate and the surface is the Van der Waals type, the adsorbate molecules will diffuse uniformly and therefore their motions are Brownian. However, if the surface is chemically active for a chemisorption process, and the rate of diffusion of the particles and their relaxation time on the surface are not homogeneous, then they may not obey continuum diffusion equation anymore[44]. It turns out that the interaction properties of the surface depends on its internal structure, which can affect these physical characteristics (see Figure 2.3). Some factors such as the type of unit cell of the surface (e.g. bcc, fcc ,..) or defects of non-uniform surfaces (e.g. kink, step, terrace,...) can have a significant impact on adsorption and reaction rate near the surfaces[45]. Moreover, employing precious material under certain temperatures and pressures can alter the rate of interactions near the surfaces[46].

\subsubsection{Physisorption}

The first step in catalyst reaction is activating the reactant molecules by adsorption onto a catalyst surface. The bond between adsorbent and adsorbate determines the activation energy of a reaction[47]. The most common way of adsorption is physisorption, which molecules are bonded by Van der Waals forces to the surface. Due to the weak interaction of reactants with an adsorbent, this process does not disturb the structure or texture of the substrate and it is such that it can be reversed. For this reason, two or more than two layers of adsorbed molecules can be involved in the physisorption event. Any kind of molecule can interact with any solid surface through the Van der Waals forces. Although they are not effective as a catalyst, they apply as promoter to facilitate the adsorption of molecules onto a solid surface. These kinds of interactions are known as physisorption[48].

\subsubsection{Chemisorption}

In the chemisorption processes, the bond between reactants and surface lies between ionic and complete covalent[49]. Since absorbing to the catalyst surface with a chemical bond makes a new substance, a chemisorption is a unique event. It means that only certain adsorbent-adsorbate combinations can be formed. 
Chemisorption only happens in a single or a monolayer of adsorbed molecules. Moreover, if one site occupied by adsorbed molecule no additional particle can occupy on this site for chemisorption.

The adsorption of molecules onto a surface is a general phenomenon of any surface interaction process. In the case of a catalytic surface it is feasible to break down the continuous chemical process into the following steps:

1. Diffusion on the surfaces in the gas phase

2. Adsorption of one or more reactants onto the catalyst sites

3. Reaction of reactants with active sites

4. Desorption of products from the surface

5. Diffusion away from the surface

\subsubsection{Dynamics of the Gas}

After physical properties of the system, another important factor that determines the evolution of the system is the dynamics of the gas near the surface. This depends on many factors, such as mean free path, mean free time and collision rate. These quantities are related to each other, as described by the kinetic theory of gases for reactants in the gas phase[50]. For simplicity, we assume a single species of gas to be like hard spheres, meaning that there is no interaction between them. This is because in the gas phase the mean free path of the reactant has the maximum length.

\subsubsection{Mean free time}

The mean free time is the average interval time between two subsequent collisions. If a particle has its first collision at $t=0$, then the probability of no more collisions happening at $t=0$ is $P_{\text {time }}=1$. In classical gas kinetic theory, we assume that multiple collisions never happen for a molecule[51],[52]. We define a contact frequency $\nu$ to find the probability that the particle does not experience any contact at time interval $t$ to 
$t+d t$. Let $\nu d t$ denote the probability that a given molecule experiences a collision between $t$ and $t+d t$. Then the probability of no collision up to time $t+d t$ is given by:

$$
\mathrm{P}_{\text {time }}(t+d t)=\mathrm{P}_{\text {time }}(t)(1-\nu d t) .
$$

This linear equation can be converted to give a differential equation for $\mathrm{P}_{\text {time: }}$ :

$$
\frac{\mathrm{dP}_{\text {time }}}{d t}=-\nu \mathrm{P}_{\text {time }}
$$

Which has a solution:

$$
\mathrm{P}_{\text {time }}(t)=e^{-\nu t}
$$

In order to find a complete map of the distribution of free time of a given molecule, we define a distribution function $\mathrm{P}_{\text {time }}$.

$$
\tau=\int_{0}^{\infty} \mathrm{dtf}_{\text {time }}=\int_{0}^{\infty} \mathrm{dt} \operatorname{tP}_{\text {time }}(t)=\int_{0}^{\infty} \mathrm{dt} \mathrm{t} \nu e^{-\nu t}=\frac{1}{\nu}
$$

where $\tau$ is the average time between collisions.

\subsubsection{Mean free path}

The mean free path is the average distance traveled by a molecule between two subsequent collisions. The free path distribution equation of a molecule can be derived in the same way of mean free time by substituting $P_{\text {path }}$ as a probability of traveling a distance given by[53]:

$$
\lambda=\int_{0}^{\infty} \mathrm{dssP}_{\text {path }}(s)=\int_{0}^{\infty} \mathrm{ds} \mathrm{s} \alpha e^{-\alpha s}=\frac{1}{\alpha}
$$

the probability of a collision occurring in the elementary distance $d s$ which is proportional to $s$ and we write this as a distance $\alpha d s$ that next collision is happening. Now we obtain the average distance and average time between two collisions, and therefore, the ratio of the two equations (2.6) and (2.5) will represent the average speed of a molecule in a system.

$$
\frac{\lambda}{\tau}=v
$$




\subsubsection{Collision rate}

A collision event between two molecules happens in many chemical and physical processes. For a reaction in the gas phase and a substrate, the collision between reactant molecules and the substrate is important in the dynamics of the problem.[54]. According to the gas kinetic theory, the average molecular speed in terms of gas temperature and molecular mass is given by $\bar{v}=\sqrt{\frac{8 k_{B} T}{\pi m}}$

$$
Q=A \sqrt{\frac{8 k_{B} T}{\pi m}} \rho
$$

Where $\rho$ is the density of molecules per volume, $k_{B}$ is the Boltzmann constant, $T$ is the temperature, $m$ is the mass of the particle. and the collision frequency is simply given by:

$$
Q=A \bar{v} \frac{N}{V}
$$

where $\mathrm{A}$ is cross-section for scattering of the particles. $N$ is the number of molecules which lie within radial distance $d$ of the path $\bar{v} t$ before the collision occurs and $\mathrm{V}$ is the volume swept-out. In what follows, we consider molecules as hard spheres with no long range interactions. Since their collisions happen in a volume of space we consider a tubular volume in which particles collide with each other in the cross section of this tube (see Figure. 2.4)[55].

$$
\text { Volumn swept-out }=\pi d^{2} \bar{v} t
$$

\subsubsection{Diffusion}

The collision between molecules makes their motion stochastic. The kinetic theory of gasses developed by Maxwell can describe this motion, where diffusion events result from the collision of molecules in a liquid or gas[56]. Brownian motion is one of the mathematical models that refers to the random motion of the molecules in a solution. Molecules in liquid or gas have different speeds and move in different directions and this makes their diffusion pattern completely random. The simplest model of Brownian motion is the Wiener process which is a stochastic Markov process where the values of the system change in an uncertain way[57]. The Markov process itself refers to the 


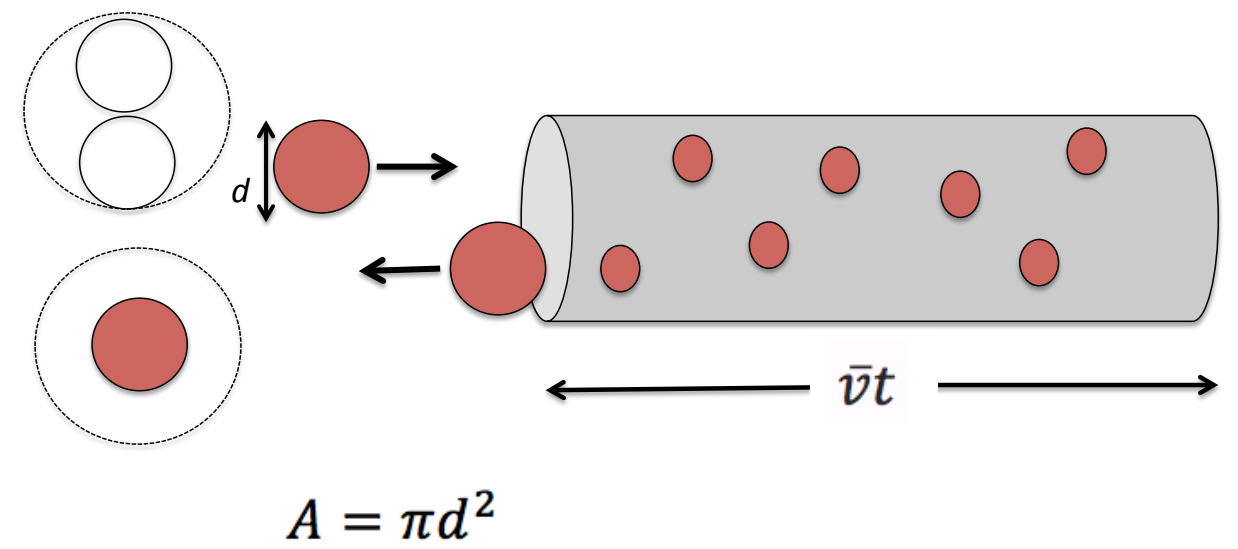

Figure 2.4: The collision of moving molecule with other random molecules. The volume amount of a tube in which particles collide with each other in the cross section of this tube. A is the cross-section for the scattering of the particle and $d$ is the radial distance of the path. $\bar{v} t$ is the path distance before the collision occurs.

likelihood of each state only depending on the next immediate point of time. Therefore, transition from one state to another after time interval $s$ can be defined as:

$$
P\left(W_{t+s} \in d y \mid W_{s}=x\right) \equiv p(s, x ; t, y) d y=\frac{1}{\sqrt{2 \pi(t-s)}} \exp \left(\frac{-(y-x)^{2}}{2(t-s)}\right) d y
$$

where $x$ is the place of one particular molecule at time $t=s$ and $y$ is the conditional place of the molecule at time $t=s+d t$, where the molecule has been already in $x$. where $p(s, x ; t, y)$ is called the Gauss kernel function. The Markov process $X=\{X t, t \geq 0\}$ is a diffusion process if the following limits exist:

$$
\left\{\begin{array}{l}
\lim _{t \downarrow s} \frac{1}{t-s}=0 \\
\lim _{t \downarrow s} \frac{1}{t-s} \int_{|y-x|>\epsilon}(y-x) p(s, x ; t, y) d y=\alpha(s, x) \\
\lim _{t \downarrow s} \frac{1}{t-s} \int_{|y-x|>\epsilon}(y-x)^{2} p(s, x ; t, y) d y=\beta^{2}(s, x)
\end{array}\right.
$$


where $\alpha$ is a drift coefficient (at time s and position $\mathrm{x}$ ) and $\beta$ is the diffusion coefficient (at time s and position $\mathrm{x}$ ). If $X=\{X t, t \geq 0\}$ is a diffusion process, then the transition density $p(s, x ; t, y)$ is given by:

$$
\frac{\partial p(x, y)}{\partial t}+\frac{\partial}{\partial y}\{\alpha(t, y) p\}-\frac{\partial^{2}}{\partial y^{2}}\left\{\beta^{2}(t, y) p\right\}
$$

The standard Wiener process is a diffusion process with drift $\alpha(s, x)=0$ and diffusion coefficient $\beta(s, x)=1$. In continuum theory, this is known as heat equation and is one the three fundamental solutions of Partial Differential Equation (PDEs).

$$
\frac{\partial p(x, y)}{\partial t}=\frac{1}{2} \frac{\partial^{2} p(x, y)}{\partial^{2} x}
$$

We will explain the numerical aspects of this model in the Numerical method section.

\subsubsection{Reaction Mechanism}

Collision rate and adsorption rate are the factors that describe the reaction rate near the surface. These key parameters are related to the reaction mechanism near the surface. In order to study the rate of interactions near the surface, we need to examine the speed of different chemical processes near the surface in the equilibrium state. The most common example of the description of a reaction mechanism near the surface is the Langmuir isotherm. Although this theory is based on the assumption that the reactivities of the active sites are similar for different surface coverage, in this thesis, we prove that this assumption may not be true for nanostructured active sites. In lower coverage and more distributed pattern active sites, the reaction rate is slightly higher than the compact format. However, this theory will help us to understand the adsorption isotherm near the surface. In this study, we will show that adsorption will be nonuniform with some active sites with higher adsorption strength covered first by reactants. 


\subsubsection{Langmuir Isotherm}

The Langmuir adsorption model will help to quantify the number of adsorbates as a function of pressure[58].

$$
A+M \underset{k_{d}}{\stackrel{k_{a}}{\rightleftharpoons}} A M
$$

Where $k_{a}$ is a rate constant for adsorption and $k_{d}$ is a rate constant for desorption. This theory which is also referred to as a Langmuir isotherm includes several assumptions [59],[60]:

- A particular solid surface has a fixed number of identical adsorption sites, each of which assigns how many reactants can be occupied by one molecule.

- Reactants behave identically on a surface.

- Only one monolayer will form on a substrate in order to react with the surface.

- There is no adsorbate-adsorbate interaction after adhesion.

In this model, the rate of adsorption depends on the number of active sites that cover the surface and the frequency of the contact of the reactant with the surface. From the above assumption if one site is already occupied it is not available for another reactant. As a result, only available sites have the potential to be a target for a reactant[61]:

$$
r_{a} \propto Q P_{a} N(1-\theta)
$$

where $r_{a}$ is the rate of adsorption, $\mathrm{Q}$ is the collision rate of reactant per unit area, $P_{a}$ is the probability of adsorption and $\mathrm{N}$ is the total number of reactants in the system. $\theta$ is given by:

$$
\theta=\frac{\text { Number of Occupied Adsorption Sites }}{\text { Number of Available Adsorption Sites }}
$$

Since the rate of adsorption and desorption depends on the intrinsic properties of active sites, by multiplying rate constant to Eq. 2.16,

$$
r_{a}=k_{a} Q P_{a} N(1-\theta)
$$

Similarly, the rate of desorption is given by:

$$
r_{d}=k_{d} P_{d} N \theta
$$


In equilibrium the rate of adsorption will be equal to the rate of desorption:

$$
k_{a} Q P_{a} N(1-\theta)=k_{d} P_{d} N \theta,
$$

We assume all the particles that are adsorbed on one active site can potentially perform chemical interaction with the surface, so $P_{a}=P_{d}=1$. Thus the Eq. 2.20 will become:

$$
k_{a} Q N(1-\theta)=k_{d} N \theta
$$

If we define a new constant $k$, which is the ratio of $\frac{k_{a}}{k_{d}}$, then:

$$
\begin{aligned}
& \frac{1-\theta}{\theta}=\frac{1}{k Q} \\
& \theta=\frac{k Q}{1+k Q}
\end{aligned}
$$

Since the collision rate and pressure of the gas are in a direct relationship, at very low pressure $k Q<<1$ and $\theta=k Q$, whereas at high pressure $k Q>>1$ and $\theta=1$. As a consequence, the plot of coverage versus pressure should look like Figure. 2.5.

\subsubsection{Homogenization Theory}

Finding the macroscopic properties of a system by averaging the microscopic details of the system can be achieved by a technique known as homogenization. This illustrates the known example of when we consider a medium made up of two types of material: one has high conductivity and the other low conductivity. Technically the total conductivity of this system will be driven by the low conductance and it is not simply the average of high and low conductivities, so the effective conductivity is given by modification of the low conductivity by considering high conductivity in the system and is different from the mean of the two conductivities. Homogenization is a mathematical tool that leads to describing a system by macroscopic characterization with additional parameters or multiplication. This mathematical tool can be applied in[62]:

- systems with variation of boundary conditions

- systems with different rates of diffusion; 


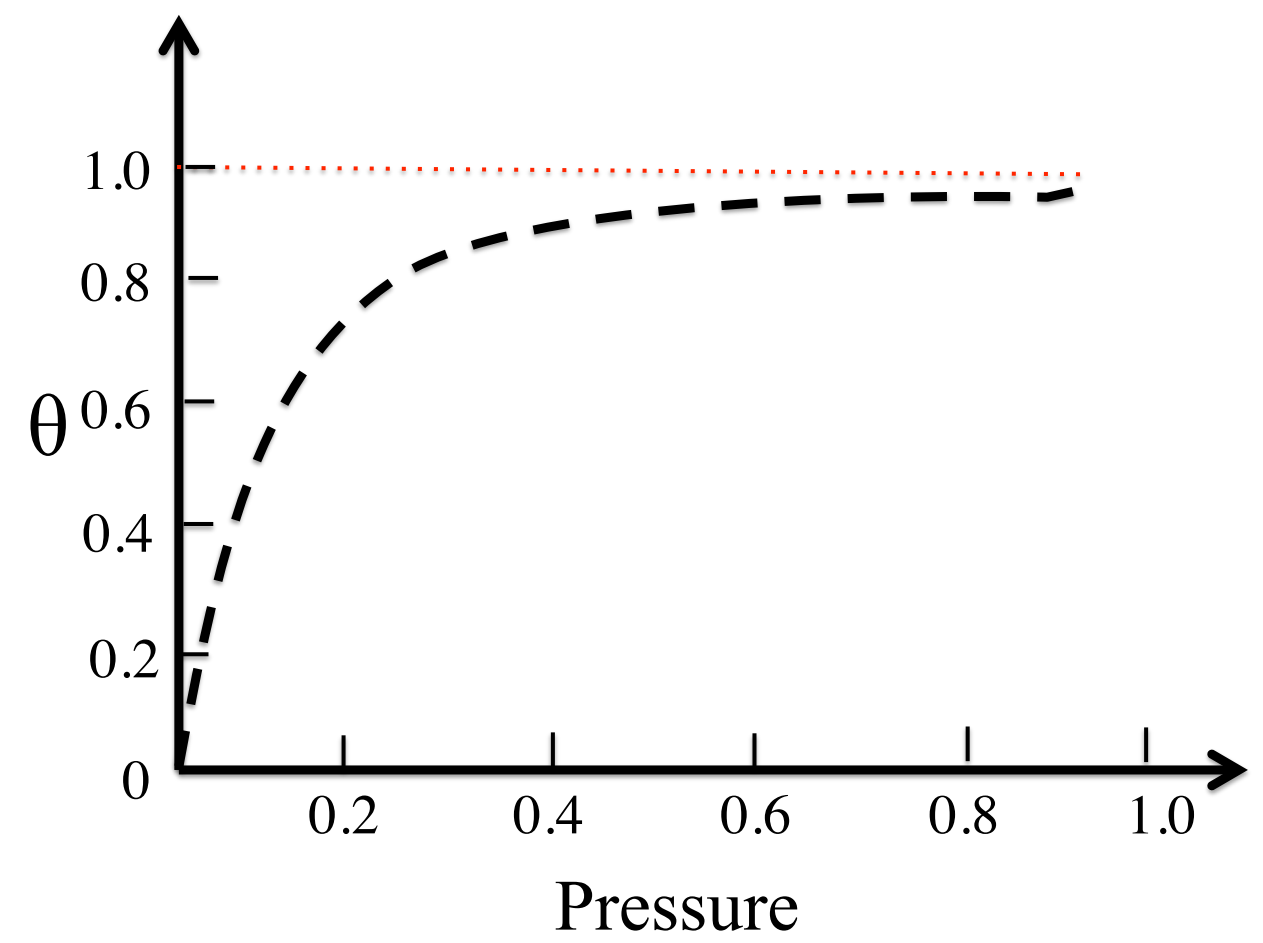

Figure 2.5: This figure demonstrates coverage versus Pressure. As the pressure increases the coverage will increase up to saturation level. Coverage has a direct relationship with the rate of interaction as well. The pressure varies as does the surface coverage.

- systems with variation of oscillations etc.

Several kinds of literature have applied this method to their work. Lund and Hendy used homogenization theory to calculate effective slip boundary condition for flow over heterogeneous surfaces[63],[64]. Also a group Arriaga et al. applied this method in photonic crystals[65]. Chiang et al[66]. applied this mathematical method in different media such as seepage in rigid porous media, heterogeneous elastic materials, deformable porous media, and wave propagation in inhomogeneous media. They extended the mathematical technique of homogenization to the flow of fluid and heat through a porous medium to explain the asymptotic behavior of fluid flow in inhomogeneous media.

\subsubsection{Diffusion toward Heterogeneous Surface}

We consider a problem of diffusion of the reactants over the periodic heterogeneous surface with a different rate of reactivities. The key feature of this heterogeneous surface 
is the life expansion of the adsorbed reactant over the surface[67]. The mathematical description of such a phenomenon involves a differential equation of diffusion of particles that diffuse in space at the scale of the medium. If we rewrite Eq. 2.14 based on diffusion equation of particles onto the $\mathrm{x}-\mathrm{y}$ plane, then in the polar coordinate:

$$
\frac{\partial \rho(r, t)}{\partial t}=D \frac{\partial^{2} \rho(r, t)}{\partial r^{2}}
$$

In this thesis we consider non-interacting particles, then in a closed system the total number of particle will become constant and (Eq. 2.14) leads to:

$$
\int_{-\infty}^{+\infty} \rho(r, t) d r=1
$$

For a uniform diffusive system in steady state we expect:

$$
\frac{\partial}{\partial x_{i}}\left[D \frac{\partial \rho}{\partial x_{j}}\right]=0
$$

Where $\mathrm{D}$ in uniform media is constant and $x_{j}$ is one arbitrary direction. 


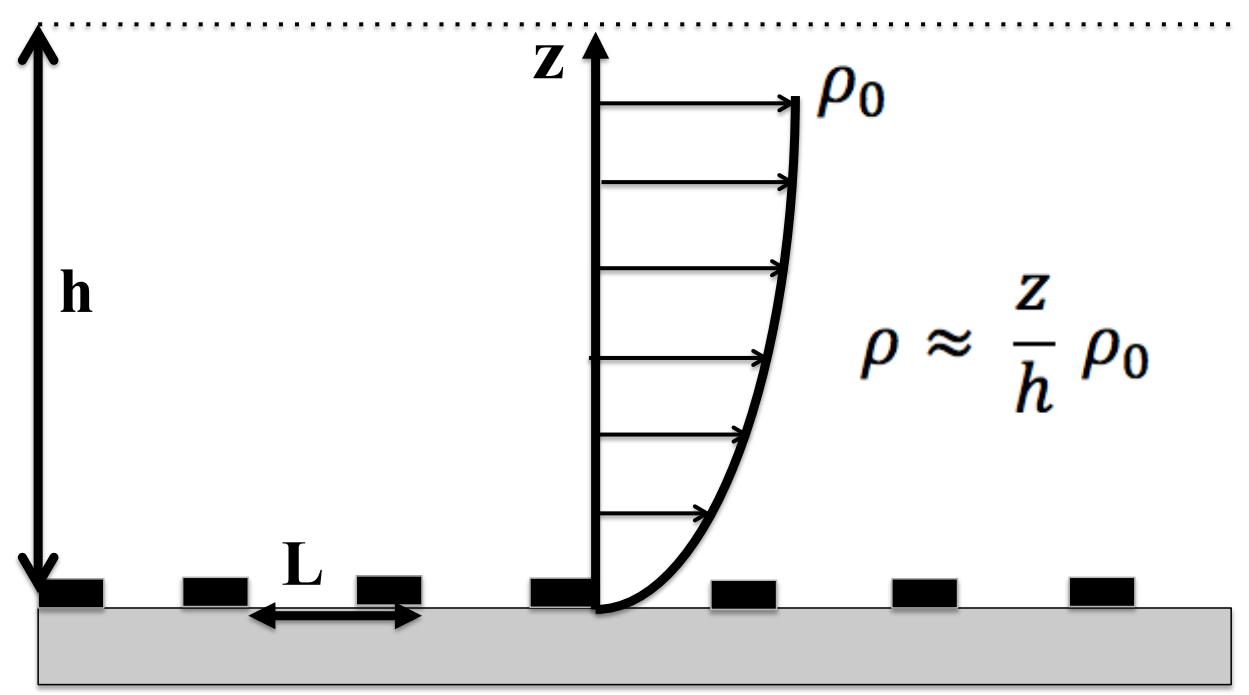

FiguRE 2.6: Density distribution along the z-axis. Due to the heterogeneity of the surface, the distribution of density that changes linearly as a fraction of the height times the normal density on a flat surface, it approximately changes with this ratio for a heterogeneous surface. This exhibits the presence of additional term that could approximately estimate the distribution of the density over a heterogeneous surface.

Figure. 2.6 shows the diffusion of reactant near a heterogeneous surface with the periodicity of L of active sites. For ideal gas near a neutral surface, we expect density of the gas distributes linearly correspond to the height of diffusion and initial density of the gas. Whereas in heterogeneous surface this distribution does not completely correspond to the equation that is written in the Figure. 2.6 and the effective reactivity of the active sites must be added as a coefficient to the equation. The problem will become more specific by considering the dynamics of the particle near the surface which in steady state the rate of a collision near the surface and diffusion of the system are identical:

$$
D \frac{\partial \rho}{\partial x}=k_{1} Q
$$

where left side of equation refers to the adsorption rate of Langmuir-adsorption model and $\mathrm{Q}$ is the collision rate $\left(Q=\left(\sqrt{\frac{8 k_{B} T}{\pi m}}\right) \rho\right), D \sim \bar{v} \lambda$ and $l=\lambda / k_{1}$ which comes 


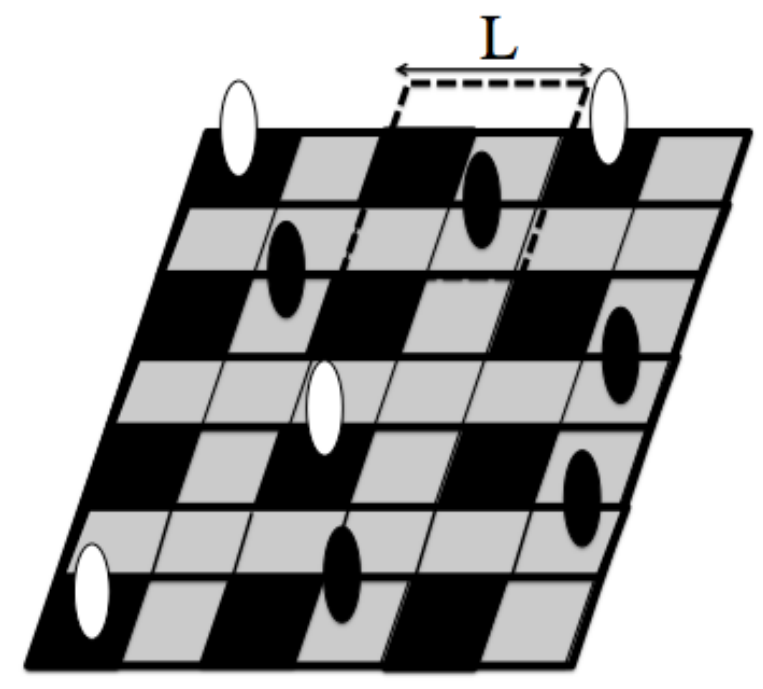

Figure 2.7: (An illustration of a catalyst surface, showing active sites (black) and non-active sites (grey) distributed in a pattern of period $L$ with adsorbed particles (white) and non-absorbed particles (black).

from Eq. 2.8, Eq. 2.14 and Eq. 2.7. We expect this equation to be valid in continuum limits; however, when we test it away from the continuum theory the homogenization theory will not be valid anymore. In order to tackle this problem, we need to consider the heterogeneous boundary condition. Diffusion limited condition or controlled diffusion is when the diffusion of reactant is rare. In a solution when the number of reactant gas is low the number of reaction becomes more limited due to the less number of diffused particles.

\subsection{Homogenization of Heterogeneous Catalytic Surface}

The catalyst surface consists of a patterned array of active sites, over which the activity can vary periodically with period $L$ Figure. 2.7. We will also assume the system has reached steady-state and is operating under diffusion-limited conditions. We also make a number of assumptions to simplify the mathematics. Once the reactants have adsorbed to the surface, we assume that they cannot diffuse on the surface before the catalytic reaction take place. In principle, desorption could occur, but in this work we will assume that the desorption rate is negligible. Likewise, we assume that the catalytic conversion process is also irreversible. These assumptions allow us to simplify the mathematical problem so that results from homogenization theory can be applied, although this comes at the expense of limiting the applicability of the model. For 
instance, we do not consider the possibility that the catalytically active sites are not the most favorable sites for adsorption. We will consider a binary pattern on the surface, with low activity sites and high activity sites distributed periodically over the surface. Each site can only be occupied by one reactant molecule at a given time (Fig. 2.7). We break the adsorption and catalytic processes down into four steps with associated rate constants $k_{1}, k_{2}, k_{3}$ and $k_{4}$ respectively:

1. Adsorption a gas particle of type $A_{g}$ collides with an active site $S_{i}$ and is adsorbed to this site with probability $k_{1}$ :

$$
A_{g}+S_{i} \rightarrow A_{i}
$$

2. The adsorbed particle desorbs from the surface to re-enter the gas phase without having undergone catalysis with rate $k_{2}$ :

$$
A_{i} \rightarrow A_{g}+S_{i}
$$

3. Adsorbed particles of type $\mathrm{A}$ are converted to particles of type $\mathrm{C}$ with rate $k_{3}$ :

$$
A_{i} \rightarrow C_{i}
$$

4. Particles of type $\mathrm{C}$ desorb from active sites with rate $k_{4}$ :

$$
C_{i} \rightarrow C_{g}+S_{i}
$$

In this thesis, once the product is made, we disregard them from the process since they do not have any interactions with other particles in the system. However, in some studies, these particles themselves may play a key rule on the ultimate result and need to be considered during the chemical process. To simplify the analysis, we will assume that step (4), the desorption of the reaction product $B_{\mathrm{i}}$, occurs much more rapidly than the reaction step (i.e. $k_{3} \ll k_{4}$ ) and that step (2), the desorption of the reactant is much slower than the reaction step (i.e. $k_{2} \ll k_{3}$ ). With these assumptions, the step (3) is the rate limiting reaction step at the surface. Under these assumptions, the Langmuir 
equation for the fractional surface coverage $\theta_{i}$ of the site $i$ is:

$$
\frac{d \theta_{i}}{d t}=k_{1}\left(1-\theta_{i}\right) Q-k_{3} \theta_{i}
$$

where $Q$ is collision rate per unit area over the entire surface. The first term is the rate of adsorption of particles that have diffused to the surface on an empty active site. The second term is the rate of catalytic conversion over the surface. Under diffusion-limited conditions, the fractional surface coverage $\theta_{i} \ll 1$, so the steady state coverage will be given by:

$$
\theta_{i}=\frac{k_{1}}{k_{3}} Q
$$

In order to proceed further, we need to introduce a model to describe the transport of the gas A to the catalyst surface. We use a Fickian diffusion equation, which describes the flux of gas A as it is consumed at the catalyst surface:

$$
\frac{\partial \rho_{A}(r, t)}{\partial t}=D \nabla^{2} \rho_{A}(r, t)
$$

where $D$ is the diffusion coefficient and $\rho_{A}$ is the density of the gas A. Under steady-state conditions the density of gas A satisfies(from 2.26):

$$
\nabla^{2} \rho_{A}=0
$$

Before steady state, the coverage of surface by absorbing molecules is a function of time and depends on the rate of adsorption and reaction of the reactants with the surface. On the steady state, the rate of adsorption becomes as the same as the rate of conversion, and the particle influx at the catalyst surface $\Gamma$ (with unit normal vector $\vec{n}$ ) is equal to the average density of converted particles per unit time (from 2.27):

$$
-\left.D \vec{n} \cdot \nabla \rho_{A}\right|_{\Gamma}=-\frac{d\left[C_{g}\right]}{d t}=\frac{k_{3} \theta_{i}}{a_{i}}
$$

where $a_{i}$ is the area unit of active site $i$, which we considered has to be 1 . where we have used the fact that $\frac{d\left[C_{g}\right]}{d t}$ is proportional to the second term on the right-hand side of (Eq. 2.28). By balancing the flux of gas $A_{g}$ into the site $i$ with the rate of adsorption of the gasses by mass, we can obtain a steady-state boundary condition for the gas density, 
$\rho_{A}$ over the surface:

$$
-\left.D \vec{n} \cdot \nabla \rho_{A}\right|_{\Gamma}=-\frac{k_{1} Q}{1+\frac{k_{1} Q a_{i}}{k_{3}}},
$$

where, according to gas kinetic theory, the collision rate is $Q=\left.\frac{1}{4} \sqrt{\frac{8 k_{B} T}{\pi m}} \rho_{A}\right|_{\Gamma}$, where $m$ is the mass and $T$ is the temperature of the gas A. Under adsorption-limited conditions $\left(k_{1} Q a_{i} \ll k_{3}\right)$ the flux is proportional to the density of the gas $\mathrm{A}$ near the surface:

$$
-\left.D \vec{n} \cdot \nabla \rho_{A}\right|_{\Gamma}=-\frac{k_{1}}{4} \sqrt{\frac{8 k_{B} T}{\pi m}} \rho_{A},
$$

which reduces to a simple mixed boundary condition for the density of gas A at the surface:

$$
-\left.l \vec{n} \cdot \nabla \rho_{A}\right|_{\Gamma}=\rho_{A},
$$

where the constant $l=\frac{D}{k_{1}} \sqrt{\frac{2 \pi m}{k_{B} T}}$ has the dimension of length and can be interpreted as the size of the region over which the gas $\mathrm{A}$ is depleted by adsorption and catalysis at the surface.

Gas kinetic theory tells us that $D \sim \lambda \bar{v}$ where $\bar{v} \sim \sqrt{\frac{k_{B} T}{m}}$ is the mean velocity of the gas molecules and $\lambda$ is the mean free path of the gas molecules. As a consequence, the length $l$ is found to scale as the ratio of mean free path to the probability of adsorption at an active site:

$$
l \sim \frac{\lambda}{k_{1}}
$$

The discussion above, in particular, the boundary condition (2.35), applies to a catalytic surface with a uniform distribution of active sites. However provided the distribution of active sites on the surface varies slowly enough, we would still expect (2.35) to hold, with $l \sim \lambda / k_{1}$ becoming a function of location on the surface. Conceptually we would regard the probability of absorption $k_{1}$ as varying across the surface on the length scale $L \gg \lambda$. Regions with high $k_{1}$ would represent a high concentration of active sites on the surface, while regions with low $k_{1}$ would represent a low concentration of active sites.

We are interested in determining the effective activity of a catalytic surface with a 
density of active sites that varies over the surface. By effective activity, we mean the activity of a homogeneous surface (i.e. a surface with a constant $k_{1}$ ) that would produce the same overall rate of conversion of gas $\mathrm{A}$ as the heterogeneous surface when viewed macroscopically. To compute the effective activity, we use homogenization theory $[68,69]$. In what follows, we assume that $l \sim \lambda / k_{1}$ varies periodically over the catalytic surface with period $L$. In this case we expect $\rho_{A} / \nabla \rho_{A} \sim L$ near the surface. Consequently, it is convenient to introduce a dimensionless set of spatial coordinates $x^{*}=x / L$. The scaled boundary condition at the surface then becomes:

$$
-\frac{l}{L} \vec{n} \cdot \nabla^{*} \rho_{A}(r, t)=\rho_{A},
$$

Thus the ratio of $L / l \sim(L / \lambda) k_{1}$ emerges as an important parameter in the effective performance of the catalyst.

By assuming (eq. 2.37) holds, two limiting cases can be examined. The first limit occurs when $l \ll L$, so that the boundary condition reduces to:

$$
\rho_{A}=O\left(\frac{l}{L}\right)
$$

which at zeroth order in $l / L$ would give a purely diffusion-limited reaction. However by calculating the first order correction in $l / L$ we can obtain an effective reactivity of the heterogeneous surface. The effective activity of the catalyst surface (say, the plane defined by $z=0$ ) is given by (see appendix A):

$$
k_{e f f}=\left\langle k_{1}(x, y)^{-1}\right\rangle^{-1}
$$

to first order in $l / L$, where the angle brackets indicate the average of the quantity $k_{1}(x, y)$ over the surface. Note that in this case the effective activity is given by the area-weighted harmonic mean of $k_{1}$ rather than the arithmetic mean. To examine the implications of (2.39), consider a surface composed of two types of region, with adsorption probabilities $k_{a}$ and $k_{c}$ respectively, that are periodically arranged over that surface. If a region of type $a$ occupies an area fraction of $\phi$ on the surface, then the effective activity is given by:

$$
k_{e f f}=\frac{k_{a} k_{c}}{\phi k_{c}+(1-\phi) k_{a}} .
$$


If the coverages of the sites are such that $k_{a} / \phi \gg k_{c} /(1-\phi)$ then this reduces to $k_{e f f} \sim k_{c} /(1-\phi)$, so if the two regions occupy similar area fractions on the surface, but $k_{a} \gg k_{c}$, the effective absorption probability scales with the smaller value $k_{c}$. This is a direct consequence of the harmonic mean appearing in (2.39).

The second limit we consider occurs when $L \ll l$. Now the first-order boundary condition becomes

$$
\vec{n} . \nabla \rho_{A}=O\left(\frac{L}{l}\right)
$$

In this case it can be shown by homogenization that the effective adsorption probability is given by:

$$
k_{e f f}=\langle k(x, y)\rangle
$$

where now instead of the harmonic mean we have the arithmetic mean of $k_{1}$.

Again it is instructive to consider a surface where reactants are more likely to adsorb in regions of type $a$ than regions of type $b$ (so $k_{a}>k_{c}$ ). If the coverage of the regions of type $a$ is $\phi$ then $k_{e f f}=\phi k_{a}+(1-\phi) k_{c}$. Again if $k_{a} \gg(1-\phi) k_{c} / \phi$ then $k_{e f f} \simeq \phi k_{a}$ so the effective absorption probability of the surface scales with the larger value $k_{a}$.

To summarize, homogenization theory predicts that the effective performance of a microstructured surface with adsorption probability Eqn. (2.34) will be that of a homogeneous surface with $k_{e f f}$ :

$$
-D \sqrt{\frac{2 \pi m}{k_{B} T}} \vec{n} \cdot \nabla \rho_{A}(r, t)=k_{e f f} \rho_{A},
$$

where $k_{e f f}=\left\langle k_{1}(x, y)^{-1}\right\rangle^{-1}$, the harmonic mean, when $L \gg l$ and $k_{e f f}=\langle k(x, y)\rangle$, the arithmetic mean, when $L \ll l$. Note that in both cases the effective adsorption probability will be independent of the particular pattern of active sites on the catalyst surface. In the next chapter, we will test these predictions using kinetic Monte Carlo simulations. We will also examine their applicability away from the continuum limit. 


\subsection{Conclusion}

Heterogeneous catalysis is of fundamental scientific interest in chemistry and materials science, as well as being of significant economic value to society[70, 71]. The effective performance of a particular catalytic system can depend on the interplay between reactant availability and the surface area of the heterogeneous catalyst. When there is a high concentration of reactants in the system, one would expect that the effective catalytic activity of a nanoparticulate catalyst by weight will increase with its surface area, suggesting that maximizing the surface to volume ratio of such a catalyst could reduce its cost without sacrificing performance [72-74]. This has stimulated a great deal activity in the search to fabricate high-surface area nanoparticulate metal catalysts[75]. However, nanostructured catalysts do have their disadvantages. The high surface area of a nanostructured catalyst may reduce its stability, which can be a drawback when operating at high temperatures[76]. Furthermore, under diffusion-limited conditions, reactants may not be able to access the entire surface of a nanostructured catalyst[77, 78] if the depletion zone of reactants about active sites on the catalyst start to overlap. Gas kinetic theory predicts that the size of this depletion zone will scale as the mean free path of the gas molecules[79], so one might expect that under diffusion-limited conditions the effective reactivity of a heterogeneous catalyst would depend on the structure of the catalyst at that scale.

In this chapter, we considered the problem of computing the effective performance of a catalyst under diffusion-limited conditions, given a particular spatial arrangement of catalytically active sites on the catalyst surface. Indeed, the surface of a typical heterogeneous catalyst possesses active sites with a range of activities distributed across a range of length scales. Under standard conditions, the mean free path of molecules in air is on the order of $100 \mathrm{~nm}$ so we might expect that nanostructured catalysts may behave differently under diffusion-limited conditions to catalysts that are structured on larger or smaller length scales. In our model, we considered a surface with a periodic array of active sites of period $L$. We describe the kinetics of the absorption of a reactant onto this catalytic surface using a Langmuir model, with the catalytic process described by a series of distinct elementary steps, including adsorption, reaction, and desorption. The theory developed here also applies to surfaces that are covered by small catalytically-active particles[80]. Again, one may regard such a support as a catalyst 
with a heterogeneous distribution of active "sites", where in this case each "site" represents a catalytically active nanoparticle. In this case, one may have a great deal of control over how the particles are distributed over the support (e.g. see [81]) or indeed the distribution may change over time due to Ostwald ripening [82] or other process associated with the catalytic reaction [83]. Our approach allows one to compute the effective catalytic activity of a support in terms of its coverage by catalytically active particles. We compute the effective activity of such surfaces under diffusion-limited conditions using two approaches. In this chapter, we used a continuum diffusion equation to describe the transport of reactants to the surface, and applied mathematical homogenization techniques $[84,85]$ to compute an effective activity in a limit where the pattern period $L$ is much less than the size of the depletion zone about the active sites, which is of order $\lambda$. In the next chapter, we test our model with the Kinetic Monte Carlo simulation method to explore the limitations of the heterogeneous catalytic surface in continuum model. 


\section{Chapter 3}

\section{Monte Carlo Simulation of the Effective Reactivity of a}

\section{Heterogeneous Catalytic Surface}

\subsection{Introduction}

In the previous chapter, we applied a continuum mathematical model in order to compute the effective reaction rate near a patterned surface. The effective performance of the catalysts under diffusion limited conditions was the subject that we mainly focused on. We applied gas kinetic theory to demonstrate the importance of the size of active sites in the reaction rate. In this chapter, our main approach is simulating such a system in order to study the reactivities of different patterns on the surface. We want to consider some patterns and compare the performance in the same thermodynamic condition using Kinetic Monte Carlo Simulations. In this chapter we will test the predictions of continuum approach and observe the breakdown and limits of the continuum approximation as the size of active sites approaches the mean free path of the gas: $L \rightarrow \lambda[86]$. We also investigate the effect of patterning of active sites on the catalyst surface (e.g. checkerboard and stripes). The continuum theory predicts that for a given density of active sites, the performance of the catalyst depends only on the period of the pattern $L$ and not the particular pattern. The kinetic Monte Carlo methods allow us to test this prediction in the limit where $L \sim \lambda$. 
Before we start looking at the simulation of the chemical processes of heterogeneous catalytic surface, we explain briefly about the tools and concepts that we need for our simulation in this chapter.

\subsection{Numerical Simulation of Diffusion Limited Catalysis}

In this chapter, we applied the Monte Carlo simulation method. The Monte Carlo method is a good technique for calculating averages in a multi-particle system.

In this section, we model the diffusion of the reactant as a random walk. For a system comprises an ideal gas in a non-equilibrium state this can be simulated by the Kinetic Monte Carlo method. Since the diffusion of the reactant near the catalytic surface is not uniform, the Kinetic Monte Carlo method will use different rates for different processes. We will explain this method in the following section.

\subsubsection{Random walk model}

A drunken person in a city is a common example of the random walk model. A drunk person in a city has no idea where he is coming from or is going to, therefore, his chosen direction is random. Each step is considered as an event in Monte Carlo simulation. If we consider each drunk person as a particle, we have so many of these drunk people moving around randomly in a city. We assume at each time, only one of these random walkers changes its place based on the random decision. In the long time limit, their average position will tend to be zero and their average displacement will be close to the origin.

The simplest possible case is a one-dimensional random-walker with steps of equal lengths of lattice space and time intervals. At each step, which is equal to the time interval of event $\left(t_{i}\right)$, the random walker will either move right or left with equal probability.

$$
\left\{\begin{array}{llll}
+l & \text { with } & 50 \% & \text { Probability } \\
-l & \text { with } & 50 \% & \text { Probability. }
\end{array}\right.
$$




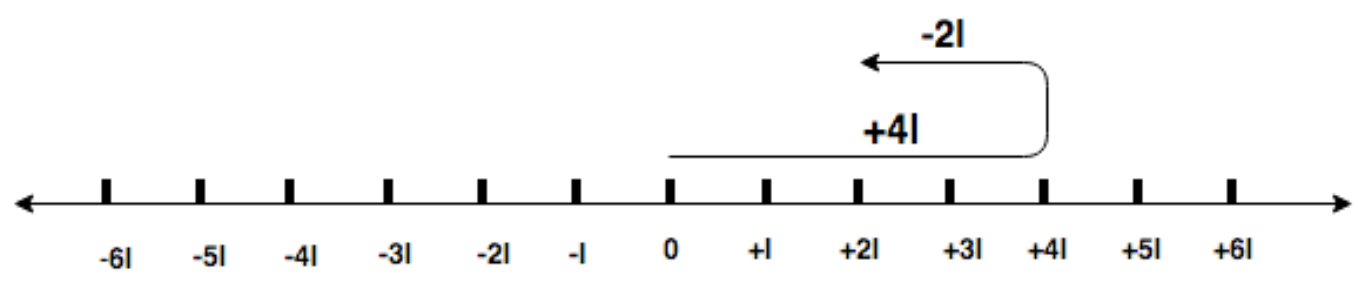

FiguRE 3.1: The random walker pathway in one-dimensional space. This figure shows the walker first started from the origin. His first four steps randomly were in the right direction and his next two steps were in the left direction. The right direction is assigned as the positive direction and the left direction is assigned as the negative direction.

By counting the total number of steps with their direction we can obtain the ultimate point of the particle, $r(N)$ :

$$
r(N)=\sum_{i=1}^{N} l_{i}
$$

The average of this point in a long time limit is zero.

$$
\langle r(N)\rangle=0
$$

In many Monte Carlo simulations, the time between two steps depends on the time that is taken for a process to be finished. Therefore, for systems that have the limitation of space or particles, as the system goes toward the saturation or lack of one element, the interval time between two events or process takes longer. In our system time is varied from nanosecond to a microsecond.

\subsubsection{Continuum limit}

In the limit of vanishing lattice spacing $l$ and time interval, the random walk model approaches a continuum diffusion model. The dynamics of random-walker can be explained by the master equation of motion. In one dimension, the probability of a particle being at a certain point depends on its probability of being in the nearest neighbors of this point at last time step: 


$$
P(i, N)=\frac{1}{2} P(i+1, N-1)+\frac{1}{2} P(i-1, N-1)
$$

where $i$ refers to the site number and $N$ shows the number of steps in the MC simulation. The master equation for two dimensions will have 4 terms $i, j$ unit vectors, and in three dimensions 6 terms with $i, j, k$ elements.

The ultimate point will be:

$$
\left\{\begin{array}{l}
t=N \tau \\
r=i l
\end{array}\right.
$$

where $\tau$ is the time step and $\mathrm{t}$ is the total simulation time. We normalize our equation so the probability integrated to one:

$$
P(r / l, t / \tau)=\frac{1}{2} P(r / l-1, t / \tau-1)+\frac{1}{2} P(r / l+1, t / \tau-1)
$$

By multiplying $l$ to both sides and factoring $\tau$ and substracting $P(r, t-\tau)$ :

$$
\frac{P(r, t)-P(r, t-\tau)}{\tau}=\frac{P(r-l, t-\tau)+P(r+l, t-\tau)-2 P(r, t-\tau)}{2 \tau}
$$

which leads to:

$$
\left\{\begin{array}{l}
P(r, t-\tau) \approx P(r, t)-\tau \frac{\partial P(r, t)}{\partial t} \\
P(r \pm l, t-\tau) \approx P(r, t) \pm l \frac{\partial P(r, t)}{\partial t}+\frac{1}{2} \frac{\partial^{2} P(r, t)}{\partial r^{2}}-\tau \frac{\partial P(r, t)}{\partial t}
\end{array}\right.
$$

by substituting Eq. 3.8 into Eq. 3.7, we obtain the equation of motion of a particle:

$$
\frac{\partial P(r, t)}{\partial t} \approx \frac{l^{2}}{2 \tau} \frac{\partial^{2} P(r, t)}{\partial r^{2}}
$$

where $\frac{l^{2}}{2 \tau}=D$. From Eq 2.24 the probability distribution of a particle at $(\mathrm{r}, \mathrm{t})$ becomes:

$$
P(r, t)=\frac{N}{\sqrt{4 \pi D t}} e^{\frac{-r^{2}}{4 D t}}
$$


Where $2 D t=\left\langle r^{2}(t)\right\rangle$ from Einstein relation. This is the solution of (Eq. 3.9)and is similar to Gaussian form of distribution. There are different types of modified randomwalk based on physical conditions of systems. Self-avoiding random walk, persistent random walk and restricted random walk are examples of this modified model [87].

\subsubsection{Kinetic Monte Carlo simulation method}

In the MC simulation we try to follow the dynamics of a system where events such as growth and diffusion are stochastic and depend on various numbers of random factors that are modeled in a system. Different physical phenomena can be modeled with the MC simulation from random hopping of atoms to the growth of colloidal particles. Most statistical mechanics problems that involve sampling of the range of phase space for exploring certain properties can be modeled with the MC simulation. Sometimes beside $\mathrm{MC}$, we add up further modifications in order to reach the equilibrium state faster such as the coarse-graining model in which we neglect those interactions that are not affecting statistical properties of the system and we rescale all the system's interaction scales to the minimum scale that needs to be considered in the system. In this thesis, we shall focus on statistical and kinetical properties of the system, therefore, applying Kinetic Monte Carlo (KMC) would be the best solution. Although there are different kinds of simulation methods for simulating atomic scale systems, they are not always effective[88]. This diffusion can be explained as a move from the old state to the new state based on conditional probability density. This probability density will depend on the energy difference between the old state and the new state. The energy difference value will be compared with a random number generated in each MC step that can lead to two approaches: sampling with rejection or rejection free sampling. If the random number is greater than the potential energy function, the system cannot move and needs to find another path for its transition, whereas in rejection sampling the transition will be rejected and no move will occur at that MC step. Applying either approach depends on the time evolution of the system. In this thesis, we applied the KMC method with rejection sampling. The KMC method is essentially the same as the dynamic Monte Carlo method and the Gillespie algorithm[89]; the main difference is in terminology and usage areas. 


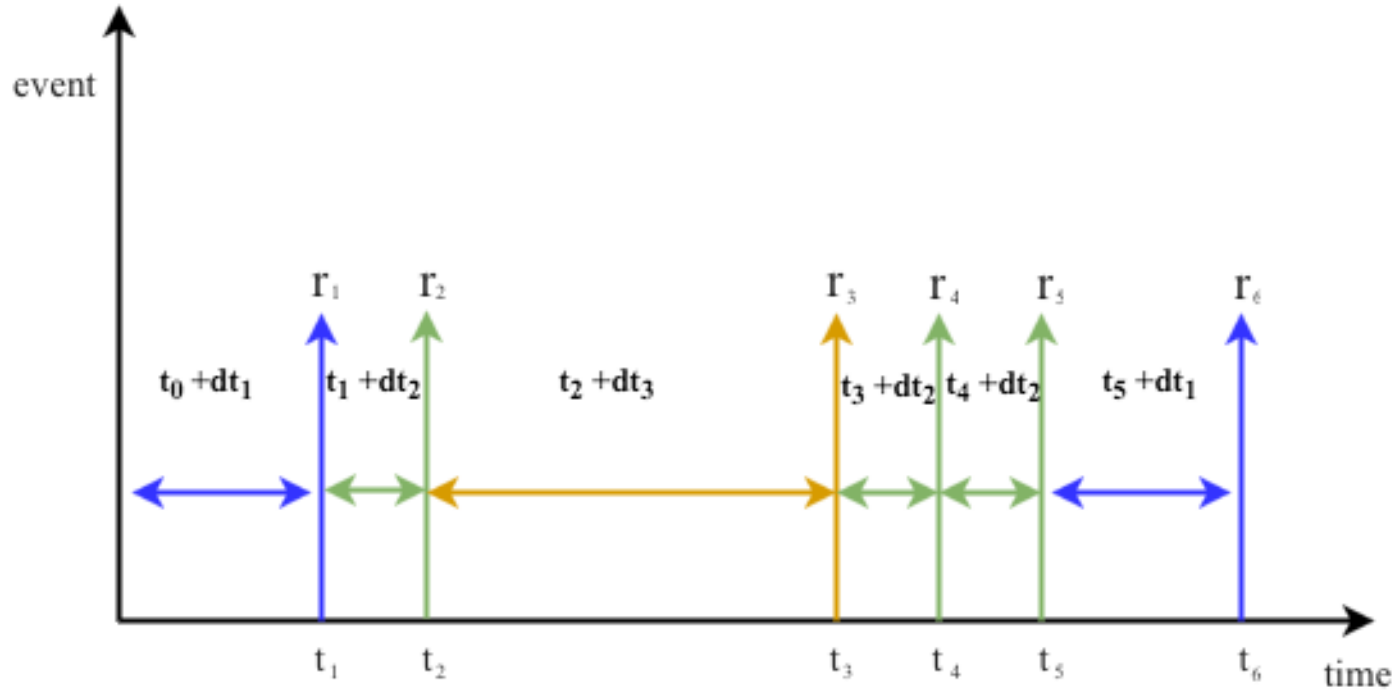

Figure 3.2: Time of occurrence of each event based on time the interval. Three different types of process $\left(r_{1}, r_{2}, r_{3}\right)$ happen at different times. Some event e.g $r_{3}$ takes longer than $r_{2}$ to be completed as an event.

One of the advantages of using the KMC method is its ability to simulate on large timescales. Whereas in most of the simulation methods, for example, Molecular Dynamics (MD) simulation, accurate dynamical integration requires short time steps to cover all the particular events, while we would like them to happen on a larger timescale. This leads to timescale problem. The goal of this section is to become familiar with the concepts that underlie the Kinetic Monte Carlo method. The algorithm that we present in this thesis has been used for crystal growth and is applicable to a broad range of other systems that are modeled as heterogeneous random walks. Usually, when the diffusion process occurs in discrete phase space and continuous-time Markov process, KMC simulation offers distinct stochastic events during the simulation, which underlies on the spatially heterogeneous surface.

Generally, we have a sequence of states $\left\{x_{i} \in X\right\}$ based on the total state space $\{X\}$. At each time a transition through one state can occur[90]. As a result, the dynamics of the system can be categorized into a set of transition from state $x_{i}$ into another possible state $x_{j}$. Usually, the biased probability is such that system tends to transit to the lower energy of states.

$$
r_{i j}\left(x_{i} \rightarrow x_{j}\right)
$$

which $r_{i j}$ is a rate of one single process that is from site $i$ to another site $j(i \rightarrow j)$ so the total rate is given by: 


$$
R_{i}=\sum_{j=1}^{i} r_{j}
$$

for $i=1, \ldots N$ where $N$ is the total number of transitions. Denote $R=R_{N}$ Therefore, the transition probability is given by:

$$
p_{i j}=\frac{r_{i j}}{R}
$$

and total probability is given by:

$$
P=\sum_{i, j} p_{i j}
$$

Each sequence of transition happens in an inhomogeneous Poisson process. Some of the states at some points are not accessible, and therefore, system has to restrict its option to those rates that are accessible for that certain energy and time interval.

$$
f(t)=r e^{-r t}
$$

where $r$ is the transition rate of the system at its current place. $t$ is the life time event. The equation gives the probability of transition that occurs at each waiting time distribution. The key point of all is that there are independent and there is no correlation between them. At each time step we find an event to carry out by finding the $i$ for which:

$$
r_{i-1}<u R<r_{i}
$$

where $\mathrm{u}$ is a random number between 0 and 1 For the next step we update the time by $t+\tau$. The occurrence of an event may affect the future rate of events since the total number of available states and particles will be changed after that step. Therefore, we need to recalculate each $r_{i j}$ and time by:

$$
\tau=\frac{-\log u}{R}
$$




\subsection{Simulation of heterogeneous system}

To test the theory developed in the previous chapter in section (2.4), we have implemented the Langmuir adsorption model (processes (1-4)) together with the lattice diffusion of the gas A near the surface using the kinetic Monte Carlo simulation method.We consider a cubic $40 \times 40 \times 40$ lattice within a box with periodic boundary conditions in the $x$ and $y$ directions, and a catalytically active surface at the boundary $z=0$. The particles diffuse on the lattice, where the lattice constant is chosen to be equal to the mean free path $\lambda$ of the gas A. At the top of the box, we impose a reflecting boundary condition. The total number of particles in the simulation box is kept constant at 1600 by replacing particles that are converted with new particles that potentially can adsorb on the surface, from the top of the simulation cell. In the simulations presented here, we have neglected process (2) (i.e. we have set $k_{2}=0$ ) and consider that the process (4) is so rapid that it happens instantaneously (i.e. once conversion occurs we simply delete the particle from the catalyst surface). There is no interaction between the particles, except that only one active site on the surface can be occupied at any given time. This leaves three rates to consider in the simulations: $\tau_{D}^{-1}=D / \lambda^{2}$, the rate of diffusion in the gas; $k_{1} Q$, the rate of adsorption; and $k_{3}=\tau_{c}^{-1}$, the rate of conversion of adsorbed particles. The time constant $\left(\tau_{D}\right)$ is the mean free time of the diffusing gas particles, which we chose as our unit of time. The time constant $\left(\tau_{c}\right)$ is the relaxation time of the catalytic conversion process, which occurs only at occupied actives sites. These active sites on the catalyst surface $(z=0)$ are arranged in a periodic pattern or randomly distributed to achieve a specified surface coverage. We use a rejection-free kinetic Monte Carlo method to evolve the system to a steady-state, which typically requires $10^{6}$ Monte Carlo steps. This was checked to ensure that the particle flux at the catalyst surface had settled to a steady value and that the density gradient of the particles was approximately constant in the $z$-direction. We have considered spatial variations in $k_{1}(x, y)$ over the surface while considering $\tau_{c}$ to be constant everywhere else. We have tested patterns of different motif and coverage, together with variations in the period $L$. When $\tau_{c} \ll \tau_{D}$ the simulations can be used to test the expressions for the effective activity under diffusion-limited conditions given in the previous section (equations (2.39) and $(2.42))$. 


\subsubsection{Results}

We first consider the continuum limit, $L \gg \lambda$, with $\tau_{c} \ll \tau_{D}$, where we would expect (2.39) to hold for finite values of $k_{1}$. In Figure 3.3 and 3.4 we consider the effective activity of a series of checker board patterns (such as those in the schematic in Fig. 2.7), where the period $L$ is scaled from 2 to $20 \lambda$ and the surface coverage is varied from $\phi=0$ to 1 . All sites on the surface are active, but some have absorption probability $k_{1}=1.0$ (with surface coverage $\phi$ ) while others have $k_{1}=0.5$ (with surface coverage $1-\phi$ ). We chose $\tau_{c}=0.1 \tau_{D}$ to be constant over the entire surface. When $l \sim \lambda / k_{1} \ll L$ we would expect equation (2.39) to hold. As shown in the figure, as $L$ gets larger, the effective activity, and its dependence on the surface coverage, indeed approaches that predicted by equation (2.39). This illustrates the effectiveness of the homogenization approach in the continuum limit. Away from the continuum limit, when $L \sim l$ the theoretical expression underpredicts the effective reactivity. In fact, as $L \rightarrow \lambda, k_{e f f}$ is found to increase. In other words, for a fixed average coverage of active sites, surfaces with sites dispersed on scales comparable to $\lambda$ are found to be more effective at capturing reactant than surfaces where sites are more concentrated.

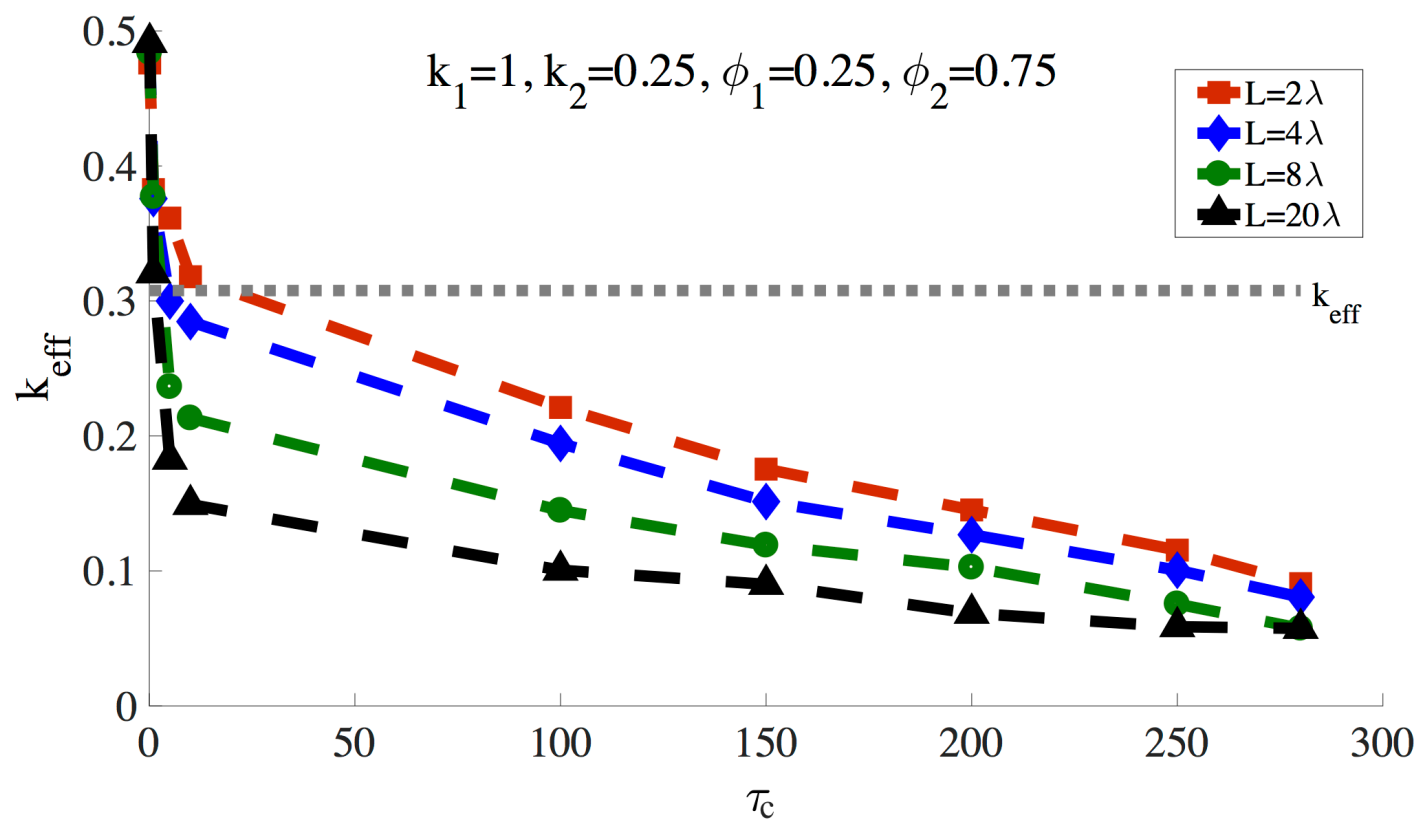

FIGURE 3.3: The figure compares the effective activities for a range of different domain sizes as a function of the surface coverage with effective reactivity predicted by equation (2.39)and surface coverage of active sites with $k_{1}=1.0: 0$ of $\phi=25 \%$ and $k_{2}=0.25$ elsewhere as $\tau_{c}$ is varied. For length scales $L \sim \lambda$, the effective activity exceeds that predicted by (2.39) but as $L$ gets larger, the effective activity is well described by equation (2.39). 


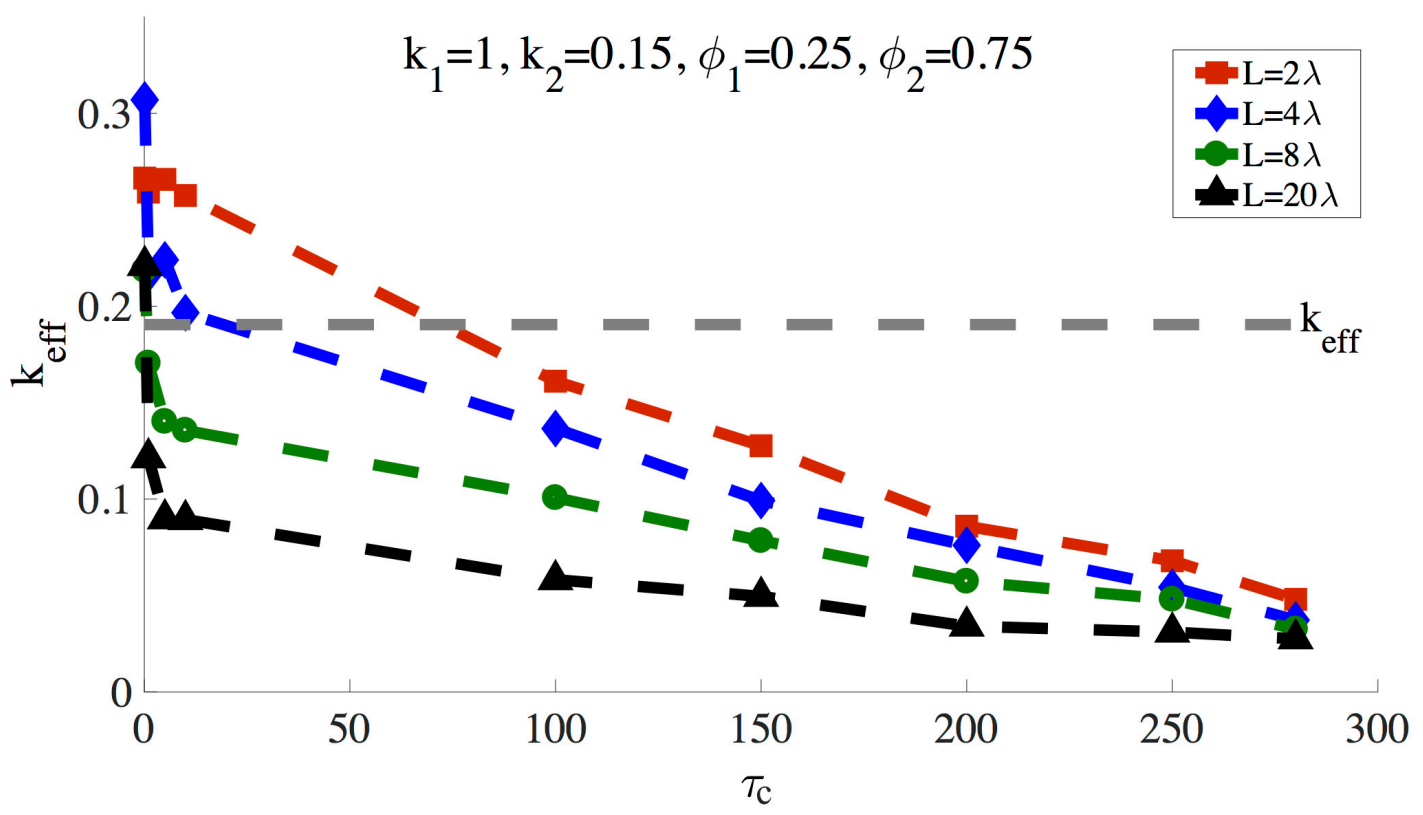

Figure 3.4: The graph compares the effective activities for a range of different domain sizes as a function of the surface coverage with effective reactivity predicted by equation (2.42) and surface coverage of active sites with $k_{1}=1.0: 0$ of $\phi=25 \%$ and $k_{2}=0.15$ elsewhere as $\tau_{c}$ is varied. For length scales $L \sim \lambda$, the effective activity exceeds that predicted by (2.42) but as $L$ gets larger, the effective activity is well described by equation (2.42).

It is also interesting to consider cases where $k_{1}=0$ on some parts of the surface, while $k_{1}$ is finite elsewhere. In Figure 3.4 we again consider the effective activity for a series of checker board patterns as the period $L$ increases from $\lambda$. Two curves are shown in the figure: in the first, $k_{1}=1.0$ on active sites, which have a coverage $\phi=0.25$, while in the second, $k_{1}=0.8$ on active sites, which have a coverage of $\phi=0.5$. On all other sites $k_{1}=0$, so adsorption is not possible. Again $\tau_{c}=0.1 \tau_{D}$ over the entire surface, although now process (3) only occurs on active sites. In this case, we see that as $\lambda / L \rightarrow 0$ the effective activity $k_{\text {eff }}$ approaches the value given by equation (2.42). Although $L \gg \lambda$ in this limit, over much of the surface $L \ll l \sim \lambda / k_{1}$ as $k_{1}=0$ and equation (2.42) seems to provide a good description of the effective activity. Away from the continuum limit, equation (2.42) again underestimates the effective activity of the surface. 


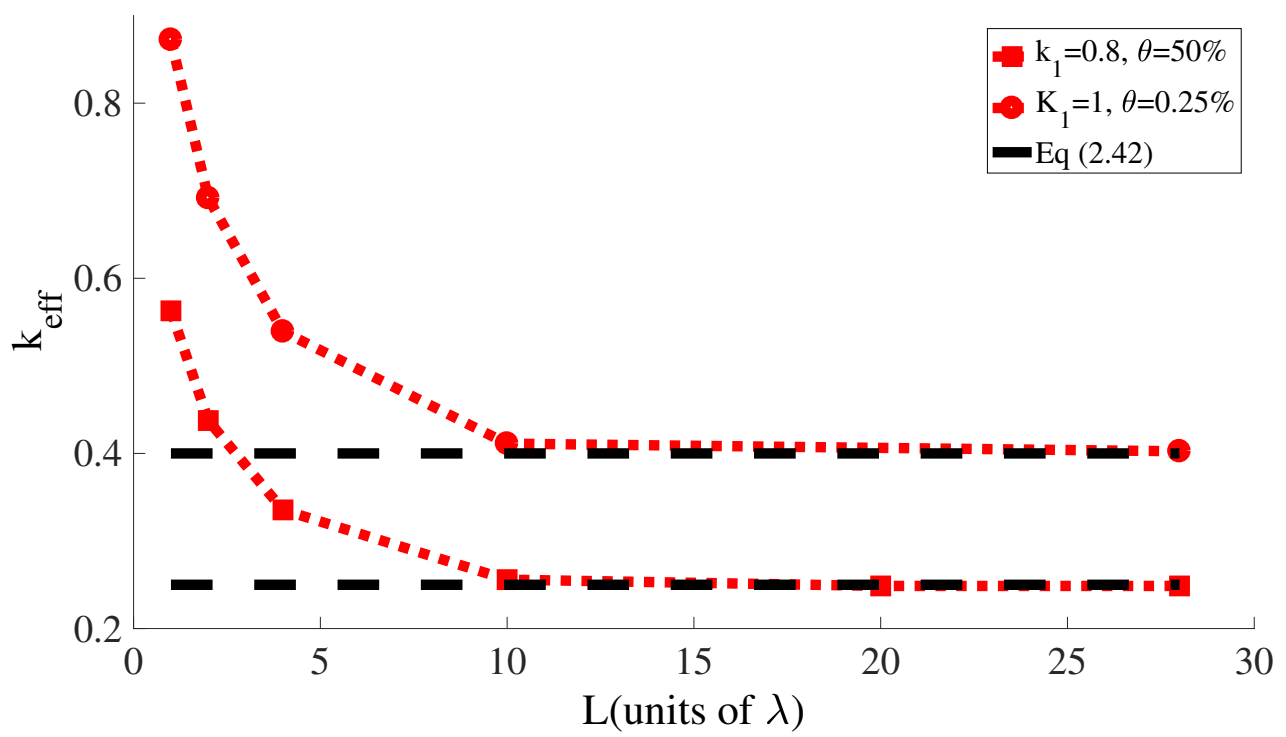

Figure 3.5: The relationship is shown between the effective activity $k_{e f f}$ and the domain size $L$ is shown for the case where $k_{1}=0.8$ at $50 \%$ coverage (and $k_{1}=0$ elsewhere) and the case where $k_{1}=1.0$ at $25 \%$ coverage. The patterns here have a checkerboard character. The effective activity in the simulations approaches that predicted by equation (2.42) as $\lambda / L \rightarrow 0$ but exceeds this as $L \rightarrow \lambda$.

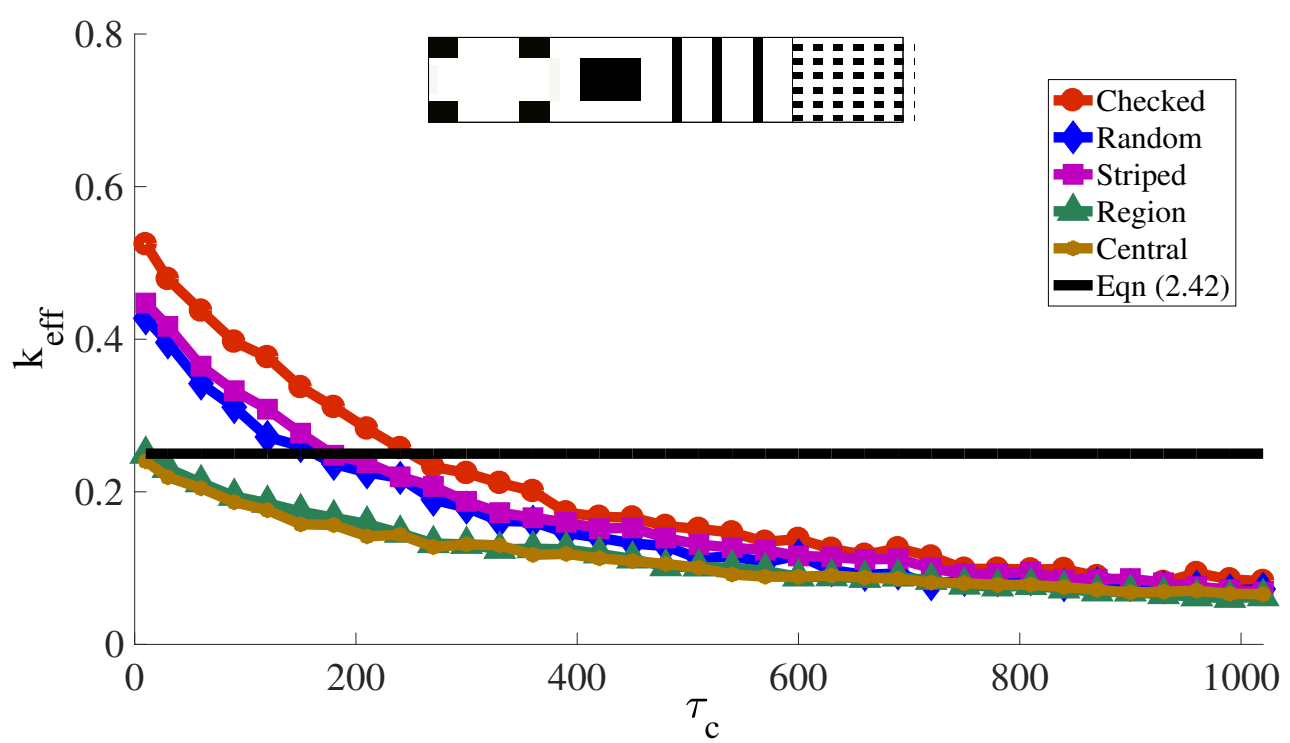

FiguRE 3.6: The figure shows the effective activity of different patterns with a surface coverage of active sites with $k_{1}=1.0$ of $\phi=25 \%$ and $k_{1}=0.0$ elsewhere as $\tau_{C}$ is varied. In the range of $0<\tau_{C} \ll \tau_{D}$, the larger length length scale regions approach agreement with equation (2.42). For the patterns with periods comparable to the mean free path $(\lambda)$, the effective activity exceeds the limit predicted by equation $(2.42)$ as $\tau_{C} \rightarrow \tau_{D}$. 


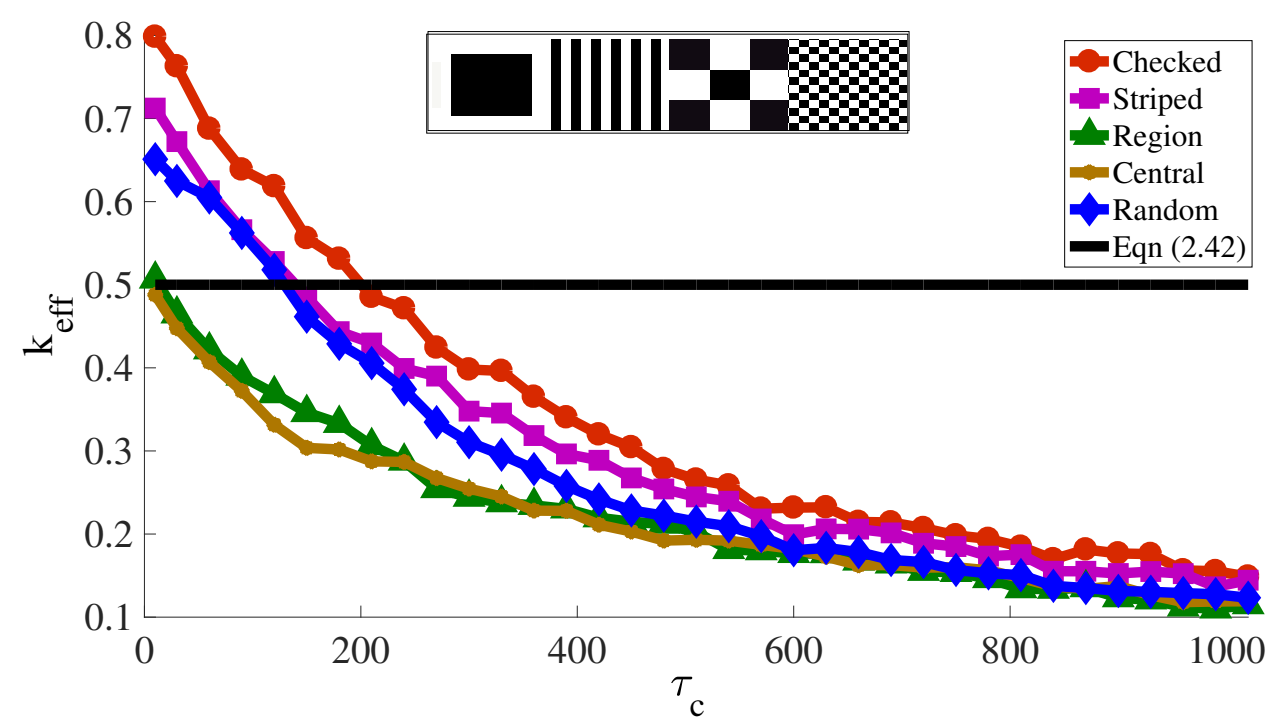

Figure 3.7: The figure shows the effective activity of different patterns with a surface coverage of active sites with $k_{1}=0.8$ of $\phi=50 \%$ and $k_{1}=0.0$ elsewhere as $\tau_{C}$ is varied. In the range of $0<\tau_{C} \ll \tau_{D}$, the larger length length scale regions approach agreement with equation (2.42). For the patterns with periods comparable to the mean free path $(\lambda)$, the effective activity exceeds the limit predicted by equation $(2.42)$ as $\tau_{C} \rightarrow \tau_{D}$.

Finally, we have considered the effect of different patterns, as well as the case where $\tau_{c}>\tau_{D}$, which is not covered by the theory developed in the previous chapter. We have considered a variety of patterns, as shown in the inset of figures 3.6, 3.7, including a selection of checkerboard-type patterns, a pattern where active sites are randomly distributed over the surface, and stripes. As in the previous case, we find that for the larger domain sizes the effective activity is given by equation (2.42) for small $\tau_{c}$, and that the effective activity of the patterns with smaller length scales exceed those predicted by the theory. As $\tau_{C}$ increases the effective activity decreases, as one might expect as the activity becomes reaction-limited rather than adsorption or diffusion-limited. Also note that the effective activity is pattern dependent, with the checked pattern with the period $L=2 \lambda$ outperforming the stripes and the random pattern, which in turn outperform the patterns on larger length scales.

\subsection{Discussion and conclusions}

We have found that the two approximate expressions (2.39) and (2.42) for the effective catalytic activity of a surface with a heterogeneous distribution of catalytically 
active sites match the simulations well in the limits where they are expected to be valid. For distributions of active sites with length scales below the continuum limit we find that these expressions underestimate the effective activity. In air, under standard conditions, the mean free path of a gas molecule is of the order of $100 \mathrm{~nm}$. Under these conditions, we would expect that the continuum diffusion equation (2.30) provides a good description of variations in the density of the gas at the catalyst surface on scales greater than $100 \mathrm{~nm}$. Variations in the distribution of active sites below this scale would produce variations in the density of the reactant gas that would not be captured well by the continuum diffusion equations, as demonstrated by the KMC simulations presented here. Nonetheless, the effects of variations in the distribution of active sites above this scale should be able to be captured by the continuum approach so it is interesting to consider the implications of the continuum theory. Consider a distribution of small but high activity particles (metal nanoparticles of diameter $10 \mathrm{~nm}$, say, with $k_{1}=k_{a}$ ) dispersed on larger, less active oxide particles (e.g $1 \mu \mathrm{m} \mathrm{TiO} 2$ particles, with $k_{1}=k_{d} \ll k_{a}$ ) for instance[91]. Here we might find a variation of activity on length scales, $L$, of greater than $100 \mathrm{~nm}$, so the continuum theory would be expected to be valid and equation (2.39) should hold. In this case, small particles play the role of active sites and their fractional enhancement to the effective activity $\left(k_{e f f} / k_{d}\right)$ is proportional to $1 /(1-\phi)$. The small particles essentially consume all the reactant they encounter, so it is their coverage rather than their activity that determines the enhancement to the effective activity of the larger particles.

In summary, we have applied a mathematical homogenization approach (Chapter2) together with KMC simulations (This chapter) to investigate the effect of scale and patterning on the effective activity of catalytic sites on a heterogeneous catalyst operating under diffusion-limited conditions. The KMC simulations show that in the continuum limit the kinetic theory of gas works well where there is a high number of particles diffusing over the active sites. In particular, in the limit where the mean free path is much less than the scale of patterning of catalytically active sites, the effective rate constant is found to be equal to the harmonic mean of the rate constant over the surface. However, as the length scale of the patterns becomes comparable to the mean free path length, the effective activity of the system can exceed the theoretical limit suggested by the continuum theory. We expect that this work will have implications for the design and use of nanostructured catalysts that need to operate under diffusion-limited conditions. There 
are so many systems that they perform chemical process under high temperatures, for such a system the mean free path of the particles are larger than the ideal gas, therefore, the system is in diffusion-limited condition. 


\section{Chapter 4}

\section{Introduction to Polymer Physics}

From a physical point of view, a polymer chain consists of a set of units called monomers, which are chemically bonded with hydrogen or covalent forces. Real examples of polymer chains include DNA molecules or proteins[92]. DNA molecules are very important for life, their primary structures are linear (multiple chemical units) composed of monomers (single chemical units), called nucleotides, as are proteins which consist of amino acids (monomers). The basic properties of a polymer are a consequence of the size of the polymer chain and the size of monomers (which correspond to the volume of space excluded by the monomers). This controls other characteristics of the polymer chain such as extensivity, flexibility, and conformation of the polymer[93]. Generally, the monomer structure and the way that they are assembled determine the physical behavior of the polymer (Figure. 4.1). The unit of repetition for some polymer molecules can consist of more than one type of monomer. When there are two types of monomer in one polymer chain, it is called a diblock copolymer $(A-B)$. When the arrangement of monomer $A$ and monomer $B$ is random, the chain is called a random copolymer. A polymer with three types of monomer is called a triblock copolymer. In contrast a polymer with one type of monomer is called homopolymer chain (Figure. 4.2)[94].

From a structural point of view, we can consider three types of polymer chain: linear, branched and cross-linked. Linear polymer chains consist of one single chain with repeated monomers, whereas a branched polymer has one backbone and several branches. The combination of single chain makes cross-link polymer with several crossed joints. There are other ways to classify polymers, e.g. whether they are organic or 


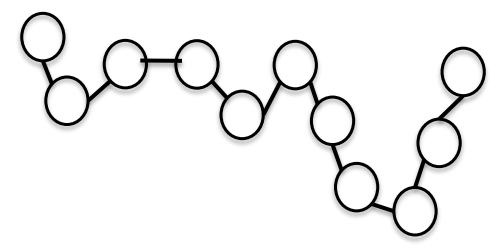

(a)

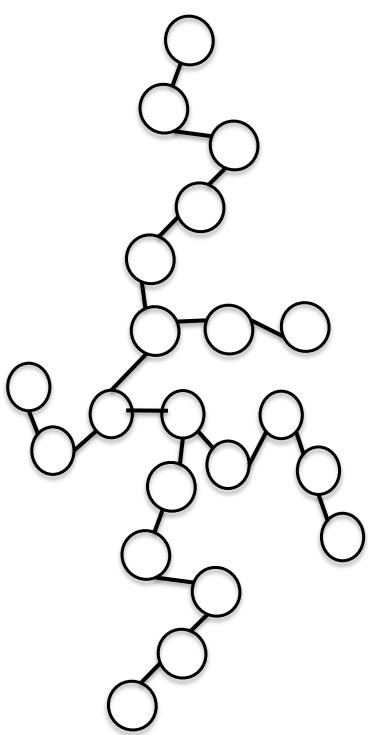

(b)

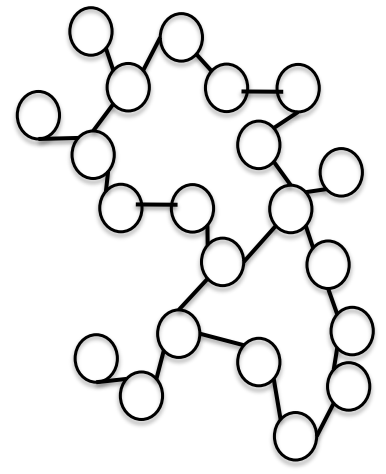

(c)

Figure 4.1: A general structure of single (a) , branched (b) and cross-linked poly$\operatorname{mer}(\mathrm{c})$.

inorganic, or anionic or cationic. However, the most useful way for our purposes is based on their mechanical and thermodynamic behavior in solution. Since polymers are quite sensitive to physical and thermodynamic conditions, they behave differently under different physical conditions[95].

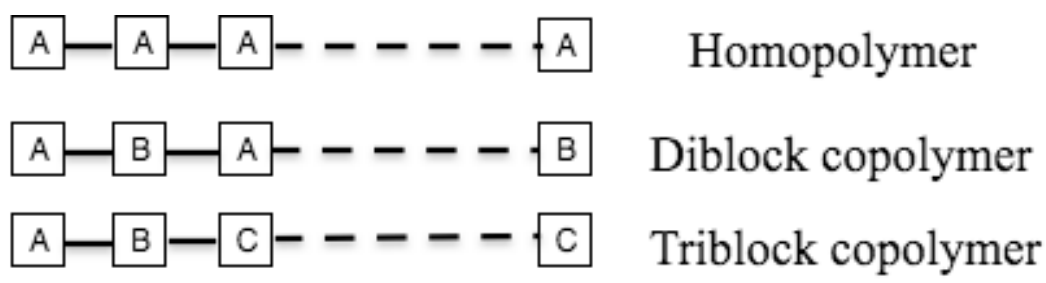

FIgURE 4.2: This figure shows the periodic repetition of the single polymer chain that can be homopolymer (only one type of monomer), diblock copolymer (two types of monomer) or (triblock polymer).

A polymer chain in solution can change its shape dynamically and its instantaneous shape is called a conformation[96]. If we fully stretch a polymer chain, the distance between two ends is called the contour length. However it is quite hard to find a polymer 
chain fully stretched in a solution since it is highly fluctuating. Instead, its configuration is usually crumpled and has a random coil conformation[97].

In this chapter, we will study how physical conditions such as temperature of the system can lead to applying a different numerical models. Moreover, how to transit from one model to another when the physical condition of the medium changes, will be discussed later.

\subsubsection{Overview of Polymer models}

In order to study the rheology of polymers, their physical structures are more important than their chemical components, since the deformation and flow in the solution can be changed under different thermodynamic conditions. However, in this thesis, we are interested in adsorption of the polymer chain on a surface. Therefore, we need to study the interaction of polymers and the surface. In order to study the microscopic behavior including their governing potentials near the surface we apply a coarse-grain model. The coarse-grain model helps us to limit the number of degrees of freedom and the motions. The thing that is important in coarse grain model is the size correspondence features. Scaling plays a key role in simulating a polymer by this model. In 1946 Kremer defined a model called the Free Joined bead-rod chain in which he introduced the monomers of the polymer as a bead and their connections are called rods. The size of the rod corresponding to one step is called a Kuhn length, which is the smallest connection length between two monomers[98]. Several coarse-grained models have been applied to predict the physical behaviors of polymers based on physical characteristics such as length, volume, and size of the chain[99]. The bead-spring model, bead-stick model, and pearl necklace model are illustrated in Figure. 4.3.

In the bead-spring model, the beads are monomers that are connected by springs. Connection by a spring allows the bond between monomers to fluctuate over a certain range of lengths. In a bead-stick model, monomers are connected through a single rod, in either freely jointed-chain or a freely rotating chain. In the freely jointed chain the direction of bond angles are totally unrelated and random, whereas in the freely rotating chain the correlation between the bonds can be defined as[100]:

$$
\left\langle\overrightarrow{r_{i}} \cdot \overrightarrow{r_{j}}\right\rangle=b^{2} \cos \theta^{|i-j|}
$$




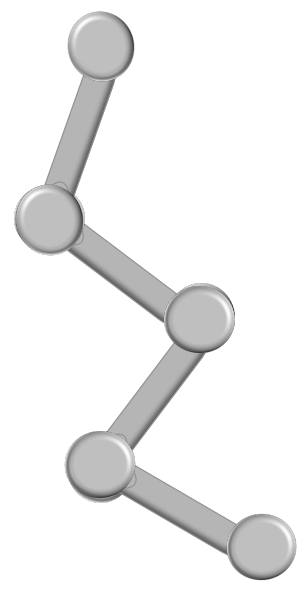

(a)

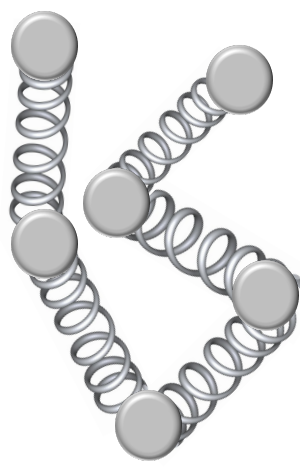

(b)

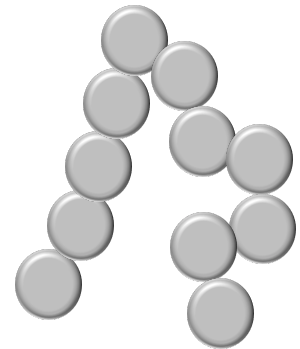

(c)

FiguRE 4.3: Based on the bond length between monomers and their flexibilities, we can construct each polymer chain with these three types of models Figure (a) is a bead-stick model which models a rigid polymer chain Figure (b) is a bead-spring model which is a model for a semiflexible polymer chain. Figure (c) is pearl-necklace model and is suitable for a flexible polymer chain.

$r_{i}$ and $r_{j}$ are the bond vectors of $\mathrm{i}$-th monomer and $\mathrm{j}$-th monomer. $\theta$ is the angle between the two adjacent bond vectors. $b$ is the Kuhn length between the two monomers. In very high temperatures we can apply the pearl-necklace model. In the pearl-necklace model, monomers are always in contact with their two nearest neighbors. In very low temperatures we apply rod model when monomers have a maximum distance from each other. Pearl-necklace model is similar to rod model with rod length equal to the diameter of monomers or beads[101].

\subsubsection{Scaling of Polymer Chain}

\section{End-to-end distance and Centre of mass}

In previous sections, we modeled a polymer as $\mathrm{N}+1$ units with $N$ joints where the position of these joints is denoted by $\vec{r}_{i}(i=0,1, \ldots N)$, and therefore, the end-to-end vector is then given by $R[102]$ :

$$
R \equiv \vec{r}_{N}-\vec{r}_{0}
$$


and consequently the mean squared of the end-to-end vector is:

$$
R^{2} \equiv\left\langle\left(r_{N}-r_{0}\right)^{2}\right\rangle
$$

It is quite common to use the center of mass of the polymer chain in order to find some information about the elasticity and the flexibility of the chain. If we neglect the mass of monomers, the center-of-mass is given by:

$$
r_{G}=\frac{1}{N+1} \sum_{i=0}^{N} r_{i}
$$

With this result we can find the radius of gyration:

$$
R_{g}^{2}=\left\langle\frac{1}{N+1} \sum_{i=0}^{N}\left(r_{i}-r_{G}\right)^{2}\right\rangle=\frac{1}{N+1} \sum_{i=0}^{N}\left\langle\left(r_{i}-r_{j}\right)^{2}\right\rangle
$$

which we will use this result in chapter 5 and we measure the centre of mass of the polymer chain in adsorbed and desorbed states.

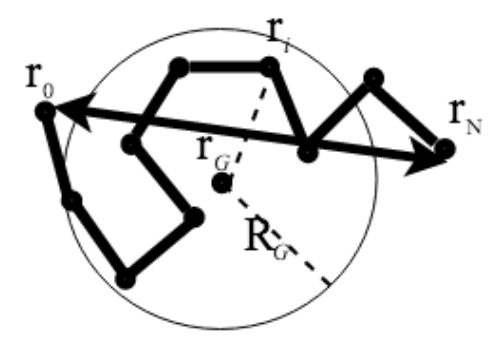

Figure 4.4: This Figure shows end-to-end vector, the centre of mass and the radius of gyration in a single polymer chain.

\subsubsection{Flory Exponent}

When the length of the polymer chain increases, the swelling of the chain becomes important. In order to obtain a precise value for the radius of gyration of the ideal chain, we need to consider the exponent of increasing the dimension of the monomers that can change the strength of swelling of the chain. The exact value of the radius gyration and its exponent is given by renormalization group theory. However, this approach is more complicated than the Flory approach. Flory could estimate this exponent in a simple 
way. Therefore this approach is called the Flory exponent:

$$
R_{g} \cong b N^{\nu}
$$

where $\nu$ is the Flory exponent, $\mathrm{N}$ is the total number of monomers and $\mathrm{b}$ is the Kuhn length. The value of the Flory exponent, and consequently the radius of the gyration, is related to the physical condition of the polymer in the system. For example, depending on the temperature of the system, the polymer can be in different phases. These phases can be divided into three main categories: Collapse, Ideal and Extended. In the collapse phase, the attraction between monomers is such that monomers tend to stay as close as possible, whereas in extended phase, the repulsive interaction between monomers does not let them get close together. In each phase, the Flory exponent has different values. In the collapsed phase the Flory exponent is proportional to $\frac{1}{d}$, where $\mathrm{d}$ is the dimension of the system and in extended phase is $3 / d+2$ for $d \leq 4$ and $1 / 2$ for $d>4$. The phase between collapsed and extended is called the ideal phase, and the Flory exponent is equal to $1 / 2$. In this thesis we assume our system is in the ideal state; however, there are some possibilities of transit to other states. In this research, we also consider hightemperature phases where the polymer is in the collapse phase and as the temperature goes down the polymer will become more extended. Therefore in a system with a fixed number of monomers, the size of the radius of gyration will change over the range of temperatures.

\subsection{The Linear Polymer Chain Model}

\subsubsection{Continuous Space Model}

The general concept behind the continuum models for polymers is that the probability distribution of the long polymer chain should be universal, no matter what type of monomers are in the structure [103]. In 1965-66 S.F. Edward considered a unique simplified model for polymers and this was the first model that introduced the idea of universal properties [102]. In this model, we consider the polymer chain to be a string described by features like the length scale of bonding between monomers $b$ and the Flory exponent $\nu$ as a viscosity indicator[104]. If we consider cross-sectional path length $(\Delta l)$, we can show the total path length will become $\mathrm{R}=\mathrm{N} \Delta l$. One should also take the limit 
that the number of monomers $\mathrm{N} \rightarrow \infty$. The advantage of applying continuum model for a long polymer chain is that by having the information of a small part of distribution we can integrate the probability distribution of the whole chain from point 0 to $\mathrm{L}$, which $\mathrm{L}$ is the total length of the polymer.[105],[106]. Comparing the distribution of continuous with the discrete system, we first start with the potential energy of the bead-spring model in the discrete system. If we assume the beads are in the position of $r_{0}, r_{1} \ldots r_{N}$ the potential energy of the chain is given by potential energy of the spring constant force $k_{s}$ with:

$$
U\left(r_{0}, r_{1, . .}, r_{N}\right)=\frac{1}{2} k_{s} \sum_{n=1}^{N}\left(r_{n}-r_{n-1}\right)^{2}
$$

For certain arrangments the Boltzmann distribution function of the chain is given by:

$$
\exp \left[U\left(r_{0}, r_{1, . .}, r_{N}\right) / k_{B} T\right]=\prod_{n=1}^{N} \exp \left[\frac{k_{s}}{2 k_{B} T} \sum_{n=1}^{N}\left(r_{n}-r_{n-1}\right)^{2}\right]
$$

The distance between two monomers are defined as $\Delta l=b$ and $b^{2}=\frac{3 k_{B} T}{k_{s}}$. Thus:

$$
\exp \left[U\left(r_{0}, r_{1, . .}, r_{N}\right) / k_{B} T\right]=\exp \left[\frac{3}{2 b \Delta l} \sum_{n=1}^{N}\left(r_{n}-r_{n-1}\right)^{2}\right]
$$

by rewriting $r_{n}-r_{n-1}=\partial l$ and considering $n$ to be continuous.

$$
\lim _{N \rightarrow \infty} \exp \left(-\frac{3}{2 b} \sum_{n=1}^{N}\left(\frac{\partial r}{\partial n}\right)^{2} \Delta n\right)=\exp \left(-\frac{3}{2 b} \int_{0}^{\mathrm{L}}\left(\frac{\partial r}{\partial l}\right)^{2} d l\right)
$$

Equation (Eq. 4.11) is the conclusion of the Gaussian distribution in a discrete and continuum media. In a discrete space the distribution of monomers is the product of all the discrete probabilities. In continum media when we know the partial distribution of monomers, we can integrate the total distribution of the chain. Therefore, for a long chain we can approximate the Gaussian distribution with continuum limit. The details of which we will discuss later. Now from a normalization prospect we have: 


$$
\prod_{n=0}^{N} \exp \left(-\frac{3}{2 b} \sum_{n=1}^{N}\left(\frac{\partial R}{\partial n}\right)^{2} \Delta n\right)=\int \delta R \exp \left(-\frac{3}{2 b} \int_{0}^{\mathrm{L}}\left(\frac{\partial R}{\partial l}\right)^{2} d l\right)=1
$$

\subsubsection{Discrete Space model}

The lattice or discrete model is known as the Bond Fluctuation Model (BFM)[107]. In the continuum model, the center of the beads could be anywhere in 3-dimensional space, while in the discrete model, the monomers are in predefined unit cells or grid surface, and bonds connect them together[108]. The discrete space is called a lattice and each lattice unit is called a site. In two-dimensional space, we can have a square lattice or triangular lattice space. In three dimensions we very often use cubic cells or sometimes diamond lattice. The number of neighbors of each lattice is called the coordination number. The coordination number for a square lattice or a diamond is four and for a triangular lattice or a cubic lattice it is six. In a cubic lattice, monomers can hop from one site to another, but they can only hop to the places where their new bonds to their nearest neighbors has a radius of $(\sqrt{1} l, \sqrt{2} l, \sqrt{3} l)$.

\subsection{Gaussian Distribution}

Using a Gaussian distribution, the length between monomers, the Kuhn length, l, determines the conformation properties of the polymer. Depends on polymer, if the Kuhn length is small, the conformation of the polymer follows the Brownian distribution and is quite flexible, whereas for a long Kuhn length polymer is quite stiff and not flexible[109],[110]. At high temperatures, the long polymer chain will be crumpled and the bond between two monomers tends to become zero. In these circumstances, the polymer will become flexible. Here in this thesis we are interested in high temperatures. In this thesis, the system in some parts is at temperatures high enough, that the bond length between two monomers will become quite small and the polymer tends to be flexible.

For flexible polymers, the bond vectors are totally uncorrelated, therefore their orientations are independent of each other. The correlation between bond vectors can be defined by: 


$$
\left\langle\tau_{i} \cdot \tau_{j}\right\rangle=l^{2} \delta_{i j}
$$

where $\delta_{i j}$ is kronecker delta. For a stiff polymer or a semi-flexible polymer, a worm-like chain model can be applied[57]. In this model, we need to consider that the intrinsic stiffness is proportional to the energy cost for bending polymer. The simplest bending Hamiltonian is like one-dimensional Heisenberg model for ferromagnets:

$$
H=-\epsilon \sum_{i=1}^{N-1} \vec{r}_{i+1} \cdot \vec{r}_{i}
$$

where $\epsilon$ is the friction coefficient. Where $r_{i}=l$. In the continuum limit noted by $N \longrightarrow \infty, \epsilon \longrightarrow \infty, l \longrightarrow 0$ and assuming $\epsilon / N=$ constant, the contour length will be also constant:

$$
-r_{i+1} \cdot r i=\frac{1}{2}\left[\left(r_{i}-r_{i+1}\right)^{2}-2 l^{2}\right]
$$

Then the Hamiltonian equation will be:

$$
H=\lim _{N, \epsilon \rightarrow \infty, l \rightarrow 0} \frac{\epsilon l}{2} \sum_{i=1}^{N-1} l\left(\frac{r_{i}-r_{i+1}}{l}\right)^{2}
$$

In the continuum limit, the tangent vector with the arc length $s$ wil be equal to:

$$
\frac{\partial r(s)}{\partial s}=\lim _{l \rightarrow 0}\left(\frac{r_{i}-r_{i+1}}{l}\right)
$$

by converting discrete space into continuous space $\sum_{i=1}^{N-1} l$ to $\int_{0}^{L} d s$ we find:

$$
H=\frac{\kappa}{2} \int_{0}^{L} d s\left(\frac{\partial r(s)}{\partial s}\right)^{2}=\frac{\kappa}{2} \int_{0}^{L} d s\left(\frac{\partial^{2} R(s)}{\partial s^{2}}\right)^{2}
$$


From Hamiltonian we can find the partition function of the system.

$$
Z=\int D\left[r_{s}\right] \delta r(s)-1 \exp (-\beta H[r(s)])
$$

By having the partition function, we can find the total conformation of the long polymer chain with length $\mathrm{L}$ and energy of the conformation $k_{B} T$.

\subsection{Ideal and real polymer chain distribution}

In reality, the interaction between monomers is such that two more monomers cannot occupy a single space even partially (Self-Avoiding Random Walk or SAW model)[111]. However, we often make an ideal assumption that monomers can overlap with each other. In a lattice model, this corresponds to monomers occupying the same site (Figure. 4.3). This leads to the definition that in ideal polymer chain model crossing over itself is allowed while in the real chain model (SAW) is not (Figure. 4.5).

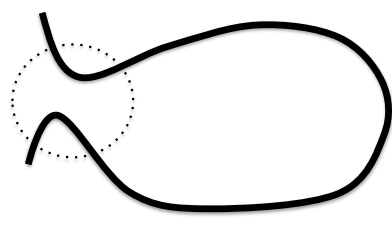

(a)

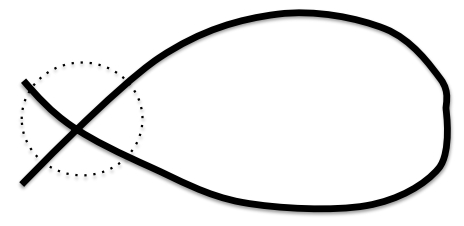

(b)

FiguRE 4.5: Figure (a) shows the real polymer chain, where the two monomers cannot be closer than a specific length and Figure (b) shows the ideal polymer chain where two monomers can cross over each other.

Both types of chain can be modeled as a random walk. For a real chain, two different segments can never meet each other at the same point; however, in the ideal chain they can. For an ideal polymer chain we apply Gaussian distribution model to describe the 
probability distribution of the chain; however, for a real polymer chain a non-Gaussian distribution model must be applied.

\subsubsection{Random Walk model of Ideal chain}

The random walk model was already discussed in a general way in chapter 2 . In this section, we discuss its role in the ideal polymer chain model, where each monomer plays a random walker role. Suppose a random walker starts at the origin point $x=0$ in one-dimensional space, where the size of each step is identical and equal to $l$. After each step, the random-walker loses its memory, a property that comes from the Markovian chain process[112]. If $\mathrm{n}$ step from the total $(N)$ was toward the right and the $N-n$ step was toward left, then the probability distribution by of having right steps $(p)$ and left steps $(q)$ is binomial and equal to:

$$
(p+q)^{n}=\sum_{n=0}^{N} p^{n} q^{N-n} C_{N}
$$

Since the probability of going left or right is equal to one, the total sum of $p+q$ will be equal to one. After $\mathrm{N}$ steps the probability of having $\mathrm{n}$ steps in the right direction will become:

$$
P_{n}=2^{-N} C_{N}=\frac{N !}{n !(N-n) !}
$$

Assuming there is equal probability of going left or right, if we set $p=1-q$, we have the identity:

$$
2^{N}=\sum_{n=0}^{N} \frac{N !}{n !(N-n) !}
$$


Thus, the expected value of $n$ is given by:

$$
\begin{aligned}
n P_{n}= & 2^{-N} \sum_{n=0}^{N} \frac{n N !}{n !(N-n) !}=2^{-N} N \sum_{n=0}^{N} \frac{(N-1) !}{(n-1) !(N-n) !} \\
& =2^{-N} N 2^{N-1}=N / 2,
\end{aligned}
$$

and the second moment is given by:

$$
\begin{aligned}
\left\langle n^{2}\right\rangle= & \sum_{n=0}^{N} n^{2} P_{n}=2^{-N} \sum_{n=0}^{N} \frac{n^{2}(N) !}{(n) !(N-n) !} \\
& =2^{-N}\left[N(N-1) \sum_{n=2}^{N} \frac{(N-2) !}{(n-2) !(N-n) !}+\sum_{n=1}^{N} \frac{(N-1) !}{(n-1) !(N-n) !}\right] \\
& =N(N+1) / 4
\end{aligned}
$$

Now let see the changes of $P_{n}$ when $N$ increase to the large number. In order to study $P_{n}$ (for discrete space model) or $P(x)$ (for a continuous space model). The general idea of normal distribution comes from one of the three fundamental equations of physics, diffusion equation. A particle starts from the origin and diffuses over time so that its position follows the Gaussian distribution [113]. In polymer physics, when the number of steps in random walk model becomes very large, we use the same approach in order to find the probability of polymer chain conformation applied to (Eq. 4.21). By substituting $\mathrm{n}$ for $\Delta s$ we can interpret the step motion of random walkers as a polymer chain conformation[114]. For large $N$ we use the Stirling approximation.

$$
\begin{aligned}
\ln P_{n}= & -N \ln 2+N(\ln N-1)+n(\ln (n-1))-(N-n)[\ln (N-n)-1] \\
& =-N \ln 2+N \ln N-n \ln n-(N-n) \ln (N-n)
\end{aligned}
$$

If we consider one dimension and start from the origin $\left(x_{0}=0\right)$ the arbitrary displacement after $\mathrm{n}$ step is $x_{n}=n b$, we can convert $n=x_{n} / b$ and rewrite $N-n$ to $\left(N+x_{n} / b\right) / 2$, in which $\mathrm{b}$ is Kuhn and $x_{n}$ is the displacement length; this equation will become: 


$$
\begin{aligned}
\ln P & \approx-N \ln 2+N \ln N-\frac{1}{2}\left(N-x_{n} / l\right) \ln \left[\left(N-x_{n} / l\right) / 2\right]= \\
& -\frac{1}{2}\left(N+x_{n} / l\right) \ln \left[\left(N+x_{n} / l\right) / 2\right]=N \ln N-\frac{1}{2}\left(N-x_{n} / l\right) \ln \left(N-x_{n} / l\right) \\
& -\frac{1}{2}\left(N+x_{n} / l\right) \ln \left(N+x_{n} / l\right)=-\frac{1}{2}\left[\left(1-\frac{x_{n}}{N l}\right) \ln \left(1-\frac{x_{n}}{N l}\right)+\left(1+\frac{x_{n}}{N l}\right) \ln \left(1+\frac{x_{n}}{N l}\right)\right] \\
& \approx-\frac{1}{2} N\left(\frac{x_{n}}{N l}\right)^{2}=-\frac{x_{n}^{2}}{2 N l^{2}} .
\end{aligned}
$$

By normalizing $\int p(x) d x=1$, we can find the probability that random walk be at position $x_{n}=x$ after $\mathrm{N}$ step:

$$
P(x)=\left(2 \pi N l^{2}\right)^{-1 / 2} \exp \left(-\frac{x^{2}}{2 N l^{2}}\right)
$$

Where we assume the length of displacement is very small that tend to be zero $x \rightarrow$ and polymer is continuously distributed.

\subsubsection{Distribution function of ideal polymer chain}

As we learned from section. 4.1.1, in the limit of $N \rightarrow \infty$, the ideal chain behaves as the continuum model. Since each part of the chain is identical we can take a cross section of the displacement $\left(x_{1} \rightarrow x_{2}\right)$ and find the probability density between these two points. To do this we can consider the midpoint between these two points and define $G\left(x_{1}, x_{2}, n\right)$ as a transition probability.

$$
G\left(x_{1}, x_{2}, n\right)=\left(2 \pi n l^{2}\right)^{-3 / 2} \exp \left(-\frac{3\left(x_{1}-x_{2}\right)^{2}}{2 n l^{2}}\right)
$$

We assume the number of segment of the polymer between these two points are n, where $n<<N$, then we call $G\left(x_{1}, x_{2}, n\right)$ is the transition probability for a point $\left(e . g . x_{2}\right)$ to move into area around $x_{1}$ in a small volume of $d x_{1}$ (with the total number of steps $=$ $\left.n_{1}\right)$. Consequently, we can join two groups of units with the inter-joint point $x^{\prime}$. If the number of units from $x_{1}$ to $x^{\prime}$ is $n_{1}$ and the number of units from $x^{\prime}$ to $x_{2}$ is $n_{2}$ then the probability that distribution occur through the displacement of the chain from $x_{1}$ to $x_{2}$ is given by[115]: 


$$
\int G\left(x_{1}, x^{\prime} ; n_{1}\right) G\left(x^{\prime}, x_{2} ; n_{2}\right) d x^{\prime}=G\left(x_{1}, x_{2} ; n\right)
$$

in which $n=n_{1}+n_{2}$. Unlike the random walk model for the ideal polymer chain, the Gaussian distribution can be defined for limited number of units even when $n \rightarrow 0$, then $x_{1}$ and $x_{2}$ are in the same place:

$$
G\left(x_{1}, x_{2}, 0\right)=\delta\left(x_{1}, x_{2}\right)
$$

This shows that Gaussian distribution of the ideal polymer chain can follow the normal distribution of the diffusion equation. Thus $G\left(r, r^{\prime} ; n\right)$ for three dimensions will satisfy:

$$
\left[\frac{\partial}{\partial n}-\left(b^{2} / 6\right) \nabla^{2}\right] G\left(r, r^{\prime} ; n\right)=\delta(n) \delta\left(r-r^{\prime}\right)
$$

\subsection{Excluded Volume Effect}

This is the property that distinguishes the real polymer chain model from the ideal chain model. We say sphere A is excluded by sphere $A^{\prime}$ when their distance is not less than the centre-to-centre distance of the spheres $d_{A A^{\prime}}$. If the excluded volume of one sphere changes from zero to $v_{e x}$ then the total available space for the neighbor will change from $V$ to $V-v_{e x}[116]$. Consequently, the configurational entropy will be changed as below:

$$
\Delta S=k_{B} \ln \frac{V-v_{e x}}{V}
$$

This equation shows the change of configurational entropy is directly related to the ratio of excluded volume over the volume of spheres. Also, this means that the Helmholtz free energy difference will be proportional to this ratio as well $\left(\Delta A / k_{B} T\right)$. The excluded volume effect can be present even in solution. If the segment unit length is $l$ then each monomer occupies a cube of volume $l^{3}$ and no other monomer can be found in that area. 
The excluded volume effect can be considered as an interaction and therefore the corresponding free energy per chain is $F / k_{B} T=\left(N^{2} / 2\right) v_{e x} / R^{3}$, which is a function of the scaling exponent $\nu\left(R \approx N l^{\nu}\right)$, where $\nu \approx 2$. The contribution of free energy in the total conformation energy $\left(\Delta U / k_{B} T\right)$, by assuming $l^{3}=v_{e x}$ will be[117]:

$$
\Delta U_{e x} / k_{B} T \cong l^{3} N^{2} / R^{3}
$$

At higher concentrations, the excluded volume effect can be considered between the pair of different chains. The thermodynamic properties of the polymeric system have been directly related to the effective volume of space. When this parameter becomes zero, it is called $\theta$ temperature. For $T<\theta, \nu$ will become negative and for $T>\theta, \nu$ (Flory exponent: see section 4.0.3) becomes positive. For $T=\theta$ self-avoiding random walk will transform into random walk, or in another words monomers can overlap with each other. Therefore monomer penetration influences polymer chain size. In a good solvent, the chain length is larger than a $\theta$ solvent, so the coils are denser and the radius of gyration is $2 / 3$ power of $\mathrm{N}$ instead of one.

\subsection{The Real Polymer Chains Model}

So far we have understood the concept of ideal chain and real chain, also the excluded volume effect that monomers cannot occupy the same place. The reason that we cover both states of the polymer chain in this thesis is because our system can vary from ideal chain to real chain due to the temperature. Therefore, we need to know how to model each state of the system.

\subsubsection{Self-Avoiding Random Walk Model (SAW)}

A polymer can be folded into the 3D complex structure in a solution[118]. The complex configuration depends on long range and short range interactions of the polymer. For example, the force between monomers in a chain, and their interactions with the solution, can determine the total number or the ultimate form of the configurations of the folded polymer chain. 
If we want to model a real polymer chain in $3 \mathrm{D}$, the simple random walk model is not a suitable one[119]. We need a dynamical model that responds to all impulsive and attractive forces of the polymer in a solution. From the mathematical point of view, this is not a Markov process, because the thermodynamic properties of the system are highly related to the properties of the solution and chain. At very high temperatures monomers can be very close to each other as much as they can overlap, and we can model them as an ideal chain. While at lower temperatures monomers cannot be closer than their diameters (excluded volume effect); a model that uses the latter approach is called the self-avoiding random walk.

The displacement $x$ and the variance in the displacement of the self-avoiding random walk model in one dimension is given by:

$$
x=n \propto t
$$

and,

$$
\sqrt{\left(x^{2}\right)}=\sqrt{n} * \sqrt{t}
$$

However, in 2D, their relation will become rescaled and depends on the Flory exponent $(\nu)$, which is directly related to the type of the solution (see section 4.0.3) and temperature of the medium. In our case, the Flory exponent is at the intermediate range.

$$
\frac{1}{2} \leq \nu<1
$$

In some self-avoiding random walk models the probabilities of available sites for displacing a random walker's place are equal. However, in some cases, each MC step has its own weight that depends on the availability of the sites. If monomers have been in a site once then that site will be rejected for the next move. So the number of discarded 
walks will increase exponentially.

$$
\frac{\text { instantaneous generated walk }}{\text { total attempted walks }}=e^{-c n} \text {, }
$$

where $c$ is an iterative constant. In this thesis, if the place is empty, the monomer can occupy it, no matter whether it has been occupied with a monomer before or not.

So far we have discussed the general approach of monomer displacement in a system. However, the performance of simulation of a polymer in a system is highly related to other physical conditions of the system for example, $\mathrm{pH}$, temperature, and solution molecules. In order to improve the performance of the simulation effectively, we need to apply effective conformation method in the simulation. For different system, we may need to apply different conformation approaches. In the next section, we will explain some common methods that can increase the rate of simulation significantly.

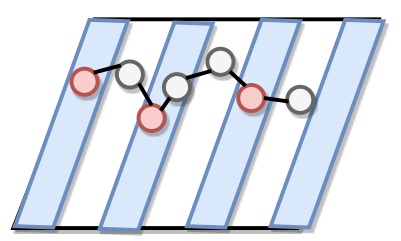

FiguRE 4.6: This figure shows a surface with the striped pattern of sticky patches. The monomer can adsorb on those patches. Blue stripes show the sticky sites. Once a couple of monomers adsorb on blue stripes the movement of monomer become more restricted. By applying right conformation method we can improve the simulation performance.

- Reptation method: this method was first introduced by Pierre-Gilles de Gennes, from part of his work on liquid crystals and polymers [120]. According to the de Gennes assumption, any polymer can be confined in a tube. Any thermal fluctuation can cause the polymer to reptate as a result of polymer entanglement and being analogous to slithering in a tube. This is one of the most common ways of generating SAW in a medium. It performs very well for dilute media with the short-time limit. Basically, we choose either end of the chain and choose one of the available direction randomly and move the monomer to this place. However for the long time limit is not efficient. In addition, this method is sensitive to its initial state. This method is not suitable for attractive sites (see figure 4.6); it becomes very slow if either ends become adsorbed on attractive sites. Therefore, 
have not applied this approach to our system. Figure 4.7.a shows the reptation method in lattice space.

- Kink-Jump:, this method is suitable for the system with a concentrated solution, when the polymer chain hits the molecules of the solution. In this numerical method we apply bond fluctuation model (BFM); monomers can only fluctuate into certain places. The restriction of the movement is because of the bonding between monomers, they can change their places as long as they do not break their bonds with their neighbors. In part of our local moves we use this approach and we combine it with another approach. In the Kink jump method, since we can choose different monomers on a chain, the system will not be stopped from evolution after a few monomers become adsorbed on sticky patches. Figure 4.7.c shows the Kink-Jump method in lattice space.

- Pivot algorithm:, In this method, we do not have only single steps but also, we have two or more sub-walks which can be due to the rotational or transformational symmetry. This method is efficient for non-uniform media, but, for uniform media is probably not the right method. As we mentioned before, we are interested in, simulating the polymer chain adsorption on the surface. We want to consider some attractive sites on the surface, each of which have a specific pattern. Figure 4.6 shows one of these patterns, which is striped. We will test the Pivot algorithm approach for such a system in Chapter 5 , but we will see due to the overlap energy system cannot fluctuate properly based on BFM rule and the presence of sticky sites. Figure 4.7.c shows the Pivot algorithm method in lattice space.

\subsection{Statistical Mechanics of a Polymer Chain in a Solution}

From a statistical mechanical point of view, calculating the partition function of polymeric systems enable one to determine the equilibrium configuration of a polymer chain in a solution. There are some analytical methods for finding these statistical functions, with some methods giving exact solutions while others result in approximate solutions. For non-interacting systems calculating the partition function of the system is not difficult; however, these systems are not very relevant. Typically we are interested in interacting systems. The main difficulty in finding exact solutions for such systems are 


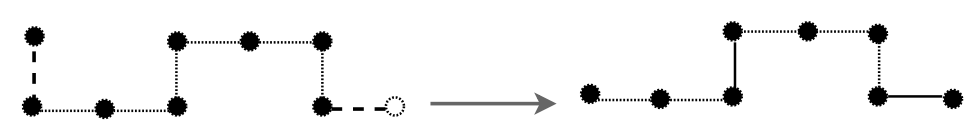

(a)
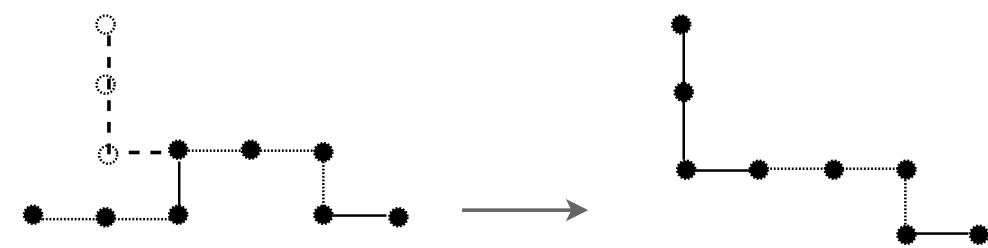

(b)
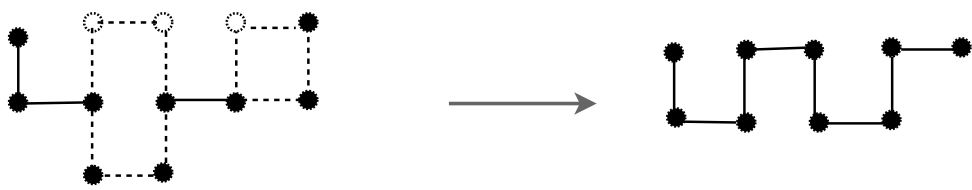

(c)

Figure 4.7: These figures show the changing places of the monomers. Dash circles are the new places of the monomers and dash lines show the breaking and making new bonds. The new chain structures show on the right side of picture. Figure (a) shows Reptation algorithm where one end of the polymer chain is moved to the other end. Figure (b) shows the first monomer of the displaced part is rotated 90 degrees to the new place with the rest of the chain. Figure (c) shows two different types of kink-jump displacement, which are all in the range of the BFM length.

the fluctuations of the polymer configuration. There are some methods for solving such systems such as mean field theory, series expansion, and renormalization group methods. In this thesis, we use the series of expansion approach and apply this to polymer chains at surfaces[121].

\subsubsection{Series Expansion}

This technique uses a perturbation approach, where the partition function is modeled as a series of a certain parameter in different subensembles of the system. This parameter can be the temperature which we expand from 0 to $\beta^{-1}\left(\beta=\left(k_{B} T\right)^{-1}\right)$ or even for some system series with very low densities $\rho=0$ to $\rho$. Applying series expansion helps us to find the phase transition of the systems in the region where their convergence tends to be very slow. The next step is how to calculate the partition function, which corresponds to all of the configuration space for these sub-ensembles. Simply summing the partition function over all configurational phase space is not feasible, because by increasing the size 
of the system, the total number of configuration will increase exponentially. For example, in three dimensions with 10 lattice sites in each axis and 10 particles, the total number of discrete configurations will be $\left(10^{3}\right)^{10}$. Obviously, 10 particles is a very small number and for a more real system, we need more particles. The simplest way of computing configurational space is to select a random number of configurations. This approach in the long time limit leads to an equivalent number of occurrence of microstates and the same value of their probability distributions. If we select $N_{c}$ number of configurations randomly, then the average the partition function will be:

$$
Z=\bar{z}=\frac{\Omega_{\mathrm{total}}}{N_{C}} \sum_{i=1}^{N_{c}} \exp \left(-\beta E\left(\sigma_{i}\right)\right.
$$

where $\Omega_{\text {total }}$ is the total number of microstates, $\sigma$ is ith configuration and $Z$ is the partition function. The terms in this equation are dominated by those at high temperatures and energies. The thing that we need is to make a balance between microstates through bias algorithm. Suppose that the probability of each microstate is $p\left(\sigma_{i}\right)$. At the first moment a natural choice is the Boltzmann distribution:

$$
p\left(\sigma_{i}\right)=-\frac{\exp \left(\beta E\left(\sigma_{i}\right)\right)}{Z}
$$

where $E\left(\sigma_{i}\right)$ is the energy of configuration $i$. Since $Z$ is sum over all possible configurations and calculating the probability of occurrence of each microstate from total configuration space is not an easy task, we calculate the transition probability from configuration i to configuration j. $\rho_{i j}=P\left(\sigma_{i} \Rightarrow \sigma_{j}\right)$ is the density matrix that gives the transition probability between the nearest neighbors of subensembles. Here, each element of density matrix only depends on states $i$ and $j$. The next step is to connect $\rho_{i j}$ to the probability of each microstate. The probability of microstate i at time, $t=1$, is given by:

$$
p_{i}(1)=\sum p_{i}(0) \rho_{i j}
$$

Now we need to produce the density matrix. The value of $\rho_{i j}$ can be determined by 
scattering matrix $R_{i j}$. The matrix $R_{i j}$ tells us what states are available for transition. In practice we can implement this algorithm by selecting a trial move from $\mathrm{i}$ to $\mathrm{j}$ and producing a random number between $[0,1]$. If the value of $R_{i j} \geq 1$ the move is accepted, otherwise the acceptance has a probability. $R_{i j}$ matrix is an arbitrary symmetric matrix:

$$
\rho_{i j}=\left\{\begin{array}{l}
R_{i j}, \quad U_{j}<U_{i} \\
R_{i j} \exp \left[-\beta\left(U_{j}-U_{i}\right)\right] \quad \text { otherwise }
\end{array}\right.
$$

In the following section, we will show how we can use this approach in order to find the free energy of the system[122].

\subsubsection{Umbrella sampling}

Umbrella sampling is a method for free energy calculation in terms of the reaction coordinates, which was developed by Torrie and Valleau in 1977. The method uses a bias potential, which is applied from one state to another. If we consider a molecule that its center of mass is negligible at a distance $z$ from interactive surface, the probability distribution of the configuration of the molecule will become[123]:

$$
\rho(z) \propto \int \exp ^{-\beta U\left(\sigma_{N}\right)} \delta\left[z-z\left(\sigma_{N}\right)\right] d\left(\sigma_{N}\right) \propto \exp ^{-\beta F(z)}
$$

Figure 4.8 shows a schematic probability distribution along the $\mathrm{z}$ axis which is the reaction coordinate. The height of the free energy barrier tells us that there is a small probability that a molecule can transit from its current state to other states. For larger heights, this causes a problem. By applying bias sampling we can only consider the model through subensembles of $\mathrm{z}$ near $\mathrm{z}_{j}$. This means that the partition function will be restricted to distances near the surface of the reaction discarding the rest of the configurational space[124]. The most common way of biasing the configuration is as follows:

$$
-\beta U_{j}\left(\sigma_{j}\right)=-\beta U_{i}\left(\sigma_{i}\right)+\eta_{i j}(z)
$$




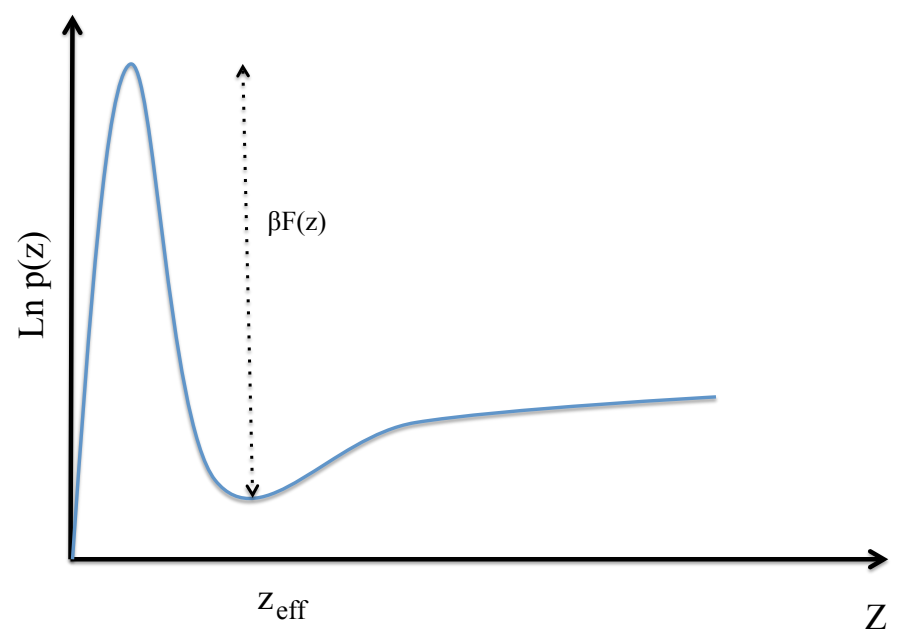

FiguRE 4.8: Free energy barriers of transition from z to z'.

where:

$$
\eta_{i j}(z)=\beta k / 2\left(z_{i}-z_{j}\right)
$$

is a harmonic potential. The effective weighting potential is given by:

$$
-U_{j}\left(\sigma_{j}\right)=-U_{i}\left(\sigma_{i}\right)+k / 2\left(z_{i}-z_{j}\right)
$$

The choice of force constant in equation ( 4.44) is very important. If the force constant is too small, it will cause less effective biasing. If it is too large this can lead to a weak overlap between z distribution and limited numbers of occurrence. In each simulation step we can find the unweighted probability distribution by:

$$
p\left(\sigma_{N}\right) \propto p_{j}\left(\sigma_{j}\right) e^{-\eta_{j}(z)}
$$

One of the advantages of umbrella sampling is that it can be applied both in MC and MD simulation. The umbrella method, after iterative reweighting, is a good method to find the free energy[125]. The method can also be applied to canonically average parameters. 
One of the disadvantages of this method is the difficulty of finding a suitable sampling distribution. This can cause some issues for large systems[126].

\subsubsection{Expanded ensemble method}

This method was developed by Lyubarstev et al. It is similar to umbrella sampling in that it uses expansion; however, in umbrella sampling, we compute the mean field potentials along the reaction coordinates, whereas in the expanded ensemble method we sample from a distribution of random-walkers[127]. The expanded ensemble method (EEM) was first applied to charged hard spheres, where these hard spheres were able to transit between a range of different replica temperatures $(\beta=1 / k T)[128]$. A replica temperature is allocated to each subensemble while other physical and thermodynamic conditions of subensembles are identical. Therefore, the partition function for the test of subensembles is given by:

$$
Z=\sum_{j} z_{j} \exp \left(\eta_{j}\right)
$$

where $\eta_{j}$ is a bias potential and it is an iterative function. $z_{j}$ is the partition function of each sub-ensemble at each temperature $\left(\beta_{j}^{-1}\right)$.

$$
Z=\sum_{\sigma} \exp \left[-\beta_{j} E(\sigma)\right]=\exp \left(-\beta_{j} F_{j}\right)
$$

The equation ( 4.47) gives us the total partition function of the system in a different range of temperatures. Now we need a transition matrix to prescribe the transition between neighbouring subensembles. The bias probability from state $i$ to $j$ is given by:

$$
\Delta \beta=\left(\beta_{i}-\beta_{j}\right) U(\sigma)+\eta_{i}-\eta_{j}
$$

where $\sigma$ is the particular configuration of space. All the subensembles start from the same configuration but evolve independently between transitions. From Eq( 4.46) we can find the contribution of each subensembles in the total probability. 


$$
P_{j}=z_{j} \exp \left(\eta_{j}\right) / Z
$$

We start every state from the initial state that $\beta=0$ or very high temperature since we assume the empirical coefficient at that temperature is $\eta=0$. We can use the partition function of the ideal gas in three dimensions simple cubic lattice $Z=6^{N}$, where $\mathrm{N}$ is the number of monomers. As a consequence, the free energy, which is proportional to $k_{B} T \ln (Z)$ is obtainable. If we assume the probability of occurrence of subensemble at $\beta_{0}=P_{0}$ and the probability of occurrence of any arbitrary subensemble is $P_{j}$ then the ratio of these two probabilities that assign the probability of transition from one subensemble (in this case $\beta=0$ ) to another $\left(\beta_{i}\right)$ is given by:

$$
\frac{p_{i}}{p_{i}}=\frac{z_{j} \exp \left(\eta_{j}\right)}{z_{0} \exp \left(\eta_{0}\right)}=\exp \left(-\left(\beta_{j} F_{j}-\beta_{0} F_{0}\right)-\eta_{i}-\eta_{j}\right)
$$

Then we can find the free energy difference between two subensemble.

$$
\beta_{i} F_{i}-\beta_{j} F_{j}=\ln \frac{p_{i}}{p_{i}}+\eta_{i}-\eta_{j}
$$

However, we need to choose optimal accurate $\eta_{i, j}$. We start with the initial value of $\eta_{i}^{0}$ :

$$
\eta_{m}^{0}-\eta_{m-1}^{0}=\left(\beta_{m}-\beta_{m-1}\right) U_{i j}
$$

where $m$ is the particular subensemble which its nearest neighbours are $m \pm 1$. Here we consider left nearest neighbour $(m-1)$. However finding the optimal $\eta_{m}$ iteratively leads to self-consistent solution:

$$
\eta_{m}^{i}=\eta_{m}^{i-1}-\ln \left(p_{m}^{i} / p_{m}^{0}\right)
$$


In an equilibrium state, equation $\operatorname{Eq}(4.51)$ will become $\eta_{m}=\beta_{m} F_{m}$ because $p_{j} \neq p_{k}$, thefore, $B_{i} F_{i}-B_{j} F_{j}=\eta_{i}-\eta j$ and from equation $\mathrm{Eq}(4.52)$, we have:

$$
U(\beta)=\frac{\partial(\beta F(\beta))}{\partial \beta} .
$$

Although in this description of the method, the temperature was changing in different subensembles, we can apply this method for different chemical potentials and variations in the number of particles.

One of the applications of expanded ensemble methods is protein folding which was modeled by a polymer chain whose temperature is used in a certain range to examine its configuration[129]. Spin glass theory which was modeled by three-dimensional Ising model is the best example of sampling theory for the expanded ensemble method [130]. This was the simulation that proved that replica symmetry can break the theory.

Generally, the EEM method provides us this opportunity to explore the configuration of space and helps us to find the free energy difference through measuring occurrence.

\subsection{Conclusion}

This chapter is an introduction to polymer physics. Some subjects such as scaling, coarse graining, and random walk models are the key elements in the simulation of polymers in physics. In this chapter, we have explained some common numerical methods that can be used in a polymeric system. Some of these methods can be applied for polymer adsorption on the surface. Simulating the polymer adsorption on the surface is a complex process. There are a different range of interactions near the surface: short-range interactions such as monomer-surface interactions and long- range interactions such as monomer-monomer interactions. Each type of interaction has its own time interval. We used Kinetic Monte Carlo simulation to cover different time intervals that a system needs in order to have the related evolution. In the next chapter, we will explain about the system that has both attractive and neutral sites. Once a few monomers become adsorbed on the surface, general KMC simulation will become very slow. We employ some conformation techniques such as the kink-jump method which we have discussed from here, in order to increase the time of evolution in the system. 



\section{Chapter 5}

\section{Homopolymer Adsorption onto}

\section{Heterogeneous Surfaces}

The adsorption of polymer chains on a solid surface is important in biology, tribology, and industrial processes. Lubrication, DNA packing, chromatography and the synthesis of artificial membranes all involve polymer adsorption onto a surface[131],[132],[95]. Polymers on a surface effectively give rise to new materials with potentially useful properties[21],[133]. To understand this, we need to study the nature of the applied polymer chain and its environment to determine the macroscopic properties of the interface[134]. As an example, thermodynamic properties such as the temperature of the polymeric system have been directly related to the effective volume around the monomers $(\nu)$, the space which monomers exclude other monomers. Factors such as solvent quality, the size of macromolecules, end-to-end vectors, and radius of gyration length have been extensively explored at the molecular level over the last four decades[135].

However, in this thesis, we are interested in the dynamics of the polymer solution in the vicinity of the liquid-solid surface[136]. In general, the theory of the polymer-solid interaction is very complex [137]. This will become more complicated when we consider corrugated crystal structures with different kinds of interactions and surface energies.

In practice most of the surfaces are inherently heterogeneous, so the study of the statistical and dynamical behavior of polymers near a heterogeneous surface is crucial. From a thermodynamic point of view, polymers will minimize their energy by maximizing the number of adsorbed monomer-sites, but this leads to entropy loss, due to the 
reduction of free available monomer for building a new configuration. In this chapter, we will investigate the behavior of homopolymer chain on complex surfaces using Monte Carlo methods[138]. The surface structure that we study consists of both interacting and non-interacting regions. From a surface structure point of view, there are some attractive regions on the surface with binding energies that can overcome the loss of entropy of an adsorbed polymer, whereas in other areas the entropy loss of the polymer chain is greater than the binding energy, so a depletion layer will be developed. This balance of energy for the short chain is less than that for the long chain. As a consequence, short chain polymers bind more easily to a surface.

The contribution of entropy to free energy is proportional to the temperature. At high temperature, entropy will dominate, while at low temperature, the adsorption energy will dominate. Our goal is to compute the critical value of the temperature $T_{c}$ of which both adsorption and desorption of the chain occurs with the same probability. Once the polymer chain fully recognizes the attractive pattern for adsorption (fully recognition of pattern by polymers means, when the polymer can self-assemble itself based on the pattern), another phase of the system will occur.

In this thesis, we studied a few periodic and non-periodic patterns of attractive sites. Not only is the temperature an important factor in phase transition, the length of attractive sites also plays a key role in the time of occurrence of the phase transition.

We start from attractive sites patterned on the length scale of a monomer and extend until the surface is effectively and homogeneously attractive. From this, we can consider the results from the nanostructured heterogeneous surface with uniform attractive surface continuously. We calculate the free energy of such a system with the expanded ensemble method. This method has been widely described in Chapter 4. This method has been studied for homogenous surfaces very well[139], however, the heterogeneous surface is a subject that have been discussed less especially in polymer physics. In this thesis, we are interested in heterogeneous attractive surfaces.

\subsection{Applications of Polymer Adsorption}

The polymer chain can be used to control crystal growth. When there is more than one type of energy of facets in a crystal structure, the polymer chain can adsorb on the a 
particular facet and help other facets to grow faster. One of the uses of the homopolymer adsorption on a solid substrate is in gas detection by semiconductors. Coating semiconductor substrates with monodispersed polymer is one method for increasing the performance of semiconductors or metals in detection of gases such as hydrogen[140],[141]. Polymers are highly sensitive and responsive to the environment via their configurations and entropy and are well-known as a smart material[142]. Another application is in optics and biosensors, where polymers can be patterned to different specific wavelengths of light[143].

Recently the sensitivity property of polymer has been shifted to a new era of applications, which is in the biomedical field[144]. Polymer chains have been employed to increase the efficiency of injection and drugs in terms of speed and coverage. Basically, drug molecule will attach to a polymer chain and that helps the drug to identify the target cells faster and more efficiently than with the regular approach[145].

The obstacle for all applications is the size of dispersion[146]. In nanostructure format, polymer chains are very stable due to their covalent bonds. The covalent bonds under thermal conditions become very weak and unstable. Therefore it is quite hard for the system to reach a stable state. As a result, studying the polymer adsorption as a function of temperature and environment is a crucial challenge for many chemical and biological processes[147].

\subsection{An Overview of Polymer Adsorption}

In 1953, the first discovery of the change in conformation of a polymer chain in the presence of a reflecting wall was made by Simha, Frisch and Eirich[148]. They studied the properties of a single semiflexible chain tethered to a planar surface with a longrange attractive potential by means of Monte Carlo simulations. They employed the bond fluctuation lattice model and the Wang-Landau sampling technique to find the adsorption isotherm[139]. Their discovery led to finding the thickness of the adsorbed layer of monomers at a $\theta$ point which was proportional to the square root of the molecular weight of the polymer. However, in 1960 DiMarzio et al. proved that the total number of distinct conformations had been overestimated[149]. They applied a new method for conformation of a polymer chain in a periodic cubic box. One monomer will change its 
place; however, chains never take backward steps, but rather always press forward. The polymer chain continues its displacement until their last monomer hits the other side of the wall of the simulation box. They showed that the evaluation of the conformation of the polymer chain must be considered one step before hitting the wall. Their calculation was consistent with their experimental result. Also, there were some diverse studies regarding the thermodynamic interaction parameters, applying scaling theory of polymer adsorption and finding the thickness of the layer of adsorbed segments around that time[150].

From the experimental point of view until 1955 only the total number of adsorbed monomers per site could be measured. Then after that, the thickness of the layer of adsorbed monomer could also be found.

From the theoretical point of view, statistical methods such as mean field theory, scaling theory, and the renormalization group have been developed to explain the computer simulations. Flory introduced the minimization of the free energy of polymer adsorption onto a surface in the lattice space, which combines the mean field theory and lattice models of polymer chains[151],[95]. In most of these studies, a flat surface was considered, although in reality most of the surfaces have some roughness, impurities, and defects. Magrather, Baumgfirtner and Muthukumar used MC simulation method of simple cubic $(S C)$ lattice[152]. Later on in 2002 Sharma et al.[153] and another group Tavakoli et al.[154]. in 2012 paid attention to dipole surface and holes on the surface. They applied a stripe pattern for such a heterogeneous surface.

\subsection{Theory of Polymer Adsorption}

Adsorption of small molecules has been discussed in Chapter 2 and 3. Unlike small molecules that have a well-defined structure, polymer molecules can adapt a complex variety of configurations and are prone to deform under small forces. Changing their shape under different circumstances influences their physical properties. They can be adsorbed in high amounts when the adsorption energy of the surface is greater than entropy loss of molecules leaving the solution. In earlier chapters, we studied a dilute solution, when a few molecules are adsorbed and the surface is occupied during the 
interaction. Once the interface becomes occupied, adsorption will gradually decrease, behaviour that is described by the Langmuir model.

Understanding the physical behavior of the polymer at an interface is much less well understood. The free energy of the polymeric system has a key role in determining the conformational transitions of molecules in a system and reveals the balance between entropy and adsorption energy that controls adsorption. The first person to discuss the importance of this was Flory[155]. He used the free energy minimization approach for a single chain in a solution, where its degree of scaling $\nu$ (see section 4.0.3) corresponds to the density of monomer with end-to-end vector length $(R)$. For the ideal chain this $\nu$ is 1 but based on the solvent, it can also be greater or less than one. Since the concentration of the monomers is constant, the only thing that determines this value is the strength of swelling and compacting of the polymer in a solvent. He assumed first the interaction of polymer is such that the ideal chain is stretched from both ends. Later this length of stretched chain is applied as a length scale to find the elasticity and the radius of gyration. In 1962 Silbereg showed that in order to calculate the free energy of the system near the interface it is important to consider solution loops and adsorbed train in different states[96]. He also emphasized that, although the scaling length $R^{2}$ at the $\theta$ point is proportional to molecular weight, for the adsorbed chain this relationship could be weaker than this. He proved the scaling properties of the polymer in a solution can be different from adsorbed chain, considering the segment interaction between chains. We do not use this here since we only consider a single chain.

The common goal of all these methods is to relate the conformational properties of the polymer to the stiffness of the polymer and its adsorption energy on the surface. This is also the approach we take here. We need the total potential energy in order to compute the partition function of the system and to calculate the free energy. The total energy of the system can be given by:

$$
U_{\text {total }}=U_{\text {steric }}+U_{B}+U_{A}
$$

This potential energy for our system consists of stiffness $\left(U_{B}\right)$, steric $\left(U_{\text {steric }}\right)$ and adsorption energy $\left(U_{A}\right)$. The stiffness of a polymer can be defined by bonded potential 
energy, which can be gdescribed by:

$$
U_{B}=\sum_{\theta} \kappa(1+\cos \theta)^{2}
$$

where $\theta$ is the average interactive angle between two segments. If the polymer chain represents different monomers, $\kappa$, which correspond to the stiffness of the polymer chain, it can vary from one type to another. However, in our simulations, we assume the monomers are identical and the bond length is small. We have learned from chapter 4 that for simplicity we consider the bond length between two monomers to be very small (the Kuhn length), so the polymer chain is very flexible and the bond between any three consecutive sites can vary from 0 to 180 . Therefore, from above equation the average $U_{B}$ in our case is zero.

The interaction between spherically-symmetric monomers can be defined by steric potential:

$$
U_{\text {steric }}=\sum_{i j} U\left(r_{i j}\right)
$$

where $r_{i j}$ is the distance between two interacting monomers, and $U\left(r_{i j}\right)$ is given by:

$$
U\left(r_{i j}\right)=\left\{\begin{array}{l}
k_{B} T \quad\left(r_{i}-r_{j}\right)<\sigma, \\
0 \quad\left(r_{i}-r_{j}\right)>\sigma
\end{array}\right.
$$

where $(\sigma)$ is the monomer diameter. The value of $U\left(r_{i j}\right)$ can be computed by the linkcell method[156]. At very high temperatures this steric potential is very large and is equivalent to the number of overlaps of monomer together on the same site.

The other potential energy which is important is the adsorption potential. We define this potential as

$$
U_{A}=N_{s} \epsilon
$$

$N_{s}$ is the number of adsorbed monomers and $\epsilon$ is the strength of adsorption. In our simulation the strength of adsorption is varried from 0 to $k_{B} T$.

In the following section we consider a polymer chain with 24 identical monomers that 
connected together; their interactions are covalent and are such that their nearest neighbors can interact with each other. However, this interaction is always much greater than adsorption energy of attractive sites such that they will never become detached from each other. This assumption is important at low temperatures due to the self-avoiding random walk model when monomers cannot cross over each other.

\subsection{Polymer adsorption near heterogeneous surfaces by MC method}

In this chapter, our aim is to simulate the adsorption of the polymer chain onto heterogeneous attractive sites[157]. We build a three-dimensional lattice (3D) box with a size of $D * D * D$ where $(D=25)$, and the cell length (L) is a monomer diameter. We neglect the solvent-monomers interactions and also solvent-solvent interactions. The heterogeneous surface is placed at $z=0$ and consists of neutral and attractive sites [158], whereas on top of the $3 \mathrm{D}$ simulation box at $z=D$, there is an impenetrable surface.

Here we compute the free energy difference by the KMC method. Finding the free energy by the KMC method is difficult due to the absence of function of configurational phase space to be averaged over the area of the surface [159]. Therefore we use a series expansion, in particular the expanded ensemble method that was introduced in the previous chapter in order to find the functional relation between phase space and free energy[160]. In addition, we are interested in finding physical quantities such as the center of mass (radius of gyration), the end to end vector, the absorption fraction and the probability of desorption. Furthermore, the most important part is to draw a phase diagram for the system based on the temperature and the size of attractive sites for different patterned surfaces.

\subsubsection{Free energy calculation of homopolymer chain on lattice space}

Calculation of the free energy or partition function is very useful for finding the phase transitions, equilibrium states, and conformational transitions [161]. Computationally both molecular dynamics and Monte Carlo methods can be used to calculate the internal properties of the system [162]. Methods such as umbrella sampling or series 
expansion can be used to simulate polymer adsorption. However, all of these methods are computationally demanding. Here in this thesis, we use an approach that is called the extended ensemble method as discussed in Chapter 4, which is quite efficient and accurate [163]. This method has been tested before in many systems such as Lennard-Jones fluids[164], ordered polyelectrolyte[165], quantum Heisenberg[166], and primitive electrolyte model[167]. In the present study, we apply this method on homopolymer adsorption in lattice space [168]. First, we assume our model is governed by the self-avoiding random walk on a lattice. We start by calculating the total number of configurations of the system at high temperature and for canonical ensemble system, where the temperature is fixed:

$$
S=-(\beta F)=\ln (Z)
$$

where $\beta$ is reciprocal temperature $\left(1 / k_{B} T\right), S$ is the entropy of the system and $Z$ is the partition function that is related to the configurational phase space of the system $\left(Z=\frac{1}{N !} \int \prod d q \exp (-\beta H(q))\right)$. When the contour length of the chain is quite short, counting the total number of configurations is quite straightforward. However, for a long chain, one can construct a random chain and then the fraction of nonoverlapping configurations over a total number of configurations gives us the partition function of the system, but again when the polymer chain becomes too large this is not an effective method. Instead in this study, we make a bridge between an impenetrable to a semipenetrable chain. Indeed, this method will cover different phases of the system that can occur over a certain range of temperature: for example, the excluded volume effect where overlaps are not allowed at low temperature and the ideal chain model where overlaps are allowed at high temperature. In order to use the expanded ensemble method for our system, we first construct the potential energy of the current conditions of the system. The semi-penetrable assumption means that monomers can overlap with each other with an energy overlap of $\epsilon_{m m}$. Also, segments can adsorb on the surface with an energy of absorption is $\epsilon_{m s}$. We have argued in section 5.3 that the total potential energy of the system is given by:

$$
U\left(q_{i}\right)=N_{v} \epsilon_{m m}-N_{s} \epsilon_{m s}
$$


where $N_{v}$ is the total number of overlapping monomers and $N_{s}$ is the total number of adsorbed monomers on the surface. The first term shows the steric potential and the second term shows the adsorption energy of the system. This equation is only for one conformation at a state i $\left(q_{i}\right)$. Consequently, non-dimensional Hamiltonian of the system is given by:

$$
H\left(q_{i}\right)=\beta U\left(q_{i}\right)
$$

where $U\left(q_{i}\right)$ is the potential energy of conformation. Since the temperature is the controlling factor in adsorption transition of the system we consider a set of subsystems (subensemble) over a range of temperatures such that each sub-system is at a certain constant temperature [169].

However, we know that many important phases are missing at high temperatures that can only be found in exploring the system at that range of temperature. As a consequence, using the expanded ensemble method (EEM), we not only consider each sub-ensemble at a constant temperature but we also examine the whole system at a range of temperatures. The canonical partition function will be the sum of all the configurations that we obtain from Monte Carlo averaging. We start by computing the partition function of each subensemble $\left(Z_{m}\right)$ :

$$
Z_{m}=\sum_{i} \exp \left(H_{m}\left(q_{i}\right)\right)
$$

where $H_{m}$ is the Hamiltonian of each subensemble at some temperature $\beta_{m}^{-1}$. The total partition function for $m$ subensembles using (Eq. 4.46) is given by:

$$
Z=\sum_{m} Z_{m} \exp \left(\eta_{m}\right)
$$

where $\eta_{m}$ is the weight function that balances between different subensembles to ensure that the simulation explores low energy states as well. From the previous chapter, we start giving an arbitrary value of zero to $\eta_{0}$ and finding the rest of the $\eta_{m}$ by the probabilities of their occurrences and we improve the values after several MC steps. 
In order to integrate all the subensembles in a simulation, we need to consider an extended system that contains all the subensembles. The subsystems are identical, except their temperatures $\beta_{m}^{-1}$ are different. The extended system needs to exchange between each subensemble in order to find the equilibrium state. We divide the simulation process into two steps: 1)-change the conformation of the chain in one subsystem, 2)-swap the sub-ensemble for transition from one temperature to another.

We consider these two steps independently below.

\subsubsection{Change the conformation of the chain}

In this step, the system is at a certain temperature in a subensemble and the only change is in the configuration of the system. Therefore the subsystem will have transition from one configurational state to another state within the subensemble. The probability of transition between state $i$ and $j$ is given by:

$$
w_{i j}\left(\beta_{m}=\text { constant }, q_{i} \rightarrow q_{j}\right)=\min \left\{1, \exp \left[-\beta_{m}\left(U\left(q_{i}\right)-U\left(q_{j}\right)\right)\right]\right\}
$$

After considering all the conditions for displacing monomers, we test the Rosenbluth condition, which measures the energy of the system after and before the transition, computing the energy of the new state and the energy of the current state[170]. where $\exp \left[-\beta_{m}\left(U\left(q_{i}\right)-U\left(q_{j}\right)\right)\right]$ is the result of (Eq 4.52). The transition will be approved with the probability $\min \left\{1, \exp \beta\left(U\left(q_{f}\right)-U\left(q_{i}\right)\right)\right\}$, otherwise the transition will be rejected. In Monte Carlo simulation algorithm such as metropolis or kinetic Monte Carlo, moves will be accepted if $\exp \left(\Delta U / k_{B} T\right)<r$ where $0<r<1$ is a random number. We applied the same method for the change of conformation and free energy calculation as well. There are different types of local moves of polymer conformation approaches, such as kink-jump, crankshaft, and reptation, which we already discussed in section. 4.5.1[171]. Applying each method has its own advantages and disadvantages, which are not the same for all types of systems. Selecting best method is based on their performances in a specific system, as well as their speeds, and efficiencies. For example the reptation method is not a suitable case for non-homogenous surfaces and it is mostly used for uniform media. 
The method that we apply is the combination of the single move MC (SMMC)[172], and configurational biased method (CBMC)[173]. It consists of three steps: select a monomer from a single chain randomly, then examine the total available sites in the radius of cubic cells (Figure. 5.1) $(\sqrt{1}, \sqrt{2}, \sqrt{3})$ and finally we select one of the available sites randomly. Secondly, check whether the nearest neighbors of the selected monomer are capable of changing their place in the newly translated position or not. Furthermore, we check whether one end of the polymer chain is close to the neighbours of selected monomer or not. If that is the case, the monomer can make a new bond with that end and the subsequent monomer becomes the last or first monomer of the chain and that end plays the role of a new neighbor for that monomer (Figure. 5.2). This method can dramatically increase the speed of evolution of systems with adsorption sites.

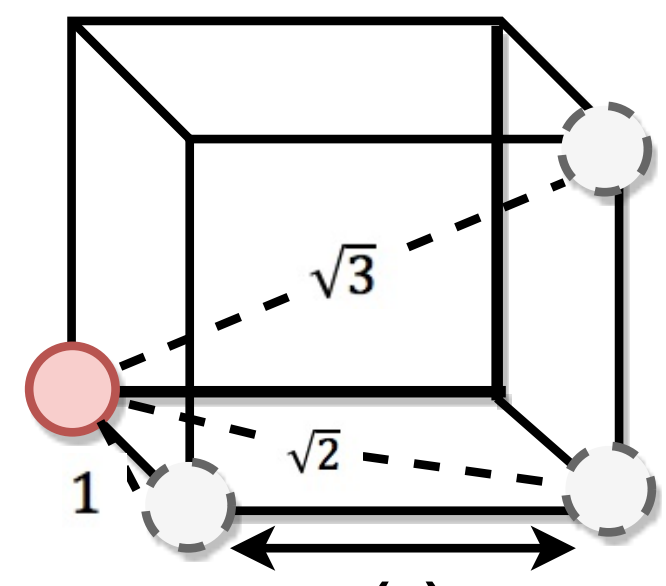

(a)

FiguRE 5.1: This figure shows the bond fluctuation of a polymer chain within a cube. The dash lines show the available places that one monomer can choose from. The red circle is the fixed monomer and dashed gray circles show the new possible position of monomer. Maximum displacement is a cube diagonal.

The situation is more complicated when some monomers are adsorbed on the surface, while their neighbors are free to make a local move. The system can transit from one conformation to another, if the strength of the surface is such that adsorbed monomer can not leave the adsorption sites then their neighbours can only move within a certain distance which is obeying the bond fluctuation model (BFM) assumption (section 4.4) 


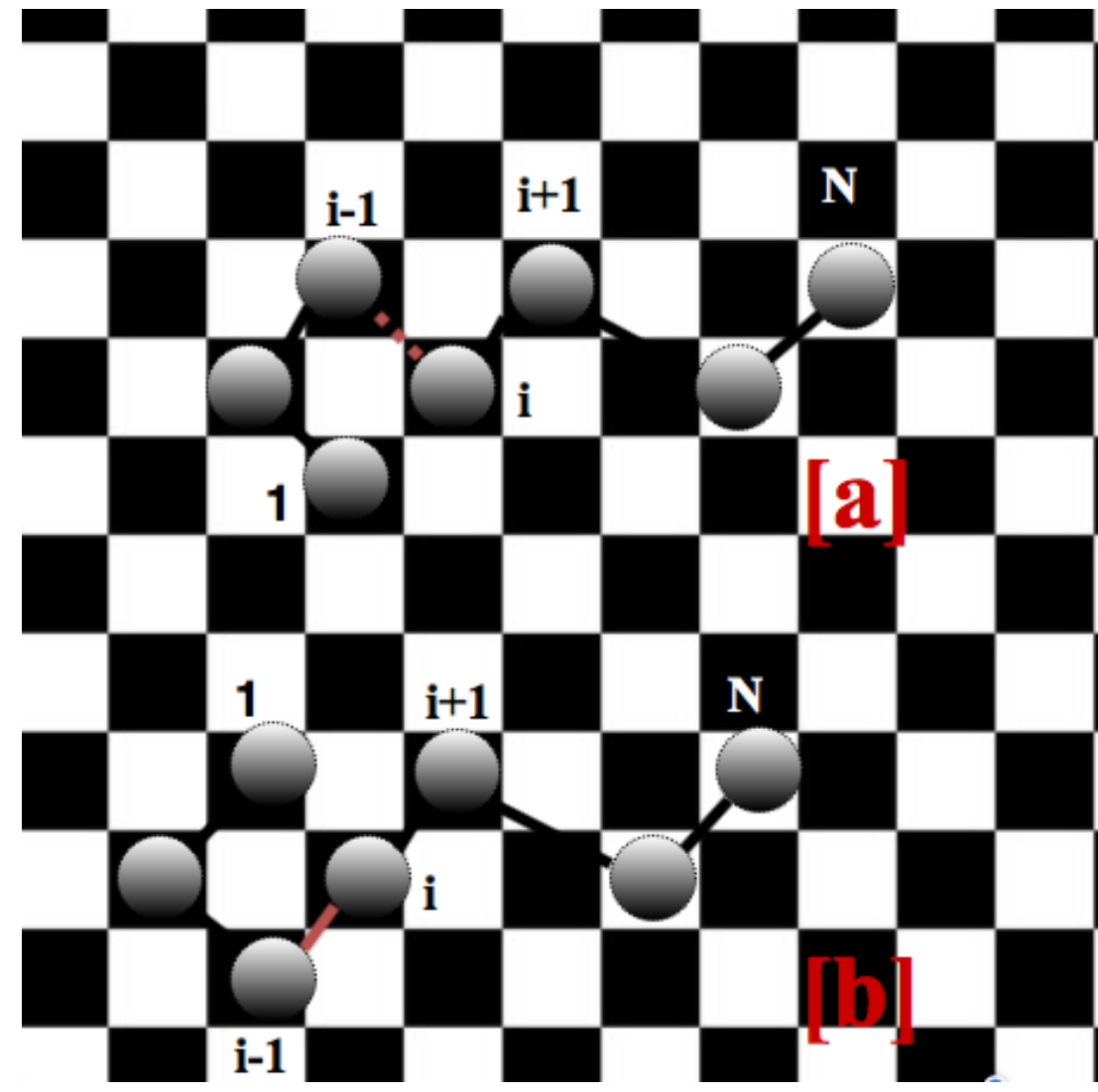

Figure 5.2: A state before conformation change is shown. $\mathrm{i}$ is the selected monomer and it is close to the end of the polymer chain, therefore in the second picture monomer makes a new bond with n-th monomer and looses its other bond with $\mathrm{i}+1$. Now $\mathrm{N}$ becomes i+1-th monomer and the previous neighbor will become the last monomer.

\subsubsection{Swap between sub-ensembles}

At this step a subsystem with a fixed configuration is checked for a transition from its current temperature to the next nearest neighbour temperature that is more stable from an energetic level point of view:

$$
\begin{aligned}
& w_{m m^{\prime}}\left(\beta_{m} \rightarrow \beta_{m m \pm 1}, q_{i}=\text { constant }\right) \\
& \quad=\min \left\{1+\exp \left[-U\left(q_{i}\right)\left(\beta_{m \pm 1}-\beta_{m}\right)+\eta_{m \pm 1}-\eta_{m}\right]\right\}
\end{aligned}
$$

where $\beta_{m}$ is the temperature of the current subensemble. We check the neighbours of this subensemble to see whether their energies are less than the current subensemble. $q_{i}$ is the conformation of polymer, it does not change during the temperature transformation.

In the whole MC process, there were $n_{m}$ MC steps in subensemble $\mathrm{m}$ at $\beta_{m}$ and $n_{m+1}^{\prime}$ MC steps in subensemble $m+1$ with $\beta_{m+1}$. If the total number of MC steps was 
$\mathrm{n}$ then the probability of occurence of subsensemble $\mathrm{m}$ is equal to $p_{m}=n_{m} / n$. As a result from (Eq. 5.10) $p_{m}$ will be $p_{m}=\frac{Z_{m} \exp \left(\eta_{m}\right)}{Z}$. Once we have found the ratio of two probabilities we can use the (Eq. 4.49) from chapter 4 for free energy difference of two subensemble $p_{m} / p_{m^{\prime}}$ :

$$
\beta_{m} F_{m}-\beta_{m^{\prime}} F_{m^{\prime}}=-\ln \left(p_{m} / p_{m^{\prime}}\right)+\eta_{m}-\eta_{m^{\prime}}
$$

The simulation is started using simple known partition function. For example, in our case we assume the initial system is at a very high temperature $(\beta=0)$ and consists of a gas of non-interacting monomers. By considering 6 degrees of freedom for each site, the partition function will become $\left(Z_{0}=6^{L}\right)$ and the weight factor $(\eta)$ will be zero. Therefore equation . 5.13 will transform into:

$$
\beta_{m} F_{m}=\beta_{0} k T \ln \left(Z_{0}\right)-\ln p_{m} / p_{0}+\eta_{m}=-\operatorname{Lln}(6) \ln \left(p_{m} / p_{0}\right)+\eta_{m}
$$

This equation defines the free energy $F_{m}$ is excess free energy from the ground state. In order to ensure balance of probability of occurrence for each subensemble, we need to find the optimal values for $\eta$, which requires a separate calculation. The initial values of $\eta$ also not known but we choose $\eta_{0}^{0}=0$ in the first run for the ground state. By combining $U(q)=\partial \beta F(\beta) / \partial \beta$ and from (Eq. 5.13) at equilibrium $\left(\ln \left(p_{m} / p_{m-1}\right)=0\right)$, thus:

$$
\eta_{m}^{0}-\eta_{m-1}^{0}=\left(\beta_{m}-\beta_{m-1}\right)\left(U_{m}(q)+U_{m-1}(q)\right) / 2
$$

We stop running the process once $p_{m} \simeq p_{m^{\prime}}$ and $\eta_{m}^{q}$ become the optimal value of $\eta$ after running $q$ times. After finding the optimal values of $\eta_{m}$ for each subensemble we can put the ultimate values in (Eq. 5.14) and follow the rest of the calculation in the equilibrium state.

We consider a range of temperature from $\beta=0, \ldots 4 \epsilon_{m m}^{-1}$ and for accuracy purpose, we divided this range of beta into 40 segments $(\Delta \beta=0.1), 20$ segments $\left(\Delta \beta=0.2 \epsilon_{m m}^{-1}\right)$, and 
10 segments $\left(\Delta \beta=0.4 \epsilon_{m m}^{-1}\right)$ and we compare the results to see how many subensembles are best. It turns out the number of subensembles should not be too small or too large, as when the number is too small, we may lose some important phases of the system and when the number of subensembles is too large, it is hard for the system to find the equilibrium state and it may become stuck in one ensemble, since the energy difference becomes quite high, therefore, the bias potential does not let the system to change its ensemble. The optimal number of subensemble will be different for different systems. For example, for an electrolyte system, people consider the range of $\beta=0 \epsilon^{-1}$ to $\beta=20 \epsilon^{-1}$ and then divide this range into 8 equal parts[127]. In our system, we found that most of the interesting phase behaviour occurs at temperatures from $\beta=0 \epsilon^{-1}$ to $\beta=4 \epsilon^{-1}$, since some phase behaviour occurs over a narrow range of temperature. We considered a small range of temperature in order not to miss any important evolution of the system.

\subsection{Results}

We start by finding the initial $\eta$ from a number of overlaps and adsorbed monomers and we obtain the optimal values of $\eta_{m}$ after three runs. Finding $\eta$ for a system that does not have any attractive sites is straightforward, but in the presence of attractive sites, the competition between overlap energy and adsorption energy makes the value of $\eta$ more complicated, since the balance point of forces will not change linearly over a range of temperatures and the equal probabilities of subensembles take a long time. Table. 5.1 shows the free energies for a polymer adsorbed over striped pattern of attractive site of size $L=2$. We did three runs for each pattern to find the optimal value and we normalized the value over the length of the box $D=24$ (see figure 2.7). The first column shows the values of $\beta$ in each subensembles and the second column is the total potential energy of short range and long range interaction. We assume the energy of binding monomer-surface $\left(\epsilon_{m s}=1 \beta^{-1}\right)$ and overlap energies of monomer-monomer $\left(\epsilon_{m m}=\epsilon_{a d}=1\right)$. At very high temperature $\left(\beta=0 \epsilon^{-1}\right)$ the fluctuation of the system is very high, so the high entropy of the system does not let the polymer chain to become close to the interface. Therefore at very high temperature the contribution of adsorption energy is nearly zero, while at low-temperature system has less entropy and tends to become stable as a consequence the there is only adsorption energy term in the total potential energy. The third column in this table shows the probability of occurrence of 
different subensembles becoming equal. It means that the balance factor is performing well.

\subsubsection{Probability of Desorption state of homopolymer chain onto het- erogeneous surface}

The temperature at which the polymer starts to become adsorbed on the surface is an important phase boundary for us. At this temperature, the energy of the polymer will be strong enough to make a new bond with the surface and overcome its internal interaction. Our system consists of two types of sites attractive sites and neutral sites: The polymer chain needs to distinguish the attractive sites and become adsorbed on those sites. We produced three different patterns of attractive sites in our system: random, striped and checkerboard. We also extend the size of each pattern to examine the effect of the size of islands on the temperature of adsorption of the chain. As a consequence, this phase can occur at different temperatures based on the conditions of the system. In order to understand when a system reaches this state, we can calculate the probability of desorption. The probability of desorption is computed from the total number of desorbed states at a particular temperature. At high temperature this probability is near to one; as the temperature goes down this probability will tend to zero.

\subsubsection{Adsorption energy of homopolymer chain onto heterogeneous surface}

Polymer adsorption has been studied extensively in chemically and biologically homogeneous surfaces. However, in reality, homogeneous surfaces do not exist. There are two types of heterogeneities on the surfaces: chemical and physical heterogeneity. Chemical heterogeneity results in some surface sites being preferable for adsorption than others, and physical heterogeneity results in some roughness and corresponding surface defects. We use a lattice model, so the size of each unit cell is comparable to the size of monomers in the solution. The surface is modeled as a flat square lattice with attractive and unattractive sites. In a periodic lattice the standard of measuring the adsorption energy of the system is the total number of adsorbed monomers on attractive sites times the binding energy; in this case $\epsilon_{m s}=\epsilon_{a d}=1 \beta^{-1}$, whereas in random lattice due to the different dimensionality of the attractive sites, we measure the adsorption energy 
TABLE 5.1: Striped pattern with size of islands $L=2$. The first column shows the $\beta$ segmentations. The second column computed the total potential energy of the system. The third column shows the probability of the subensemble occurrence in equilibrium. Three columns of $\beta_{m} F_{m}^{0} / L, \beta_{m} F_{m}^{1} / L$ and $\beta_{m} F_{m}^{2} / L$ show 3 times running to find the optimal value of free energy difference of subensembles.

\begin{tabular}{cccccc}
\hline$\beta_{m}$ & $U_{m}(q)$ & $p_{m}$ & $\beta_{m} F_{m}^{0} / L$ & $\beta_{m} F_{m}^{1} / L$ & $\beta_{m} F_{m}^{2} / L$ \\
\hline 0 & 4.8 & $4.45 * 10^{-2}$ & 0 & 0 & 0 \\
0.2 & 2.65 & $7.34 * 10^{-2}$ & 0.0298 & -0.457615197 & -0.663467 \\
0.4 & 1.95 & $4.46 * 10^{-2}$ & 0.0482 & -0.293948829 & -0.621584892 \\
0.6 & 1.1 & $5.12 * 10^{-2}$ & 0.0604 & -0.212847471 & -0.623527527 \\
0.8 & 0.75 & $5.10 * 10^{-2}$ & 0.0678 & -0.16571258 & -0.588376999 \\
1 & -0.35 & $4.94 * 10^{-2}$ & 0.0694 & $-9.28 \mathrm{E}-02$ & -0.344592154 \\
1.2 & -4.45 & $4.64 * 10^{-2}$ & 0.0502 & $3.73 \mathrm{E}-02$ & -0.207179055 \\
1.4 & -10 & $5.03 * 10^{-2}$ & -0.0076 & $-9.71 \mathrm{E}-03$ & $-2.06 \mathrm{E}-02$ \\
1.6 & -12.45 & $4.77 * 10^{-2}$ & -0.0974 & $-9.35 \mathrm{E}-02$ & -0.109515771 \\
1.8 & -14.8 & $4.68 * 10^{-2}$ & -0.2064 & -0.20102872 & -0.202600151 \\
2 & -15.4 & $4.50 * 10^{-2}$ & -0.3272 & -0.303032279 & -0.308999509 \\
2.2 & -19.45 & $4.46 * 10^{-2}$ & -0.4666 & -0.464121163 & -0.574201226 \\
2.4 & -20.2 & $4.50 * 10^{-2}$ & -0.6252 & -0.626578093 & -0.621058524 \\
2.6 & -19.6 & $4.46 * 10^{-2}$ & -0.7844 & 0.78000176 & -0.780239105 \\
2.8 & -18.8 & $4.58 * 10^{-2}$ & -0.938 & 0.929854214 & -0.931794882 \\
3 & -19.8 & $4.46 * 10^{-2}$ & -1.0924 & -1.12098658 & -1.09032285 \\
3.2 & -18.4 & $4.55 * 10^{-2}$ & -1.2452 & -1.25291169 & -1.25037634 \\
3.4 & -19.35 & $4.54 * 10^{-2}$ & -1.3962 & -1.39173567 & -1.39034104 \\
3.6 & -19.35 & $4.51 * 10^{-2}$ & -1.551 & -1.50077653 & -1.50024617 \\
3.8 & -20 & $4.47 * 10^{-2}$ & -1.7084 & -1.78565001 & -1.78224671 \\
4 & -22 & $4.46 * 10^{-2}$ & -1.8644 & -1.86106634 & -1.86087024 \\
\hline & & & & &
\end{tabular}

based on the coverage of the surface by adsorbed monomers $\left(N_{m}\right)$ over the total number of attractive sites $\phi=N_{m} / N$. Our goal here is to study the effect of distribution and size of heterogeneous sites on adsorption states. Moreover, this information will help determine whether the pattern and length scale of that pattern are important factors in the desorption transition of the system[174].

\subsubsection{An overview of four different phases of the system}

There are four important phases that we want to study in this system: fully crumpled, expansion, adsorption, and pattern recognition. Before studying different patterns and comparing them together, we show these four phases by an example. From snapshots you can see these four phases step by step (Figure 5.4 and 5.5). First the polymer is fully crumpled then it starts to expand and become adsorbed on the surface and in the 


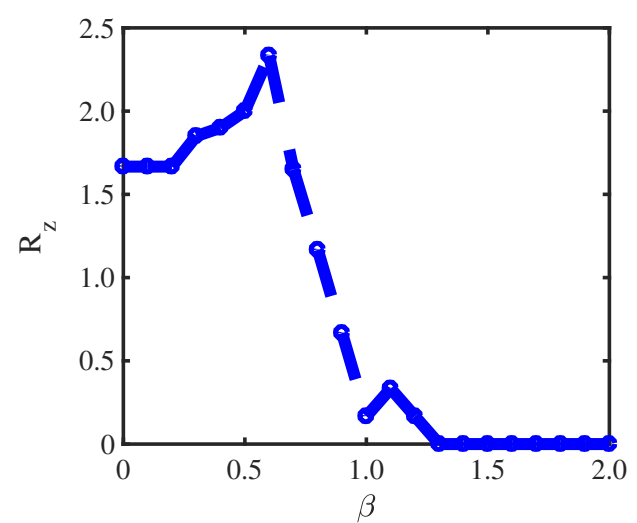

(A) Centre of mass in the $\mathrm{z}$ direction for $\mathrm{H}$

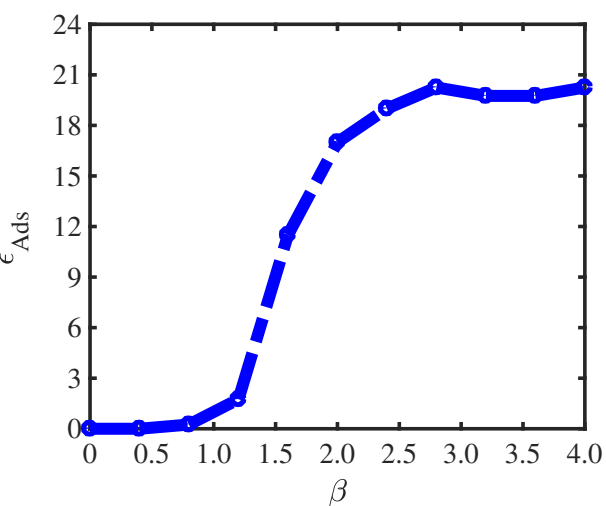

(C) Adsorption enery for striped pattern with active site $L=2$

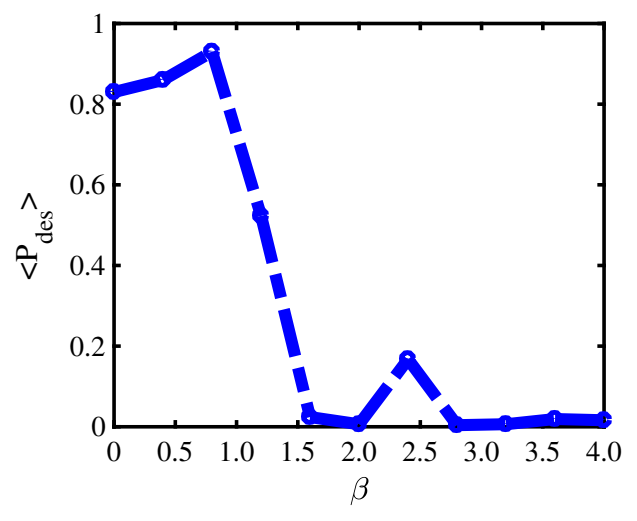

(B) probability of desorption state for striped pattern with active site $\mathrm{L}=2$

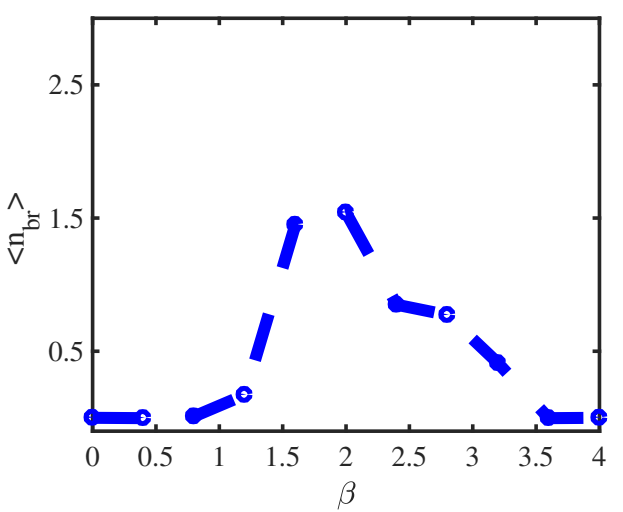

(D) Average number of bridges of striped pattern with active sites $L=2$

Figure 5.3: This figure shows a summary of four important phases that occur during simulation. All the figurs are in striped pattern with $L=2$. In figure (A), line drop from maximum to minimum shows the transition from fully crumpled to the expanded state. Figure (B) demonstrates the probability of the number of desorbed state in the system. Figure (C) shows the transition from desorbed state to adsorption state. Saturation in high $\beta$ shows fully adsorption of the chain. Figure (D) show the pattern recognition by polymer chain. Once the total number of bridges tend to zero, the polymer has fully recognized the pattern of the surface.

last step the chain tries to recognize the surface. After simulating different phases, we want to draw the phase diagram of the system that the length of the attractive islands and the temperatures are two key factors of its phase transitions. Figure. 5.3 shows the different phases of the striped pattern at $\mathrm{L}=2$.

In order to understand these phases better, we took some snapshots of different phases for different patterns. We consider only one length of island size for three different patterns to understand the evolution of the system as $\beta$ increases. 


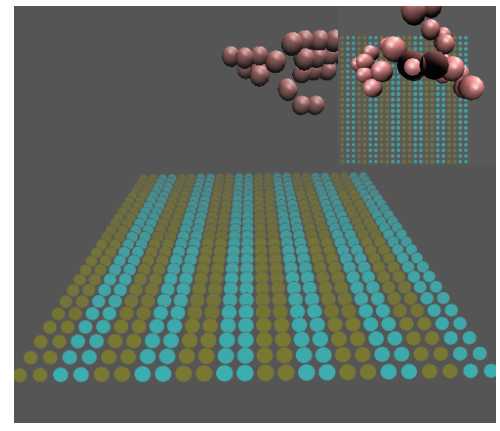

(a)

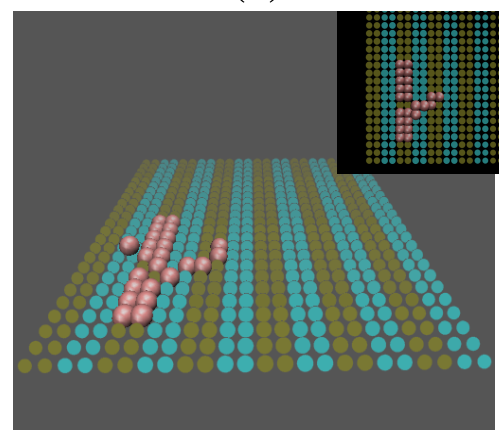

(c)

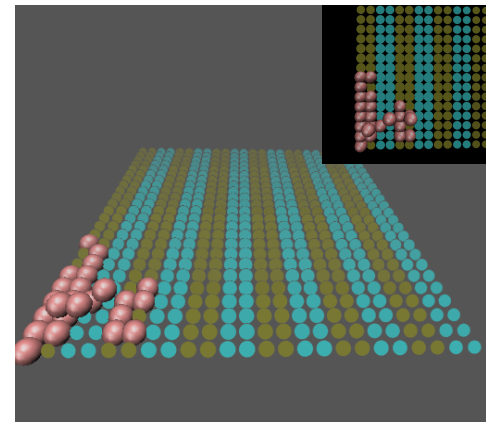

(b)

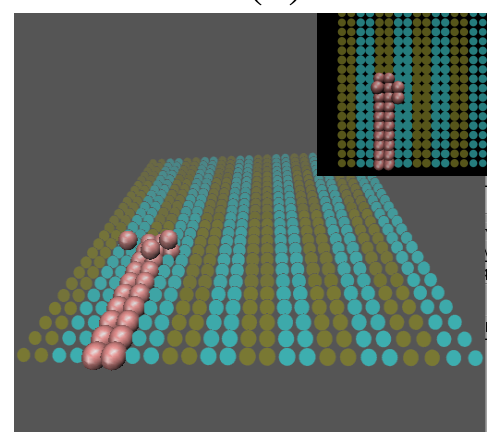

(d)

(A) Different snapshots of striped pattern at different range of temperatures: very high $=(\mathrm{a})$ to low temperature $=(\mathrm{d})$. From a to $\mathrm{d} \beta=0.0 \epsilon^{-1}, 1.2 \epsilon^{-1}, 2.5 \epsilon^{-1}, 3.5 \epsilon^{-1}$

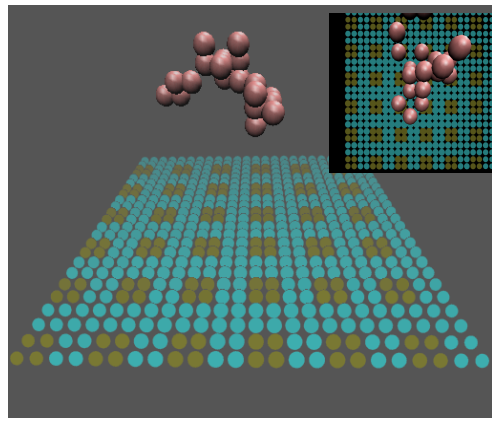

(a)

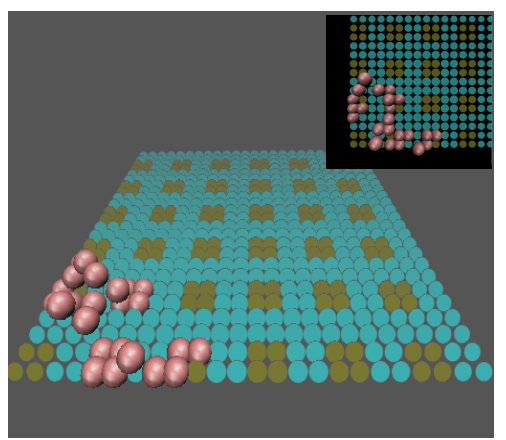

(c)

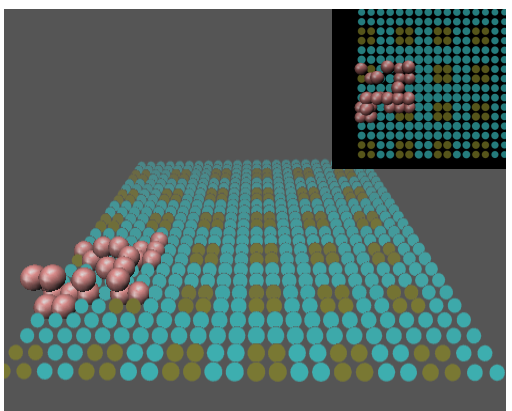

(b)

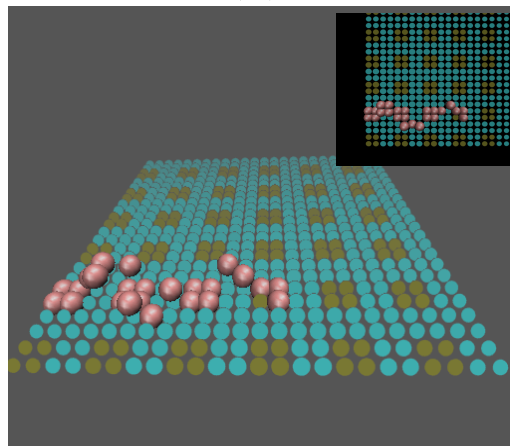

(d)

(B) Different snapshots of checkerboard at different range of temperatures: very high $=(\mathrm{a})$ to low temperature $=(\mathrm{d})$ From a to $\mathrm{d} \beta=0.0 \epsilon^{-1}, 2.0 \epsilon^{-1}, 3.0 \epsilon^{-1}, 4.0 \epsilon^{-1}$

Figure 5.4: The snapshots of two different patterns at four different temperatures with $\mathrm{L}=2$. The brown sites show the attractive islands and green sites or neutral. 


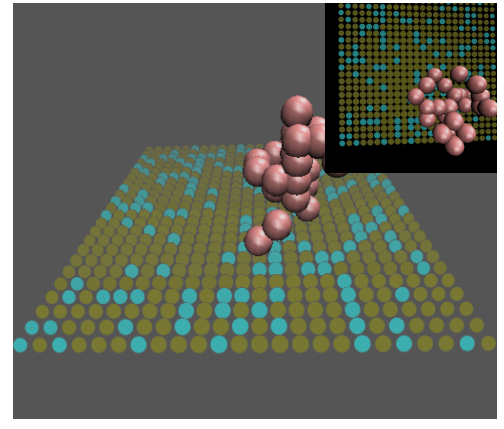

(a)

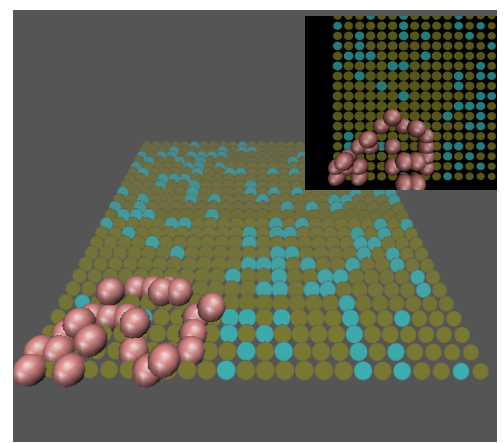

(c)

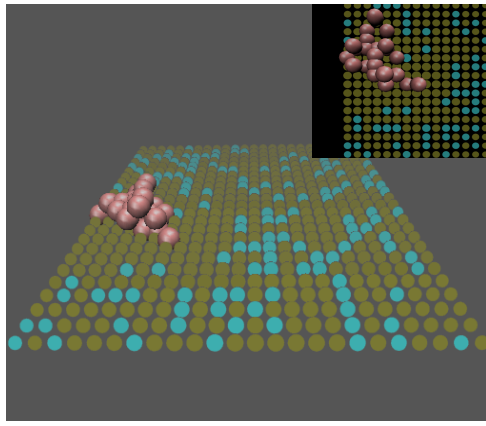

(b)

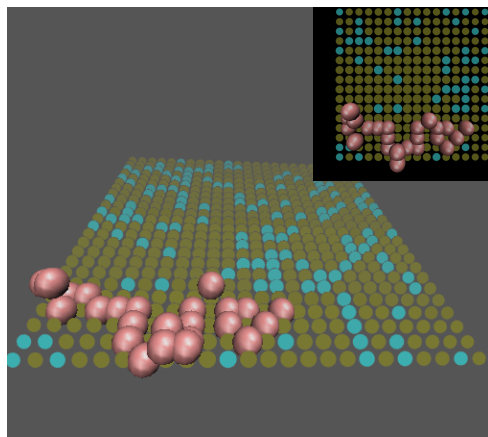

(d)

FiguRE 5.5: Different snapshots of one arbitrary random pattern (does not change over time) at different range of temperatures: very high $=(\mathrm{a})$ to low temperature $=(\mathrm{d})$ From a to $\mathrm{d} \beta=0.0 \epsilon^{-1}, 1.8 \epsilon^{-1}, 2.5 \epsilon^{-1}, 4.0 \epsilon^{-1}$.

\subsubsection{Expansion of homopolymer adsorption}

We begin with an analysis of the effect of the distribution of the active sites on the geometry of the homopolymer during adsorption. One of the most important quantities is the end-to-end vector of polymer in the z-direction, which helps us first to find the radius of gyration and moreover, gives us some insights about the elasticity of the chain for different ranges of temperature. This measurement directly helps us to find the transition from fully crumpled polymer chain to expanded polymer chain that is ready to become adsorbed on the surface. (Figure. 5.10) the temperature and pattern scale dependencies of $\left\langle R_{z}\right\rangle$. For a checked pattern with $L=1$ or $L=2$, the degrees of freedom for adsorption of the segments is less than for a striped pattern, and therefore, the entropy of the system in the checked pattern with size $L=1$ or $L=2$, is higher than the other two patterns[175]. As we can see, the length of the polymer chain in the $\mathrm{z}$ direction in this pattern is higher than that of the striped and random[176]. However, as we increased the size of the domain sites in the checked pattern, the number of degrees of freedom or available sites in this pattern become greater and comparable with the 


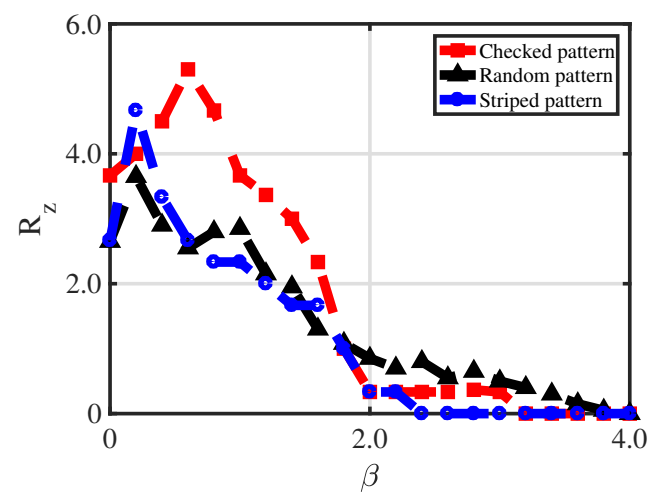

(A) Centre of mass in the $\mathrm{z}$ direction for active $\mathrm{H}$

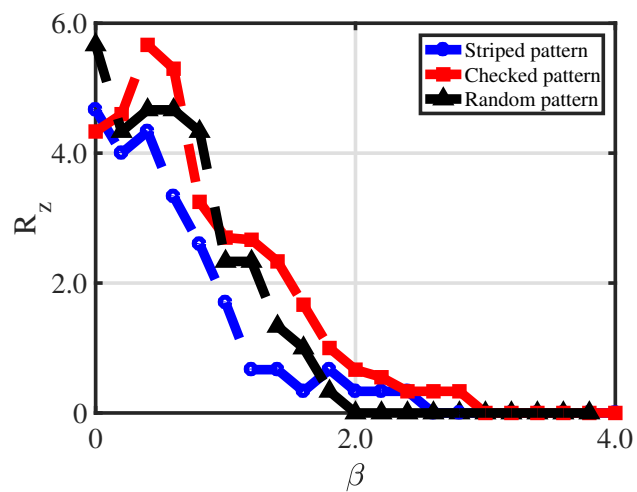

(C) Centre of mass in the $\mathrm{z}$ direction for active site $\mathrm{L}=4$

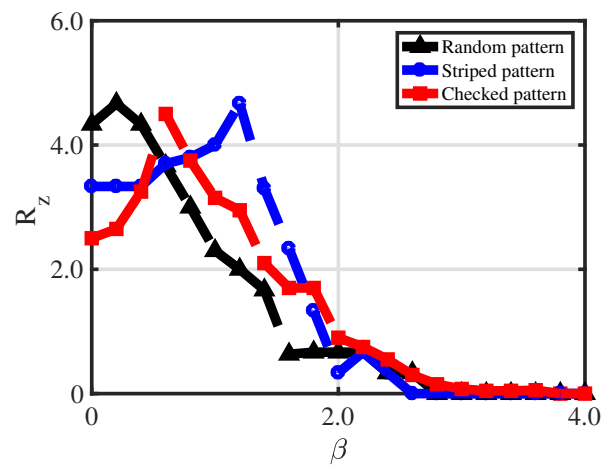

(B) Centre of mass in the $\mathrm{z}$ direction for active site $\mathrm{L}=2$

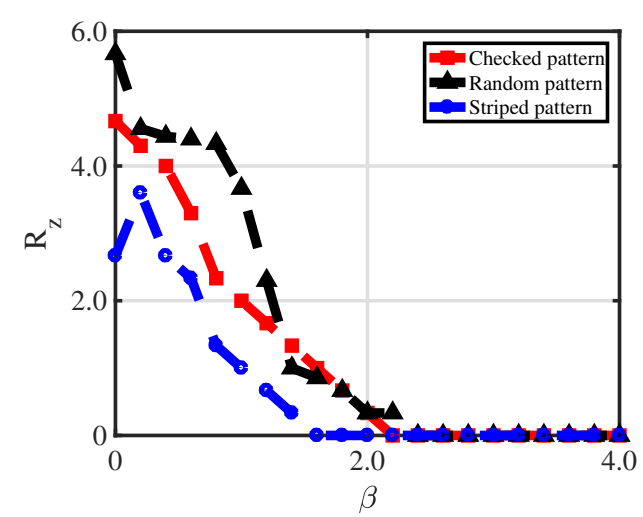

(D) Centre of mass in the $\mathrm{z}$ direction for active site $\mathrm{L}=16$

Figure 5.6: The average of centre of mass for different pattern and different size of active sites

striped. Due to the complexity of the random pattern, this pattern has the maximum length of an end-to-end vector in the z-direction.

The reason that the height of chain at $\beta=0 \epsilon^{-1}$ is not maximum in figure 5.6 is because of the number of overlaps. Basically, at $\beta=0$ (very high temperatures) the polymer is crumpled, and therefore the distance between the two ends is very short. Then at higher beta for example $\beta \rightarrow 1$ the chain becomes more expanded and once it comes to the surface, its height will tend to zero.

\subsubsection{Critical temperature of adsorption and desorption}

At $Z=0$ system has two types of sites: neutral and attractive sites. A few attractive sites can attach together and make an island. The total number of attractive sites 
divided by unit area defines the coverage $(\phi)$. The Adsorption energy is defined as a total number of the adsorbed monomer on the attractive sites times the unit of energy, which is $\epsilon^{-1}=k_{B} T$ :

$$
\epsilon_{\text {ads }}=\text { Nadsorbedmonomers } *\left(k_{B} T\right)
$$

The thermodynamic properties of the system at very high temperature and very low temperature are quite different. At very high temperature $\left(\beta \simeq 0 \epsilon^{-1}\right)$ the behavior of the polymer chain is similar to the ideal chain that we discussed in Chapter 4, and hence this is where we see the most compact form of the polymer chain. As the temperature decreases the polymer chain expands due to the excluded volume effect. The system transitions from the configuration of an ideal polymer chain to a real polymer chain with excluded volume. At this temperature, the polymer chain starts to become expanded and ready to be fully adsorbed on the surface since active sites can only accept one monomer per unit area, for adsorbing on the surface chain needs to become fully expanded first. Sometimes due to the high energy of the surface, the chain does not have a chance to expand itself, and therefore some monomers can never hit the surface. (Figure. 5.7) shows the total number of adsorbed monomers with different length over a different range of temperatures. At very high temperature no segment is adsorbed yet as $\beta$ increases a few monomers start to become adsorbed on the surface. This point is designated to be the critical temperature of adsorption. The number of adsorbed monomers is higher for wider domain size. While in strong adsorption states the number of adsorbed monomers will decrease with broader domain size. This is because of the time evolution of the system. In the presence of very strong adsorption sites the system does not have any chance to self-assemble itself onto the attractive sites. Therefore, many monomers that have overlap together do not have enough time to find the private place for themselves (see Figure. 5.7). At small domain size, this critical point for a pattern like stripe occurs at a higher temperature than complex patterns like checked and random. Another important feature that we can see in the figures is the total number of adsorbed monomer at saturation points, which for pattern like checked with domain size $L=1$ this value cannot exceed further than $N_{a d s}=8$, whereas this value for more simple pattern like striped at the same size is almost twice.

The critical temperature of desorption refers to the fraction of a total number of 


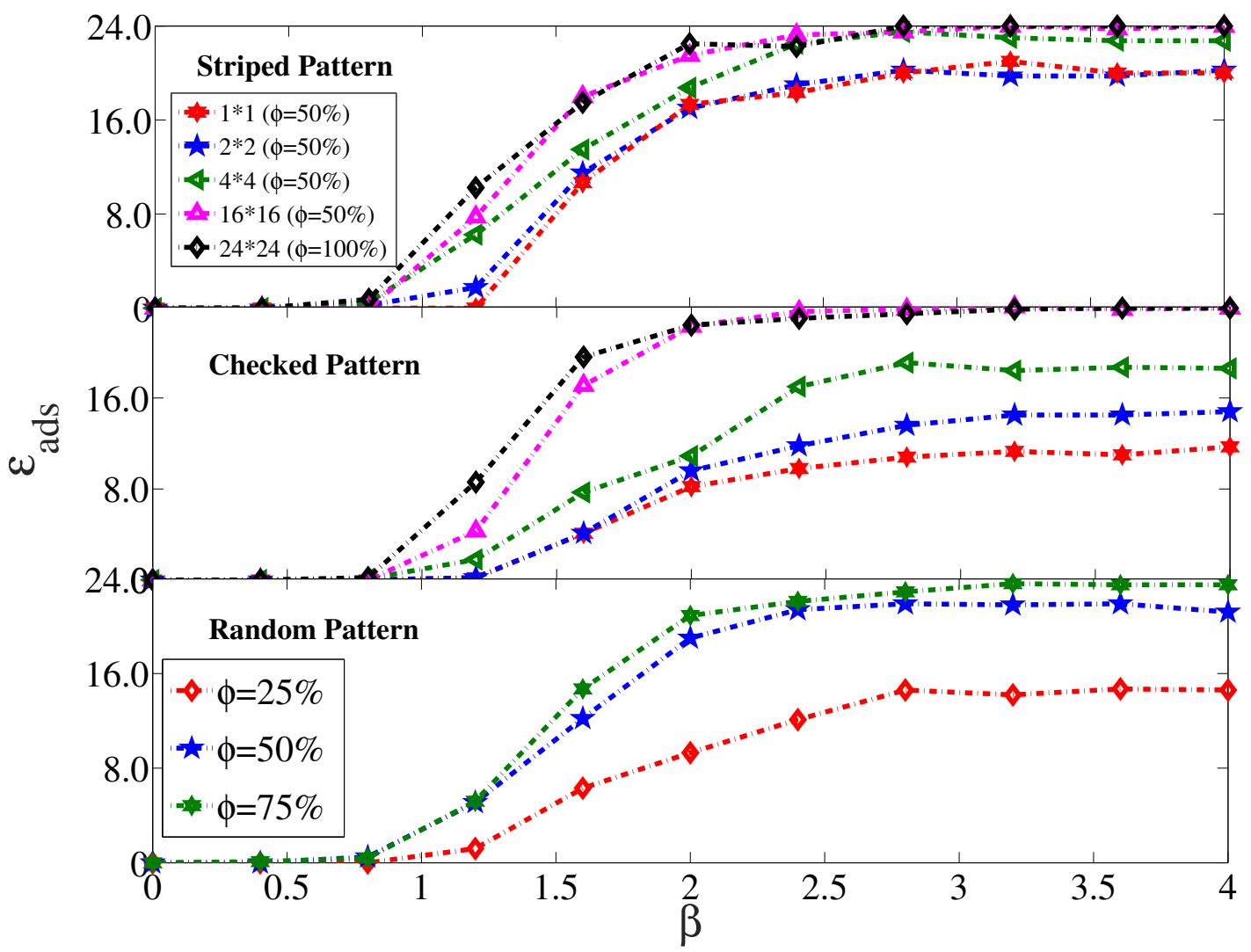

Figure 5.7: The energy of adsorption for different patterns with a different size of the sticky island. From this figure, we can find the critical temperature of adsorption for different types of adsorption sites. Once a few monomers start to become adsorbed on the surface we measure this temperature as a critical temperature of adsorption. The $\phi$ values of striped and checkerboard pattern are the same.

states that are in desorbed state and this number for high temperature is equal to one and it means that $100 \%$ of the states are in desorbed states. As the temperature goes down the probability that polymer found in desorbed states is decreasing. This fraction suddenly drops off once a few monomers adsorb on the surface. This is the point we measured as the critical temperature of desorption (see Figure 5.8).

\subsubsection{Pattern Recognition}

The size of domain sites helps us to understand the interplay between the size of active sites and monomer pattern recognition. Moreover, the strength of adsorption is another factor that can alter the temperature of recognition[177]. To distinguish between highly adsorbed and recognizing surface (see Figure. 5.9a). This may not be very important for a single chain, while it plays a key role in the crowded polymer chain; when applying the polymer chain it is very expensive and we want to take advantage of 


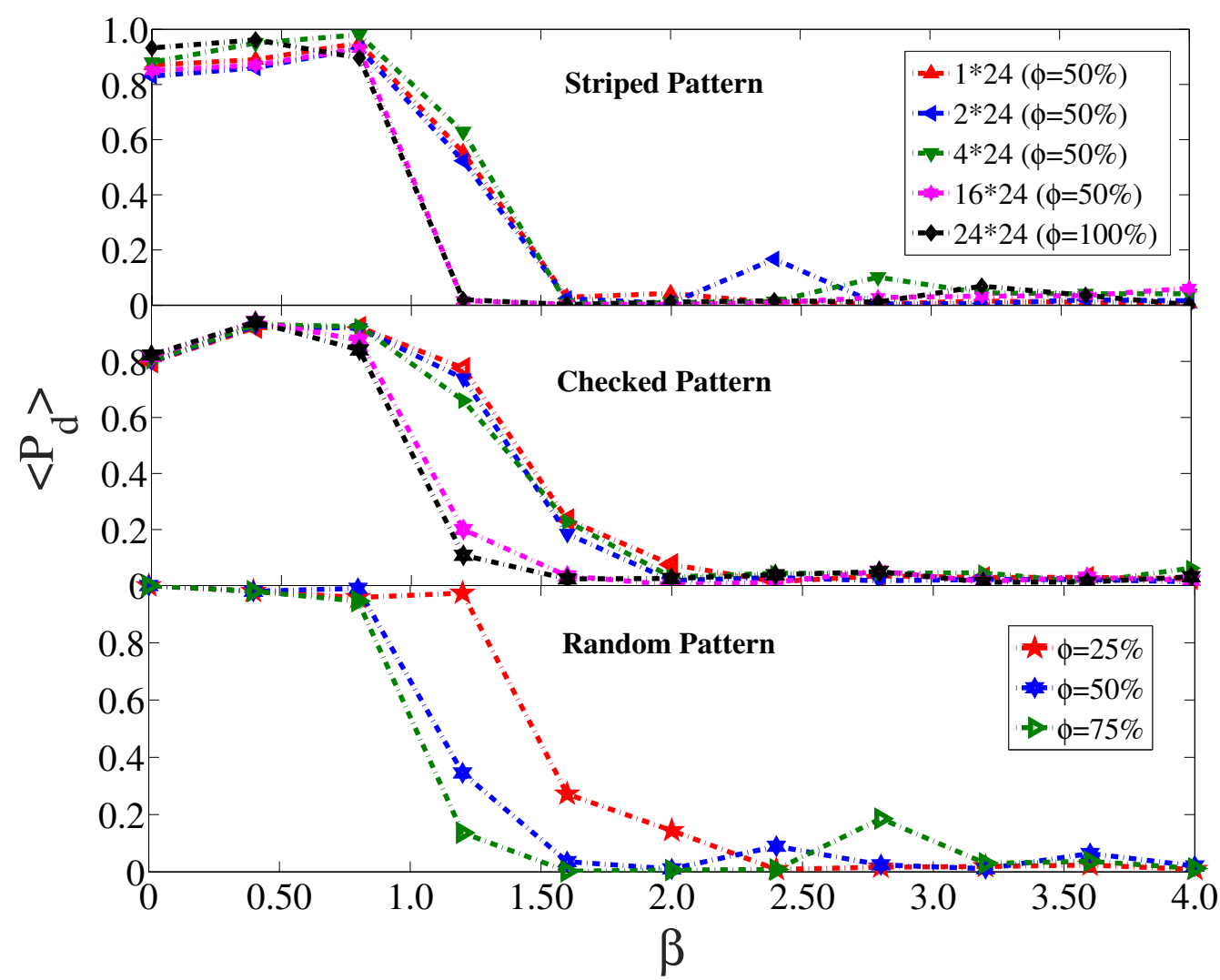

FiguRE 5.8: This figure shows the probability of desorption for different patterns with a different size of the attractive sites. From this figure, we can find the critical temperature of desorption for different types of attractive sites. Once a few monomers start to drop on the surface the total number of desorbed states start to become decreased and we can measure the temperature as a critical temperature of desorption. $\phi$ values of checkerboard and striped pattern are the same.

the maximum efficiency of this material[178]. Therefore the second sharp transition of the system is when the polymer chain recognizes the pattern and become fully frozen that pattern. Pattern recognition is very important in curing cancers by a smart drug delivery system through polymer chains[179]. In order not to waste this expensive material, they apply polymer chains not only to recognize the tumor cells but also to help drugs to optimize their life expansion on these patches. (Figure. 5.9a) shows the general concept of recognition. As you can see in the first figure (a) polymer chain is fully adsorbed and the energy of binding will be equal to the other figure (b). However, in the first figure there are some bridges and since this is not a stable phase for the polymer chain, it will tend to get off the surface. However, in figure (b) the polymer is more stable and fully relaxed at the pattern. (Figure. 5.9b) shows the average number of these bridges for two different patterns and two different sizes of islands. In both patterns at high temperature, there is no binding between the polymer and the surface, while at 


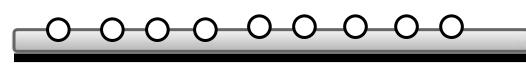

(b)
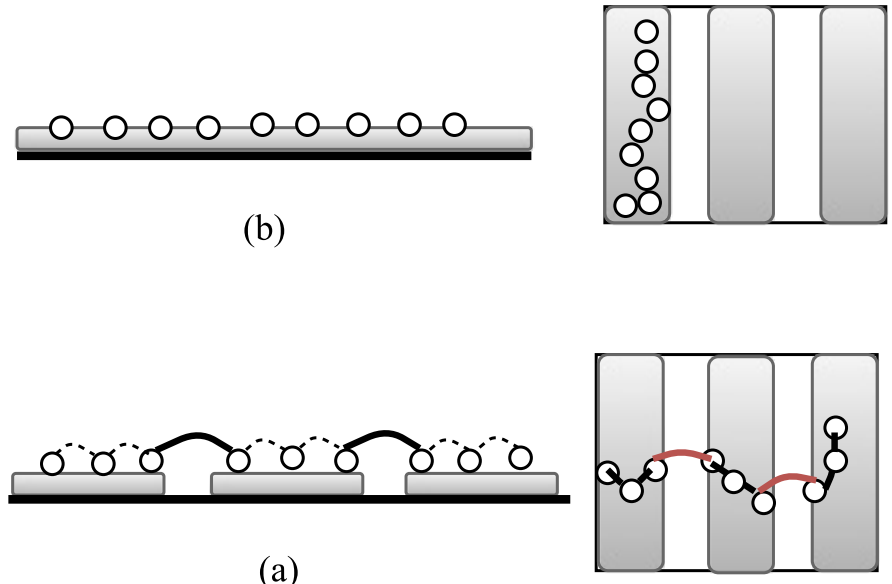

(A)

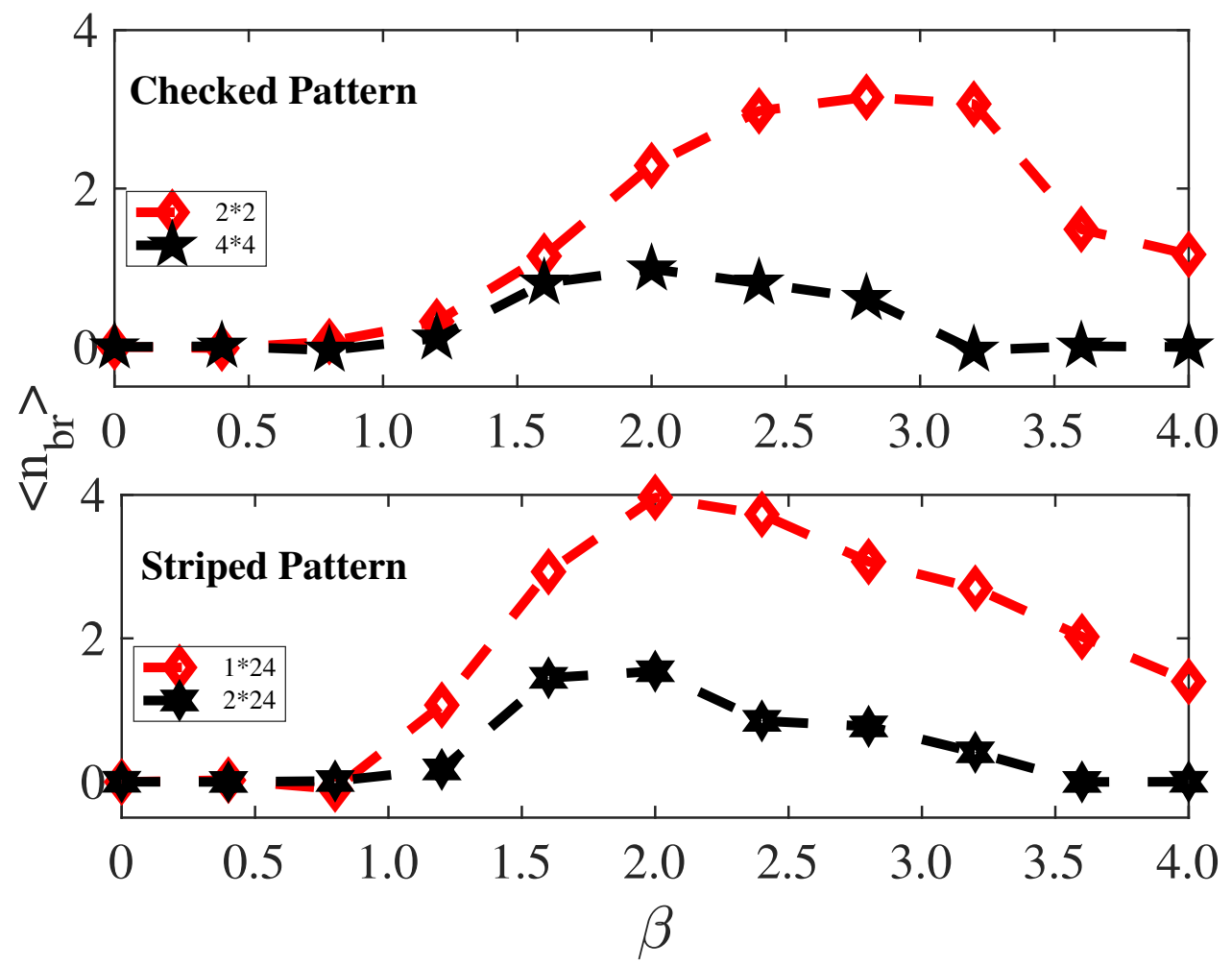

(B)

Figure 5.9: (A) Difference between fully adsorbed and the pattern recognition A-a polymer chain is fully adsorbed on the surface. Nevertheless, the chain makes several bridges from one attractive site to another. In A-b the surface is fully recognized by polymer chain (no monomer remains unabsorbed). For $\mathrm{L}=1$ polymer will never become adsorbed on one line unless the length of the chain was smaller than the size of island. (B) Numerical results of an average number of bridges in checked pattern and striped pattern for two sizes of attractive islands $L=2,4$. 
higher $\beta$ the chain becomes partially adsorbed. The number of bridges starts to increase by decreasing the temperature; for example, around $\beta=0 \epsilon^{-1}$ for the striped pattern the number of bridges is maximum, and then starts decreasing due to recognizing the pattern and once it is zero, the polymer has recognized the pattern.

\subsubsection{Phase diagram}

In the phase diagram of the system, four major phases can be discovered. 1) Desorption: at very small $\beta$ and very small $L$, at this phase system, has the highest and lowest binding energy. The polymer chain is fully crumpled and has the highest entropy. 2) Expansion: another phase is when $\beta$ becomes larger and $L$ is very small, polymer chain cannot adsorb but the overlap energy will decrease and the chain becomes expanded. The entropy of the system starts to decrease as the system becomes ready not to have overlaps. 3) Adsorption: at very low temperature and low to medium size of islands a few monomers start to adsorb on the surface, however not all the monomers can adsorb in this phase. 3) Pattern recognition: as we increased the length of the active sites, adsorption will become the main phase of the system. Polymer tends to adsorb on the surface faster and the number of adsorbed monomer increase until at a certain temperature and size of islands, polymer chain become fully frozen on the surface. As the size of the island tends to a maximum, which is the length of the box, almost all of the phases of the system will meet together at a certain point, which is the critical temperature of adsorption for homogeneous surfaces. Drawing a phase diagram for an ordered pattern such as checked or striped is easier than a disordered pattern like random, and therefore here we only stick to the accurate phase diagram of striped and checked patterns because we could calculate the exact numeric values for each phase of the system. For a random pattern understanding the critical temperature of adsorption and desorption is not very complicated; interpreting the pattern recognition temperature, however, is not very accurate.

The phase diagram of figures $5.10 \mathrm{a}$ and $5.10 \mathrm{~b}$ demonstrate for any long flexible ideal chain that the binding energy of the surface can overcome to the overlap energy of the monomers, the temperature of the phase transition is proportional to the length of domain size. 


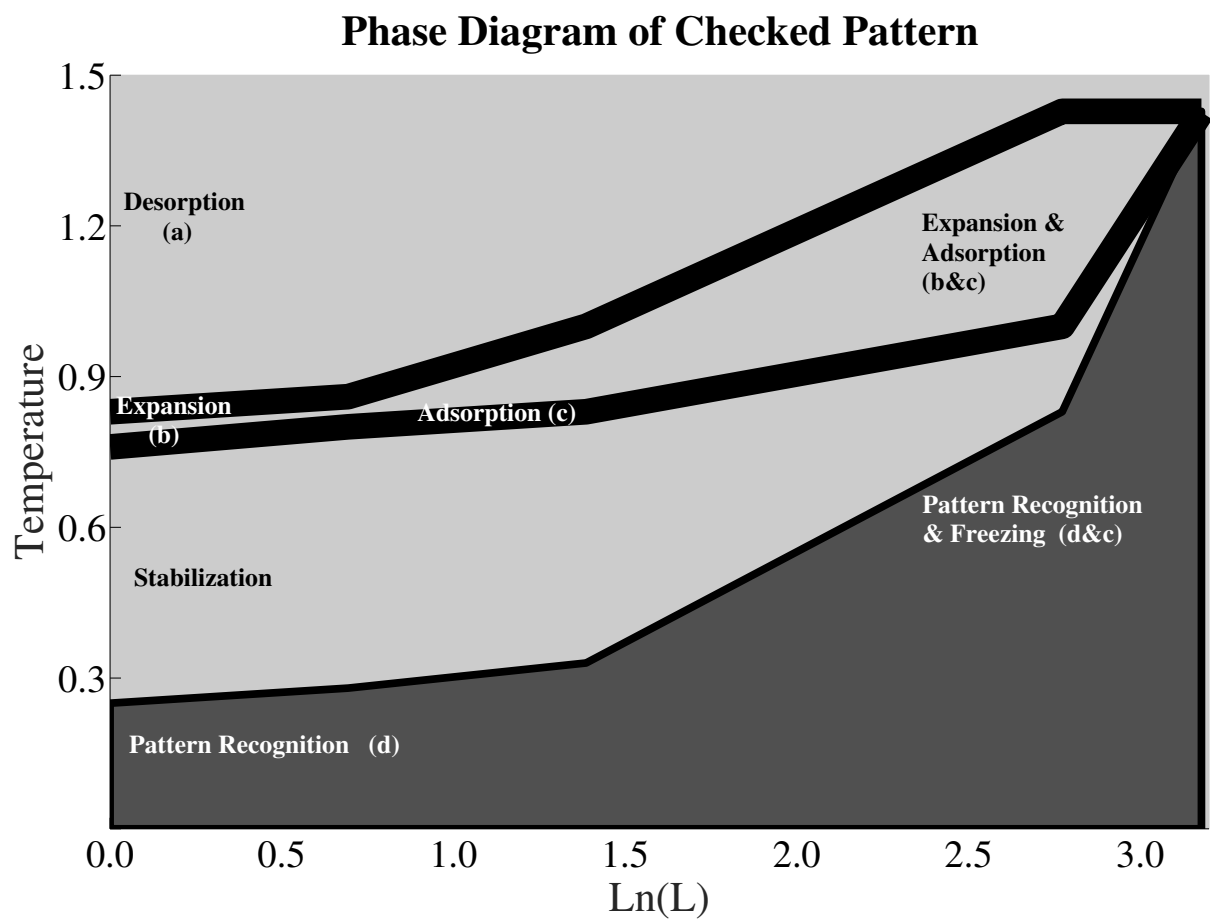

(A) Phase diagram of checkerboard by two order parameters: temperature and size of island

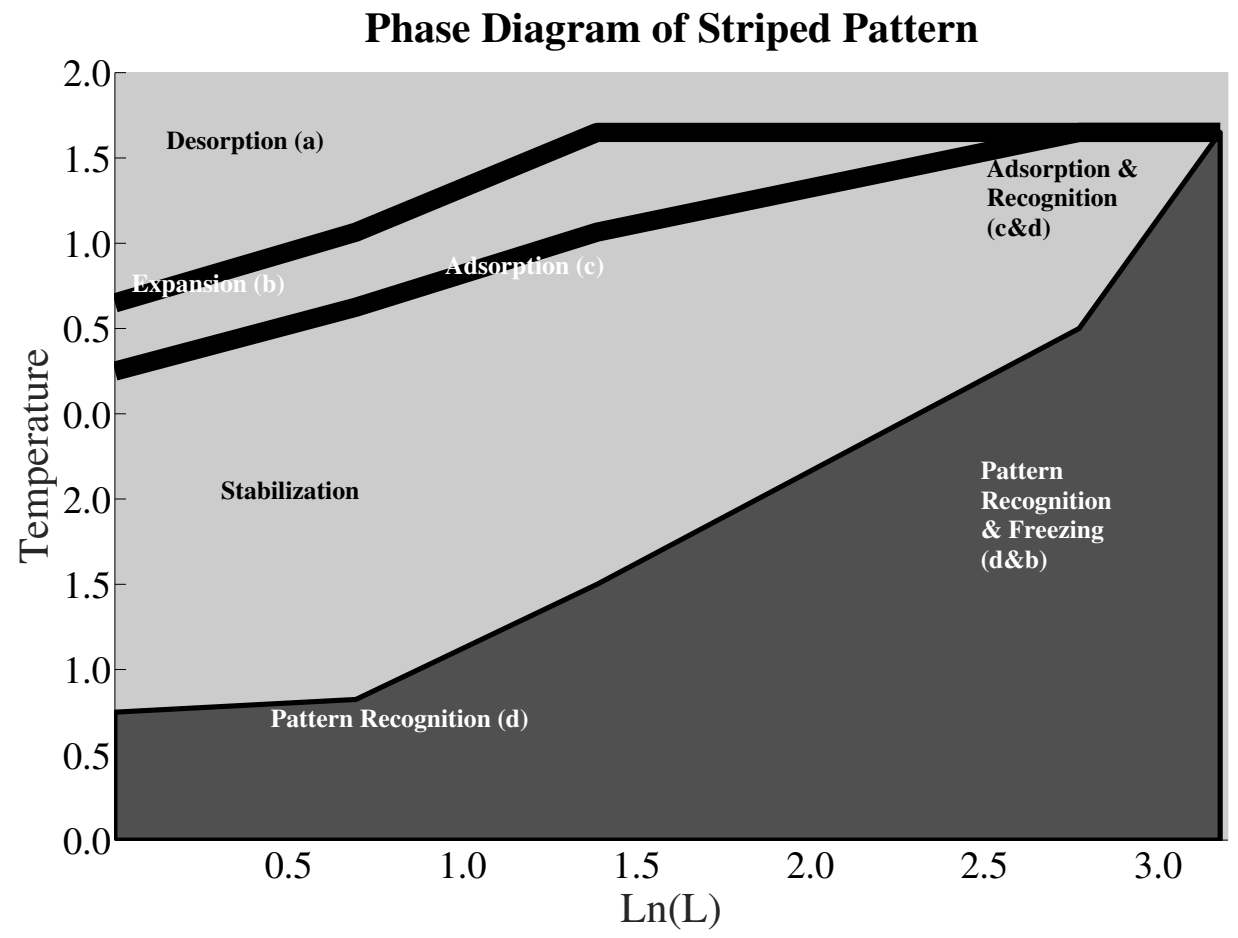

(в) Phase diagram of striped by two order parameters: temperature and size of the island

Figure 5.10: These two figures demonstrate the different phases of the system. At high temperature, the polymer is fully desorbed crumpled and compact (a) then starts to expand (b) and ready to make a new bond with the surface. After a few monomers have adsorbed on surface adsorption will start (c). In the next step polymer starts to find the stable state and become relaxed on the surface (d). The width of the lines show that the boarder between two regions is not very sharp and it changes from case to case. Therefore, we cannot separate two phases with a certain point. 


\subsubsection{Compatible Surfaces}

In this section, we consider the mixture of different surfaces only because in reality, different types of the facet can exist in the crystal structure, or in biology we have so many biocompatible surfaces, and as a consequence, we need to understand in this situation what pattern is the most desirable for a chain to adsorb on. From a statistical point of view, the polymer tends to adsorb on a place that has greater numbers of degrees of freedom, and therefore in the battle between checked and striped pattern, the striped pattern will win and attract the polymer chain on its sites.

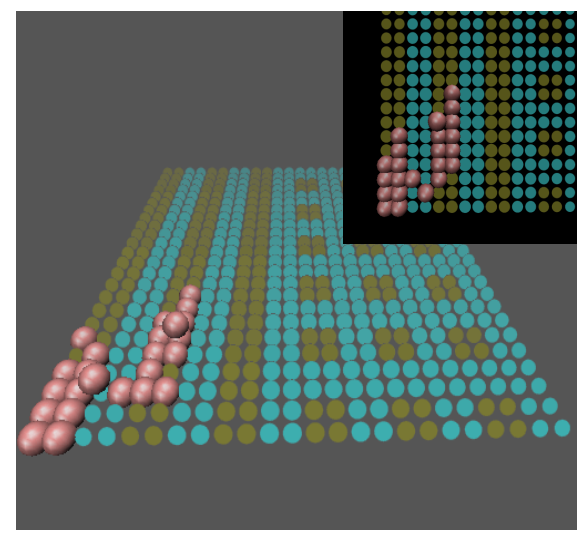

(a)

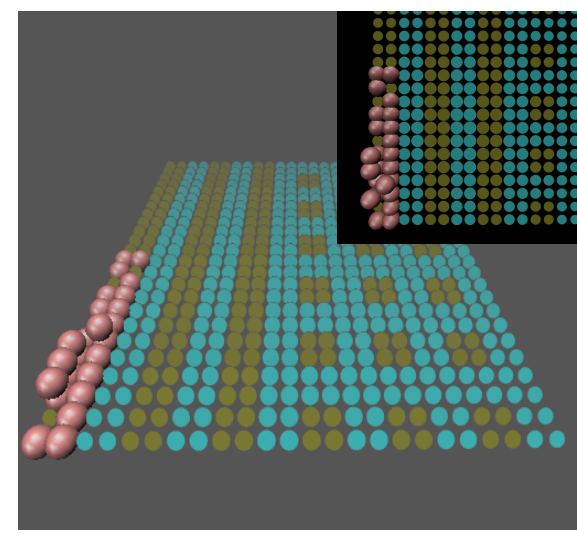

(b)

Figure 5.11: Compatible schematic to show the competition between striped and checked pattern for adsorbing polymer chain.

In order to test our observation, we ran our model 144 times to check the frequency of the adsorption of the polymer chain in different positions on the surface. Figure 5.12shows the histogram of this testing for each length $\mathrm{x}$ noted as the size of islands $L$. From $L=1$ to $L=12$ we designed a striped surface and beyond that is the checkerboard surface. Our testing demonstrates that the striped pattern is more desirable than the checkerboard for the polymer to be adsorbed on that. As you can see the frequency of adsorption in the striped pattern area is greater than the checkerboard. 


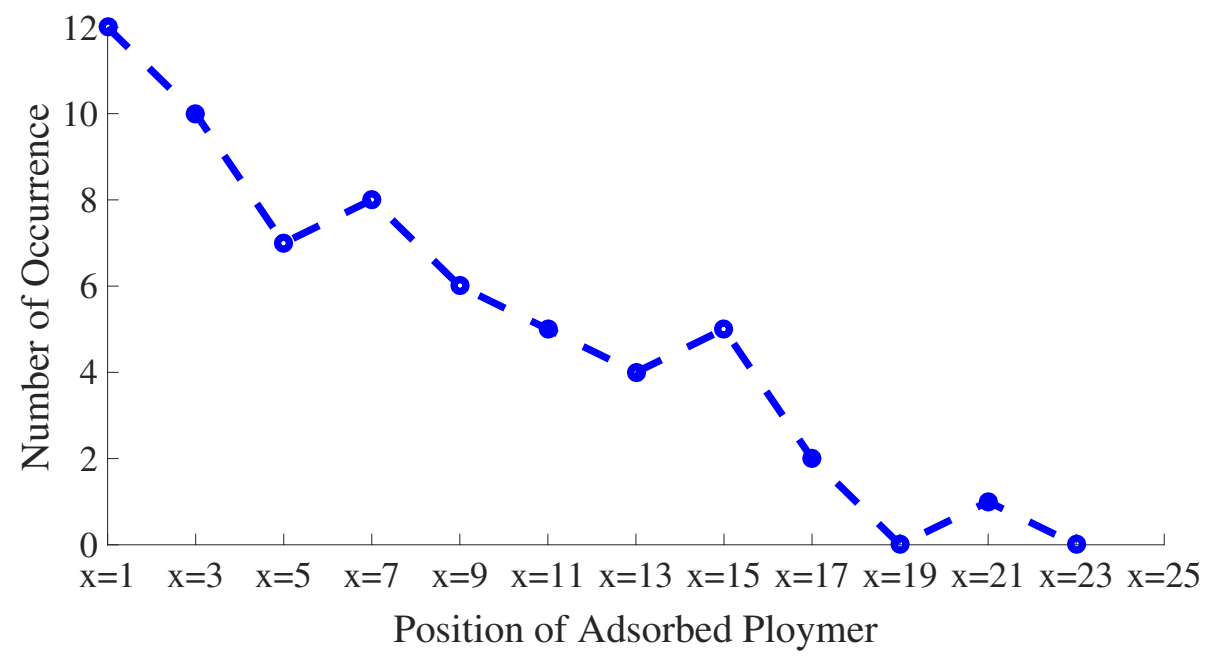

Figure 5.12: The histogram of adsorption of Polymer chain on a mixed surface demonstrates that stripe pattern is more desirable than checkerboard for polymer to become adsorbed.

\subsection{Conclusion}

In order to summarize all the things that we have found in this chapter, suppose a freely jointed chain (FJC) and heterogeneous attractive sites are dynamically in an interaction condition. Generally, we were interested in analyzing thermodynamical and statistical properties of the adsorbed state, in order to identify different types of surfaces. In addition, due to the importance of the pattern recognition in many biocompatible and chemical surfaces, we want to find the relation between phases and recognize the pattern of the surfaces. This will help us to understand what state the system will have maximum coverage of attractive sites by segments of the polymer chain. As a part of this study, we focus on different patterns of the surface, which can be a demonstration of different types facets on a crystal structure. As a result, the primary question was whether different patterns or structure of the lattice can affect on adsorption states and phase transition of the system. Additionally, when the system does stop evolving or in another words the polymer chain will be frozen on a specific structure.

The model that we chose was suitable for the conformation of the polymer, the flexibility of the coil and the competition between binding energy of the surfaces and fluctuation of configurations. The extended ensemble method due to the flexibility of transition within a different range of temperatures and measuring the entropy of such a stochastic problem at each temperature allows us to extract the important details of the thermodynamic behavior of the system from very high to low temperatures. 
Our result proved that in nanostructured formats of pattern FJC has more degrees of freedom in the striped pattern. Therefore, it has this capability to reach to the steady state at higher temperatures, a checked pattern is the most complex surface due to the limitation of accepting monomers on its attractive sites. This difference between patterns will fade away at lower temperature and broader domain sizes[180].

In the next chapter, we consider two types of monomers in a chain. Building and designing the best polymer chain for optimal results from the coating is a big challenge. One of these applications is in crystal growth. We will study this in the following chapter. 



\section{Chapter 6}

\section{Copolymer Adsorption onto Heterogeneous Surfaces}

The use of copolymers to influence non-uniform crystal growth across different facets has attracted a lot of attention recently[172]. Designing particular copolymers that modify the rate of adsorption near particular surfaces is a promising approach to optimising crystal structure. Different types of blocks can be assembled in a chain, including hydrophobic and hydrophilic blocks or double hydrophilic blocks, (A-block-B or A-alt-B). Here we assume our copolymer type is A-alt-B, which are usually small monomers with small chain lengths. If we need to alter the surface energy of a particular facet, specific copolymers can be designed to preferentially absorb on that facet. As a result, such copolymers are sometimes called crystal modifiers[181].

Here we study the selectivity of a copolymer on a heterogeneous surface with different strength of adsorption. The approach is similar to that for homopolymers on heterogeneous surfaces. The solution condition is exactly the same as in the previous chapter: we have a transition from high $\mathrm{pH}$ or high temperature to low $\mathrm{pH}$ or low temperature[182]. By designing different copolymers, we can modify the rate of adsorption in order to produce different types of crystals[183]. Adsorption leads to entropy loss, and the equilibrium state is a result of the balance between entropy loss and enthalpy gain from adsorption[184]. Here we have employed the MC simulation method to understand the different phases of the system. The different challenge of applying 
$\mathrm{MC}$ simulation in this chapter from the previous chapter comes from different types of monomer and the way we select the configuration and transitional states[172].

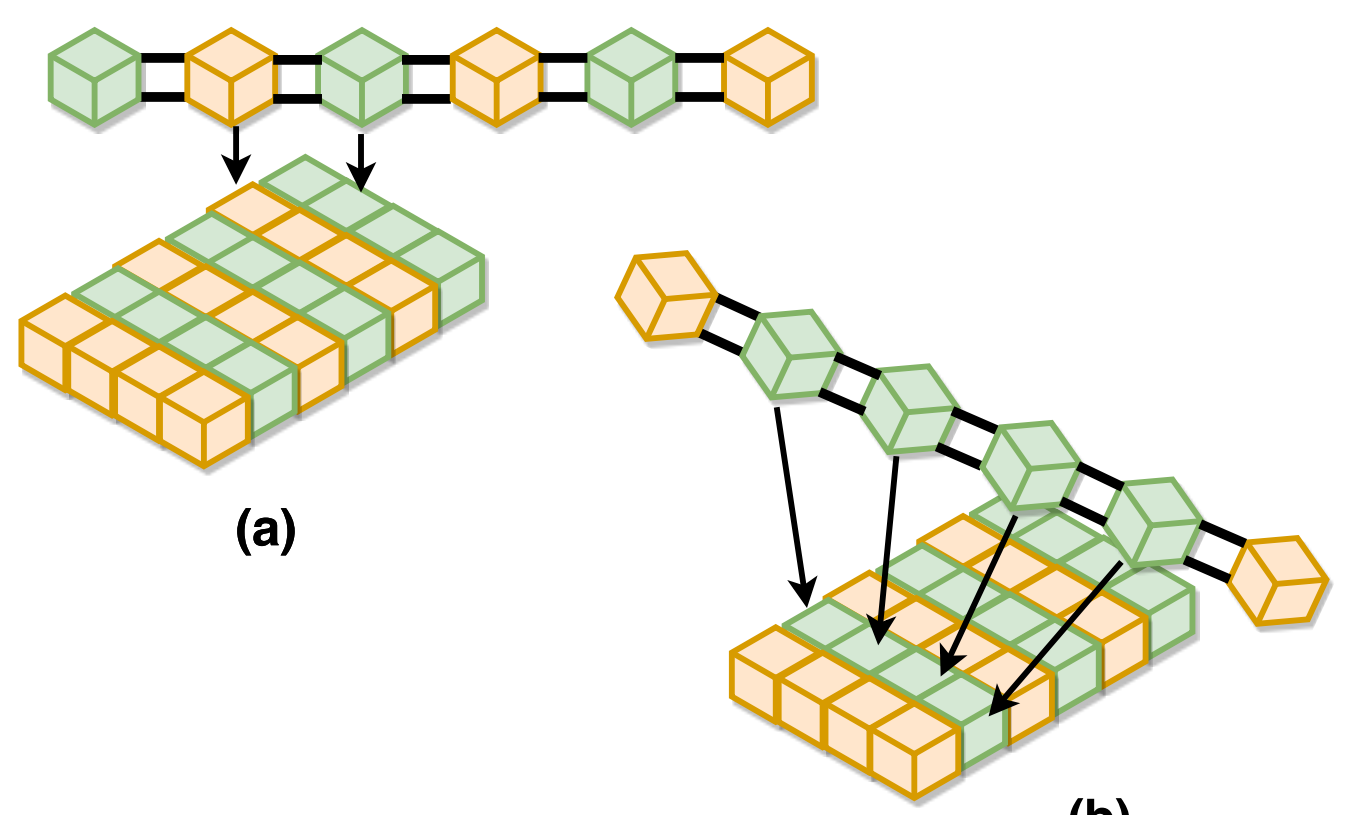

(b)

Figure 6.1: Figure (a) shows diblock copolymer adsorption with the least effective design. In this type of design, the orientation of monomer type B and monomer type $\mathrm{A}$ are such that both can equally distribute over the surface. In Figure (b) monomer type, A and B cannot equally adsorb on the surface and adsorption of type A (green cube) will be preferred to type B (orange cube).

In living systems polymers are flexible and sensitive to change of environment. The most common biological example of such a system is protein molecules adsorption onto cell bodies that needs pattern matching between target cells and proteins[185]. The important elements in this kind of adsorption are the chain length and the sequence of the monomers in the chain, as well as their match to the adsorbing sites, the adsorption strength and the pattern of distribution[186].

Another application of the copolymer is in biopolymers, which are usually random heteropolymer (RHP)[187]. Their multi-functionalities and quench disordered make them interesting for biometric behavior in a definite context. The interest in applying copolymer structure to nucleation is due to modulation at high temperatures. At high temperatures, the copolymeric system exhibits phases where the order parameter is spatially modulated. For example, monomers of type A and type B can never overlap each 
other below the critical temperature; they are energetically attracted at high temperatures. Moreover, they are effectively proven to be surface confined in the nucleation process. Due to the small size of the monomer in the copolymer, the crystallization always experiences higher confinement and more compact morphologies by copolymers. The consequences of confinement by copolymers are reduction of both crystallization and melting temperatures of the crystallization process.

On the other hand, ordered A-alt-B copolymers are of interest for lithographic applications, where copolymers are templates to produce quantum dot arrays [188]. Controlling the domain size through surface interaction is a big challenge in this application.

Furthermore, there are many other applications such as transmembrane signaling pathogen-host interaction, an anti-reflective coating that needs recognition by means of a copolymer chain[189].

The adsorption of copolymer happens at higher temperatures compared to homopolymer systems. In copolymers, the attractive and repulsive interaction between monomer type A and B are different. Therefore the configurational phase space in the copolymer chain is more restricted than homopolymers. As a consequence, due to less fluctuation of conformations, they can find their relaxation states faster.

The important information from the adsorption energy diagram of copolymer systems comes from the diversity of the number of adsorbed monomers type A and B. In crystal growth processes, scientists are interested in controlling the growth of facets with high surface energies to let other facets with lower rates grow more. Therefore in this study, we are interested in designing a polymer with type A + type B that is the most selective in its adsorption on a range of potential surfaces[190]. We try three different patterns of adsorption energies and compare their results together aligned with different domain sizes of islands.

\subsection{Methodology}

Our main focus in this chapter is studying the effect of attractive domain sizes and patterns on adsorbing chains of $\mathrm{A}$ and $\mathrm{B}$ monomers. 
We use the bond fluctuation method (BFM). Our polymer consists of two types of monomer A and B. The chemical nature of the copolymer is such that they can naturally occupy a single unit at high temperature or high $\mathrm{pH}$, whereas at low temperature or low $\mathrm{pH}$ their internal molecular interaction will decrease and monomers will repel each other. As with the previous chapter, we use the expanded ensemble method (EEM) in order to transition from a flexible chain to a rod-like model chain. The surface energy is measured as a unit of energy adsorption which is $\epsilon_{0}=\epsilon=k_{B} T$ and the strength of sites are less than one unit of energy $\left(\epsilon_{A}\right.$ and $\left.\epsilon_{B}<0\right)$.

In order to study the dynamics of the local moves, as in the previous chapter we start with the mechanism of the single move in MC simulation. Since the nature of their fluctuations and conformations are the same, there is no preference for selecting monomer A or B. As a trial conformation we select one monomer randomly and then we check whether the constraint condition of the bond fluctuation method to retain the condition. Next, based on the Rosenbluth condition we weigth both levels of energy states (old $=w_{0}$ and new $=w_{1}$ ). The trial confirmation will be accepted if the random number generated is less than or equal to $\min \left\{1, w_{1} / w_{0}\right\}$. In order to calculate the transition of the system, we first start from the Hamiltonian of the system. We assume that the overlap energy of $N_{o v}(B-B)=N_{o v}(A-A)=N_{o v}(A-B)=k_{B} T$ and the ionic strength $0<\epsilon_{A-A} \neq \epsilon_{B-B} \leq 1$ and $\epsilon_{A-B}=0$. Using this we can write[191]:

$$
H_{t o t}=\left(-N_{A} \epsilon_{A-A}-N_{B} \epsilon_{B-B}+\left(N_{A}+N_{B}\right) \epsilon_{k_{B T}}\right)=U_{i}
$$

the subscript i refers to the current state $i$. The transition probability will be:

$$
W_{i j}=\left(\begin{array}{cc}
w_{a a} & 0 \\
0 & w_{b b}
\end{array}\right)
$$

where $w_{a a}$ and $w_{b b}$ are the probability of adsorption monomer type $\mathrm{A}$ on the site type A with adsorption energy $\epsilon_{A}$ and the probability of adsorption of monomer type $\mathrm{B}$ adsorbing on the site type $\mathrm{B}$ with adsorption strength $\epsilon_{B}$. Thus,

$$
P_{e q}(i) W(i \rightarrow j)=P_{e q}(j) W(j \rightarrow i)
$$

From (Eq. 6.1) and (Eq. 6.3) we find: 


$$
\frac{W(i \rightarrow j)}{W(j \rightarrow i)}=\exp \left(\partial H / k_{B} T\right)=\exp \left(U_{i}-U_{j}\right) / k_{B} T
$$

\subsection{Simulation setup}

We have considered a box of size $24 L * 24 L * 24 L$ where $L$ is the diameter of the monomer, with 24 monomers repeating by A and B units. We design different ways of distributing these two blocks of monomers in the chain. Our goal is to find the strength of interactions between these two units with adsorptive sites which mesh as A and B. Site A only adsorbs unit A with the strength of $0.75 k_{B} T$ and site B only adsorbs unit B with the strength of $0.25 k_{B} T$. We considered three different type of A-alt-B, which are A-B-A-B..., A-A-B-A-A..., A-A-B-B-A-A....

We execute our simulation by distributing one type of chain in each system. We applied periodic boundary conditions on the $\mathrm{x}-\mathrm{y}$ plane and an impenetrable boundary condition on top of the box. For each time step, we selected one monomer randomly and after checking the BFM condition we displaced it to the new cell. If this monomer is close to either end of the chain it can lose its current bond with its nearest neighbors and make a new bond with that end; as a consequence, the previous neighbor will become the new end of the chain. In the homopolymer only the distance of the monomer from the chains end was important. In the copolymer however, the type of monomer is important as well. Since the order of the monomers in a chain is important to us, only the same type of monomers can swap their places, otherwise, the new trial will be rejected. Ultimately we need to calculate the Rosenbluth weighting function based on the energy level of the current place and the new place (see section 5.4.1.1).

As in the previous chapter, we would like to find different phases of the system and the temperature of each phase transition. Here we show only one case of copolymer chain adsorption AABAA on striped pattern to become familiar with the general idea of phase transitions of diblock copolymer adsorption onto a striped heterogeneous surface. We assume the width of each site is $\mathrm{L}=2$ and the length is equal to the length of the box $\mathrm{D}=24$. Three major phase transitions have been shown and in general four phases are 


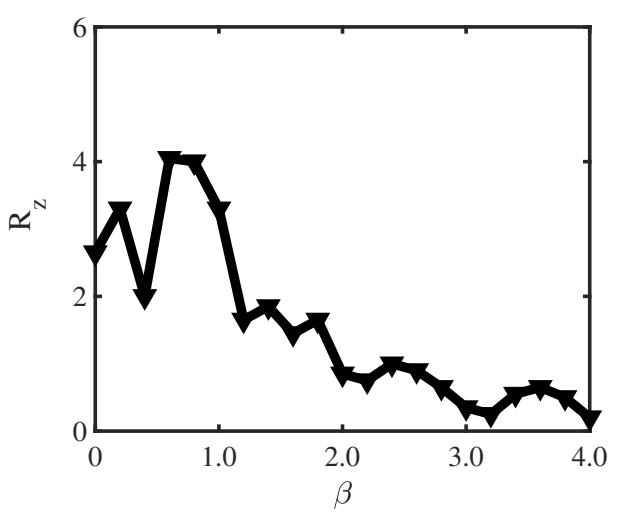

(A) Centre of mass in the $\mathrm{z}$ direction for striped pattern, $L=2$, for copolymer chain

$\mathrm{H}$

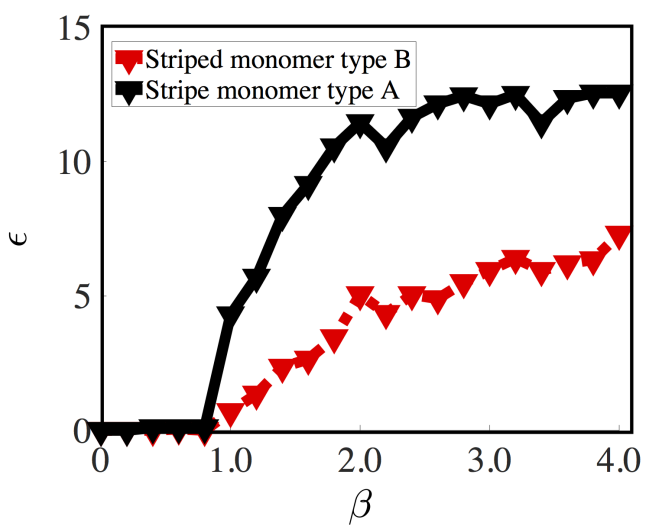

(B) Adsorption enery for striped pattern with active site $L=2$, for monomer type A and type B in copolymer chain AABAA.

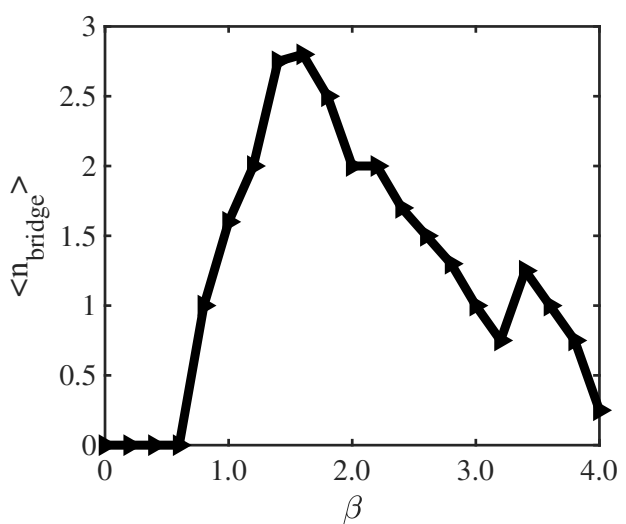

(c) Average number of bridges of striped pattern with active sites $L=2$. For only monomer type A with $\epsilon_{A}=0.75$

Figure 6.2: This figure shows (A) summary of three important phases that occur during simulation. In the first figure, line drop from maximum to minimum shows the transition from fully crumpled to the expanded state. In this figure the type of monomer is not really important, therefore we did not consider them separately. Figure (B) shows the adsorption energies of two types of monomers. With this information we can find the phase transition from desorption to adsorption states once first a few monomer starts to become adsorbed. Figure $(\mathrm{C})$ show the pattern recognition by monomer type A that the strength of adsorption is bigger than type B. Once the total number of bridges tend to zero, we consider copolymer has recognized the surface.

studied: phase transitions occur from fully crumpled to expanded state, from desorbed state to adsorption state and from being fully adsorbed to fully recognize the surface. 


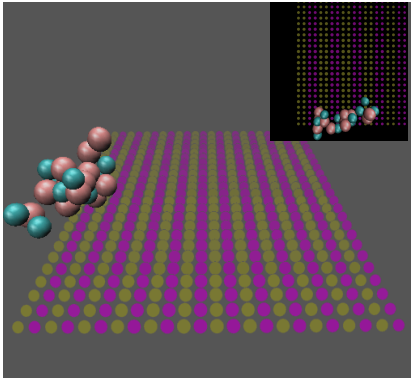

(a)

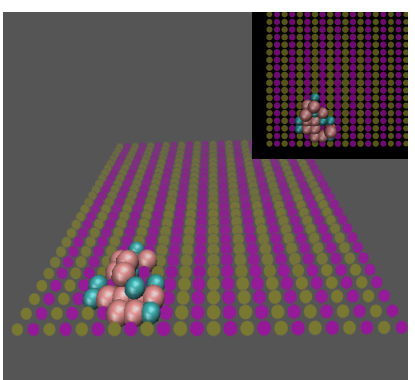

(d)

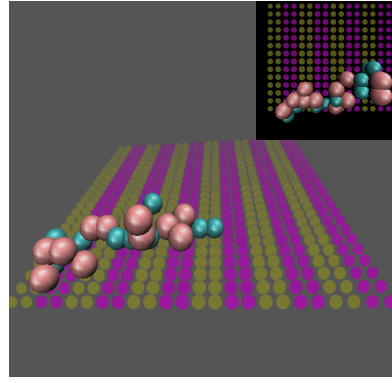

(b)

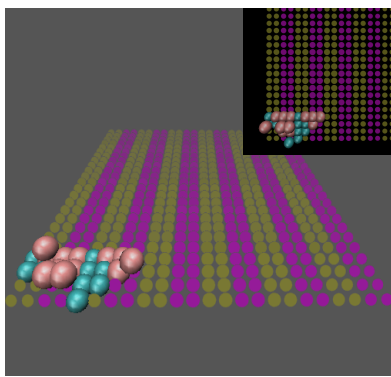

(e)

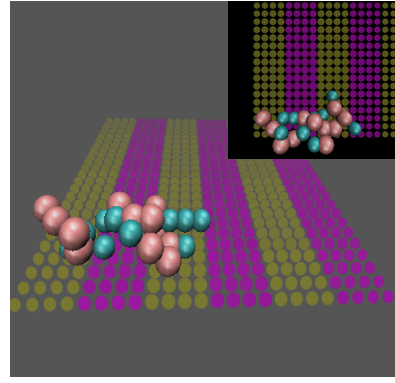

(c)

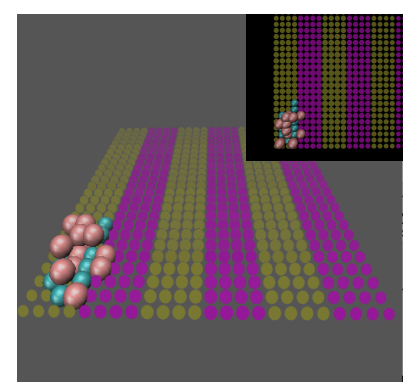

(f)

(A) snapshots of AABB chain adsorption on striped pattern.Figure a to c shows the snapshots of high temperature $(\beta=0.0)$, whereas figure $\mathrm{d}$ to $\mathrm{f}$ shows lower temperature adsorption correspond to the size of islands $(\beta=0.8,0.7,0.65)$

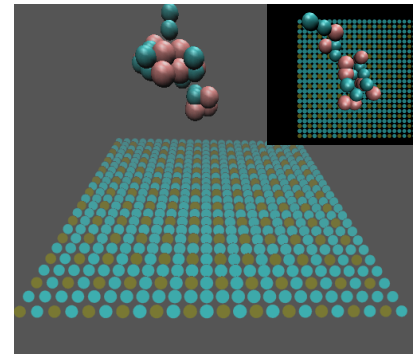

(a)

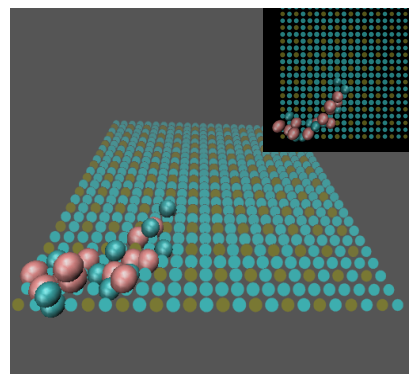

(d)

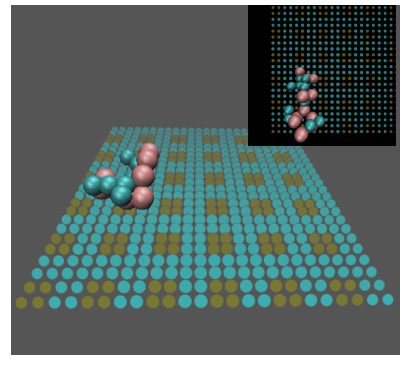

(b)

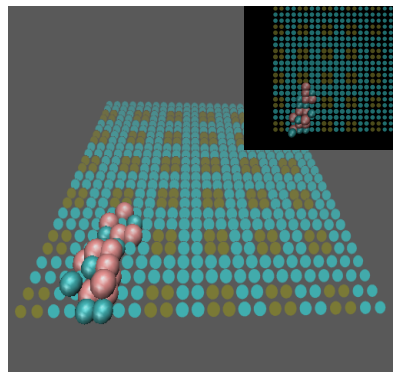

(e)

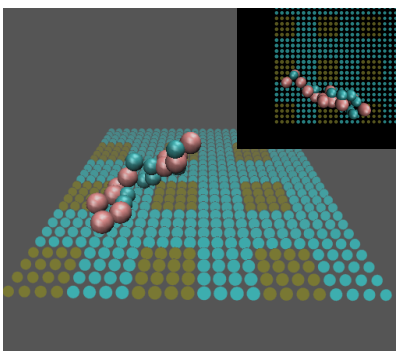

(c)

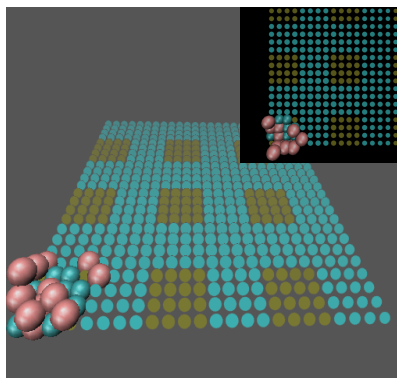

(f)

(B) snapshots of AABB chain adsorption on checked pattern. Figure a to $\mathrm{c}$ shows the snapshots of high temperature $(\beta=0.0)$, whereas $\mathrm{d}$ to $\mathrm{f}$ shows lower temperature adsorption $(\beta=1.0,0.9,0.8)$ correspond to the size of islands $(\mathrm{L}=1,2,4)$

Figure 6.3: The snapshots of two different patterns at different temperatures with the size of domain $=1,2,4$ 
As in the previous chapter, in order to understand the phase transition from fully crumpled polymer to extended polymer we need to calculate the centre of mass of the copolymer chain. Here we calculate the center of mass of the polymer in the $\mathrm{z}$ direction in order to understand the elasticity of the chain and its phase transition within a range of temperature Figure. 6.6 and Figure. 6.7 show the evolution of the center of mass as a function of temperature for different patterns on the surface. From the snapshots of simulation we see the adsorption of different types of the copolymer on patterned surfaces: Striped, Checked and Random. In this chapter due to the heterogeneity of the surface and polymer chain, we expect more complex process comparing to the previous chapter. However, in some cases monomer type A prefer to only adsorb on the certain sites and type $\mathrm{B}$ on other sites. This property can facilitate the adsorption process if the periodicity of the monomers is the same as the periodicity of the adsorption site. As a consequence, the phase temperature for some cases occur earlier than homopolymer chain and for some other cases because the periodicity of the chain is different from the periodicity of the surface, the adsorption process becomes difficult. 


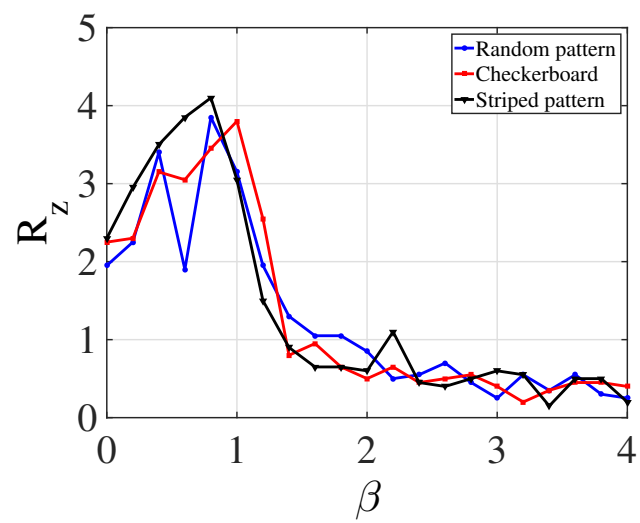

(A) Centre of mass of ABAB chain in the $\mathrm{z}$ direction for active site $\mathrm{L}=1$

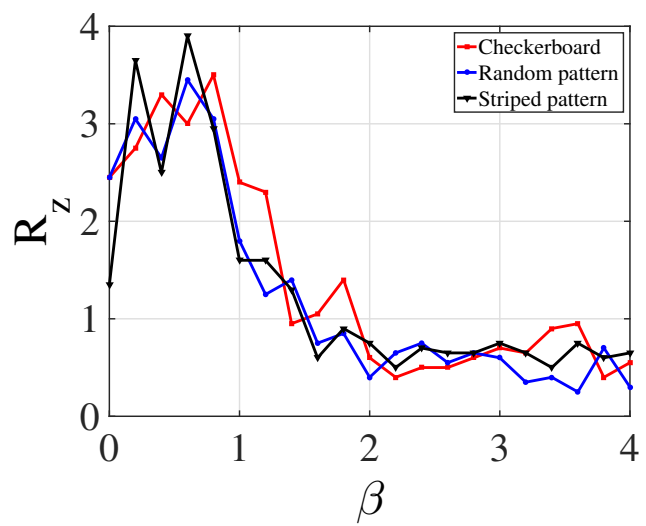

(C) Centre of mass of ABAB chain in the $z$ direction for active site $\mathrm{L}=4$

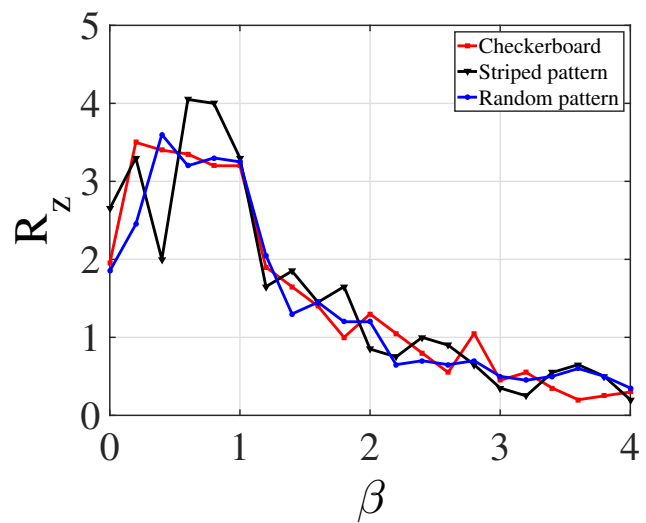

(E) Centre of mass of AABB chain in the $\mathrm{z}$ direction for active site $\mathrm{L}=2$

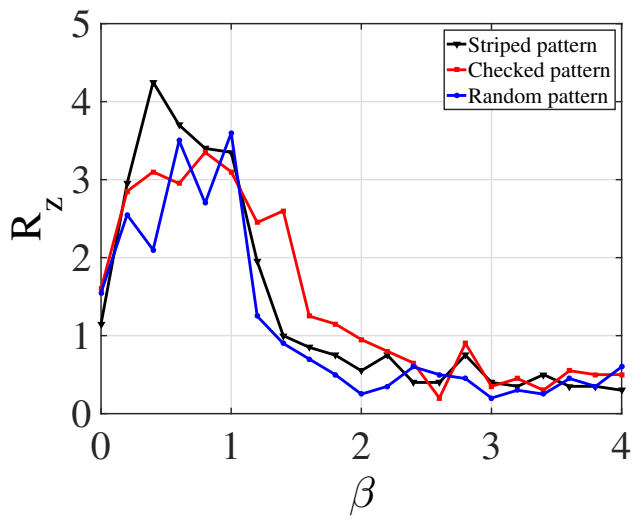

(B) Centre of mass of $\mathrm{ABAB}$ chain in the $\mathrm{z}$ direction for active site $\mathrm{L}=2$

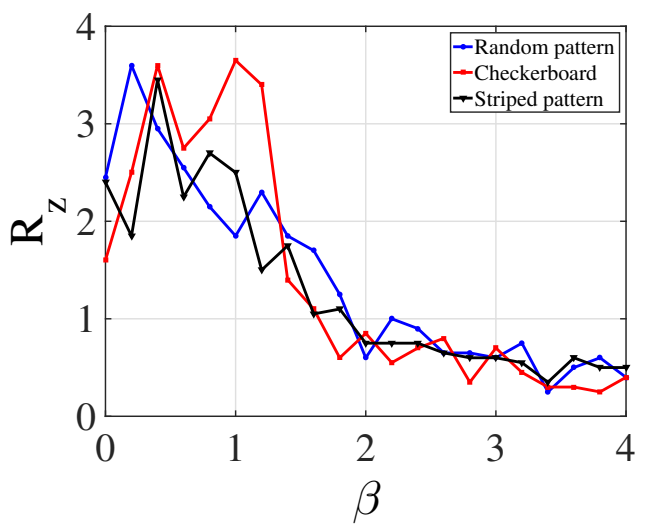

(D) Centre of mass of AABB chain in the $\mathrm{z}$ direction for active site $\mathrm{L}=1$

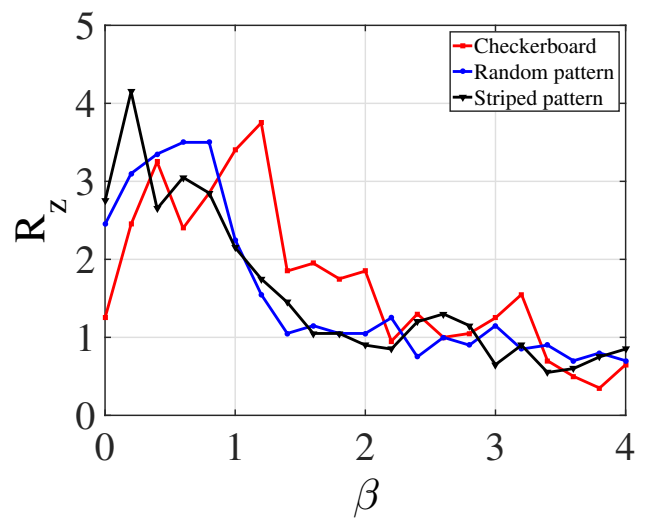

(F) Centre of mass of AABB chain in the $\mathrm{z}$ direction for active site $\mathrm{L}=4$

FigurE 6.6: These figures show the length of $\mathrm{R}$ in the $\mathrm{z}$ direction. First three pictures are for ABAB chain. The most chaotic pattern for the surface is random. On the other hand, the maximum point of each pattern is different from one another. The maximum point of $\mathrm{Rz}$ even lies to lower temperature for the chain AABAA. 


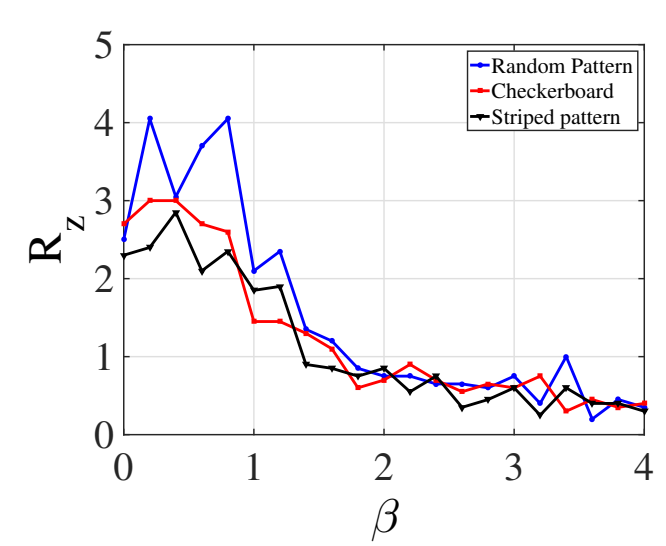

(A) Centre of mass of AABAA chain in the $\mathrm{z}$ direction for active site $\mathrm{L}=1$

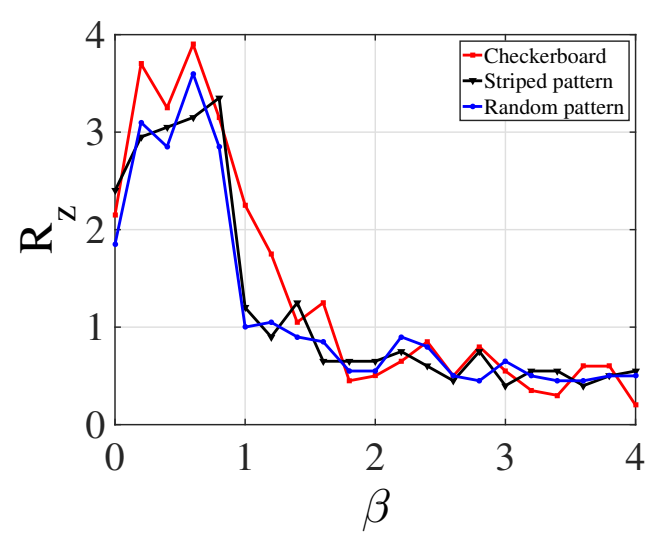

(B) Centre of mass of AABAA chain in the $\mathrm{z}$ direction for active site $\mathrm{L}=2$

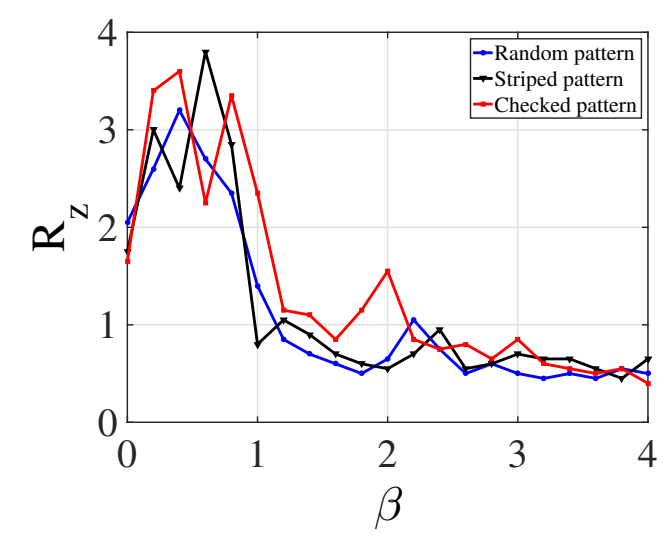

(C) Centre of mass of AABAA chain in the $\mathrm{z}$ direction for active site $\mathrm{L}=4$

Figure 6.7: The average of centre of mass for different pattern and different size of active sites for one type of chain. The maximum length of $\mathrm{R}$ in $\mathrm{z}$ direction for this pattern of chain is somewhere between ABAB and AABAA.

\subsubsection{Adsorption Energy}

As mentioned in (section. 6), in order to find a desirable rate for growing a crystal we need to alter the pattern of the monomers within a copolymer chain. In order to design the best patterns of monomers in the chain we need to have good information about the different surface energies of the surface. Here we assume there are only two types of facets on the surface. Different types of state can be studied. The nonideal case scenario would be when type A and type B are adsorbing the same amount of monomers at equilibrium state or equivalently the same rate. We studied the effect of distribution of monomers at a given potential energy of the surface[172]. 
We calculated three different types of chain for adsorption onto three different patterns (striped, checkerboard and random).

We start from (Figure. 6.8) which shows the chain type ABAB, so the number of monomer type $\mathrm{A}$ and $\mathrm{B}$ should be exactly the same. In (Figure. 6.8a) since the periodicity of the chain is the same as the periodicity of the island sites, we have the maximum number of adsorption for both types of $\mathrm{A}$ and $\mathrm{B}$, which is not advantageous for modifying the crystal growth rate. Indeed with this distribution it is similar to a homo polymer chain onto a homogeneous adsorption surfaces. Therefore, we see if we want to grow a crystal with a specific pattern that matching the orientation of the block monomers with the same strength of attractive sites on the surface might not be that ideal. In order to create heterogeneity in crystal growth we need to apply a chain that favors one pattern and against to another. One way of doing this is to design a new pattern of orientation of monomers within a chain. We can design a chain such that one type of monomer becomes fully adsorbed on one type of site and another type stay none attracted from the surface and let that surface grow with solution. As we increased the size of the absorbative patches, the diversity of adsorption of the two different types of monomers will increase. However, the distinction between different patterns become less significant. As a result, we are interested in the distinctive number of adsorption of different monomers and different patterns. The ideal case is shown in figure( $6.9 \mathrm{~b})$. As we can see there is a significant difference between monomer type A and B and there are also different responses to different patterns. 


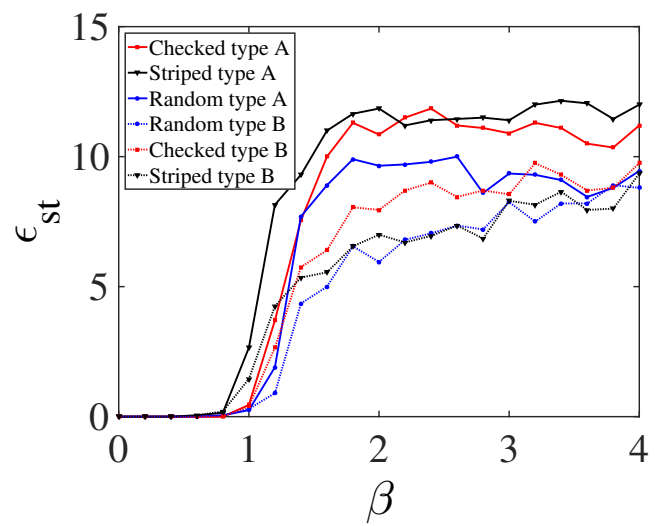

(A) Adsorption energy of monomers type A with the strength of adsorption $\epsilon_{A}=0.75$ and monomers type B with the strength of adsorption $\epsilon_{B}=0.25$ with the length of adsorption $L=1$, for the design of $A B A B$

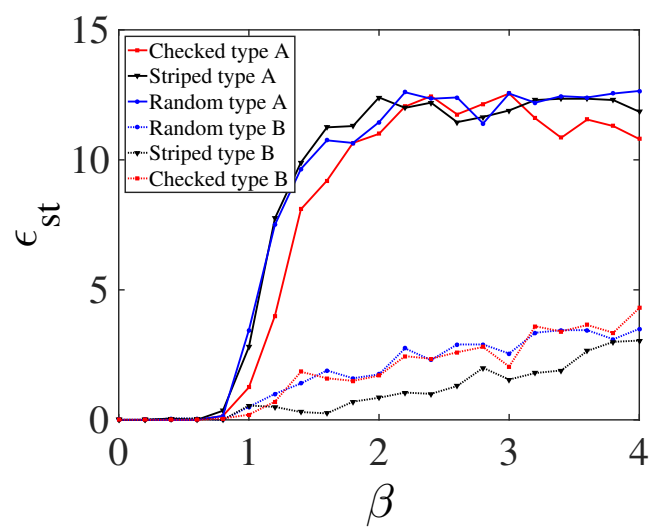

(C) Adsorption energy of monomers type A with the strength of adsorption $\epsilon_{A}=0.75$ and monomers type B with the strength of adsorption $\epsilon_{B}=0.25$ with the length of adsorption $L=4$, for the design of $\mathrm{ABAB}$

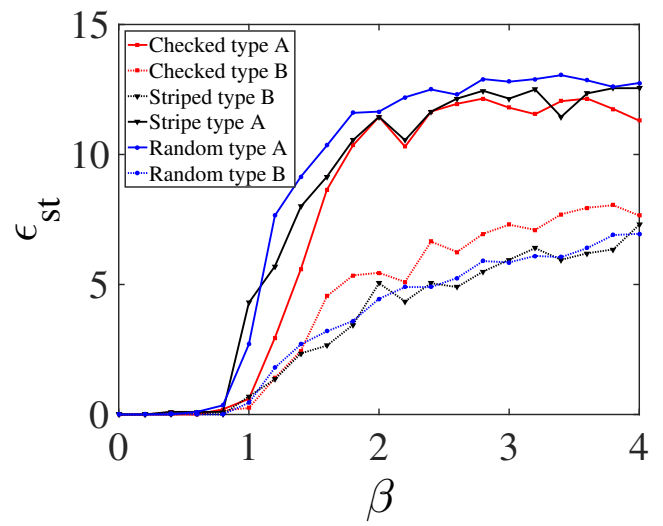

(E) Adsorption energy of monomers type A with the strength of adsorption $\epsilon_{A}=0.75$ and monomers type $\mathrm{B}$ with the strength of adsorption $\epsilon_{B}=0.25$ with the length of adsorption $L=2$, for the design of AABB

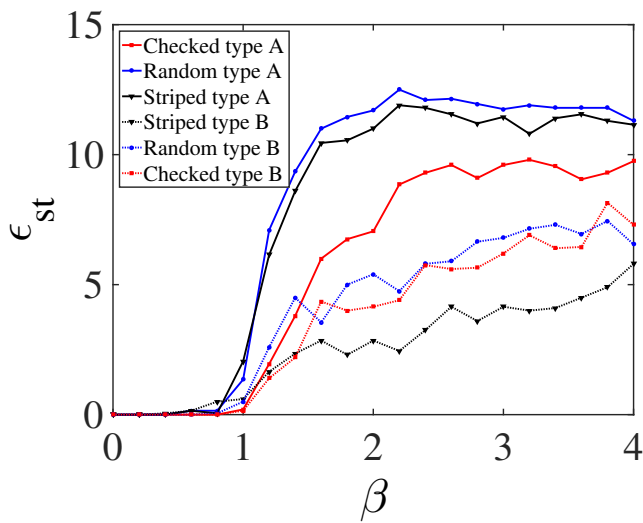

(B) Adsorption energy of monomers type A with the strength of adsorption $\epsilon_{A}=0.75$ and monomers type B with the strength of adsorption $\epsilon_{B}=0.25$ with the length of adsorption $L=2$, for the design of $\mathrm{ABAB}$

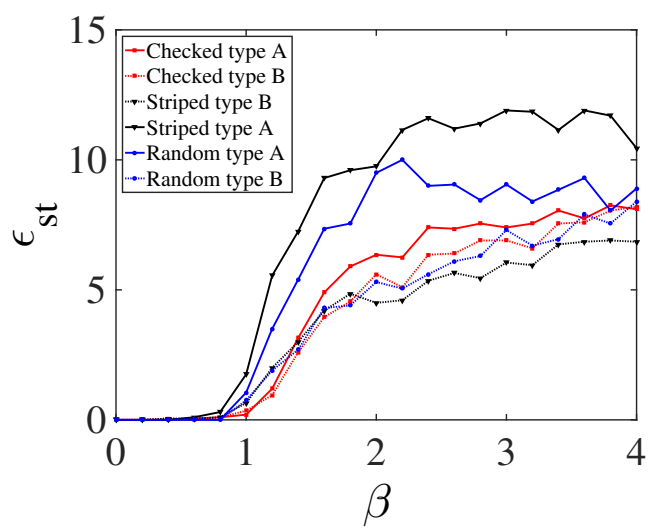

(D) Adsorption energy of monomers type A with the strength of adsorption $\epsilon_{A}=0.75$ and monomers type $\mathrm{B}$ with the strength of adsorption $\epsilon_{B}=0.25$ with the length of adsorption $L=1$, for the design of AABB

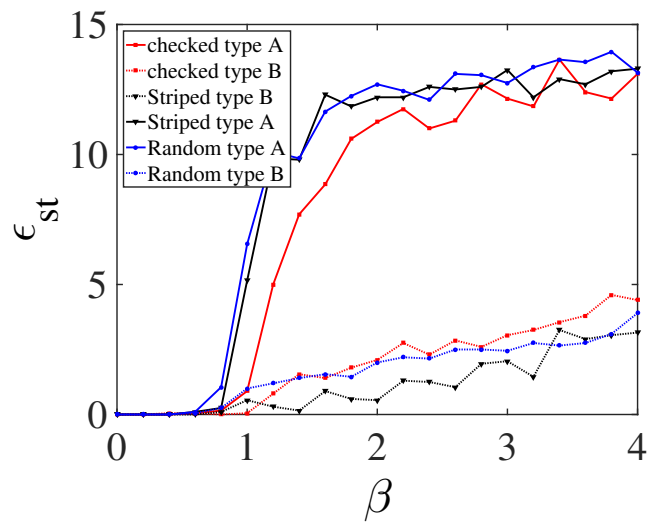

(F) Adsorption energy of monomers type A with the strength of adsorption $\epsilon_{A}=0.75$ and monomers type $\mathrm{B}$ with the strength of adsorption $\epsilon_{B}=0.25$ with the length of adsorption $L=4$, for the design of AABB

FiguRE 6.8: Total number of adsorbed monomer of AABBAA and ABAB over a certain range of $\beta$ for different size of islands 


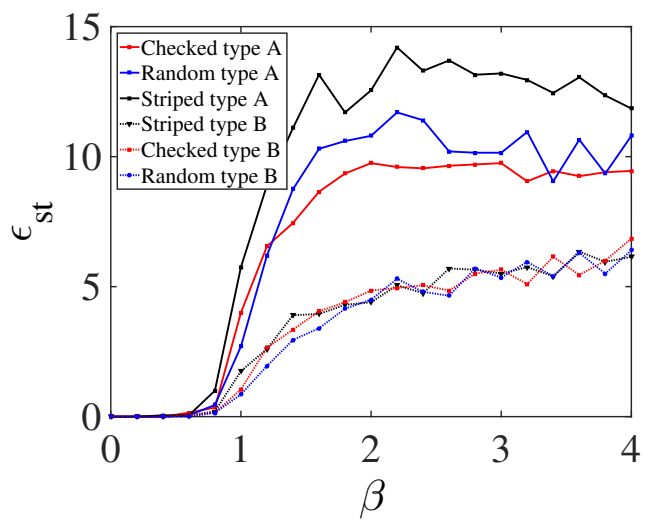

(A) Adsorption energy of monomers type A with the strength of adsorption $\epsilon_{A}=0.75$ and monomers type $\mathrm{B}$ with the strength of adsorption $\epsilon_{B}=0.25$ with the length of adsorption $\mathrm{L}=1$, for the design of $\mathrm{AAB}$

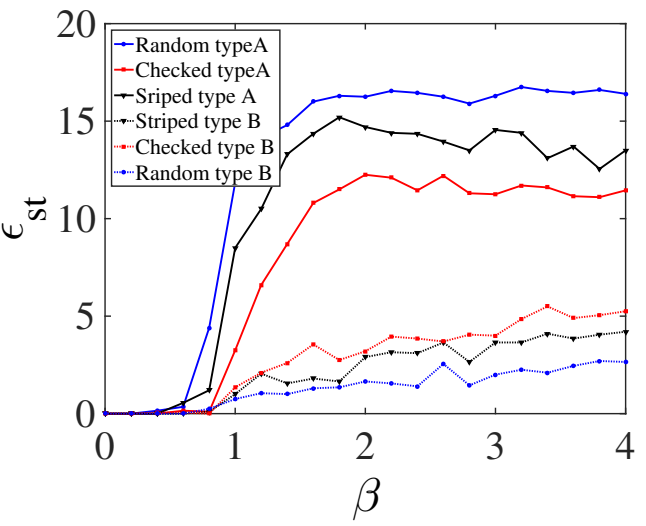

(B) Adsorption energy of monomers type A with the strength of adsorption $\epsilon_{A}=0.75$ and monomers type B with the strength of adsorption $\epsilon_{B}=0.25$ with the length of adsorption $\mathrm{L}=2$, for the design of $\mathrm{AAB}$

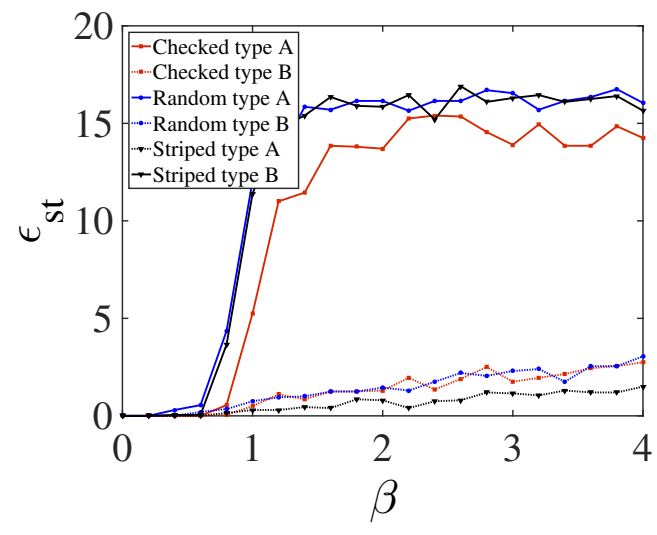

(C) Adsorption energy of monomers type A with the strength of adsorption $\epsilon_{A}=0.75$ and monomers type $\mathrm{B}$ with the strength of adsorption $\epsilon_{B}=0.25$ with the length of adsorption $\mathrm{L}=4$, for the design of $\mathrm{AAB}$

FiguRE 6.9: Total number of adsorbed monomer of AABAA over the certain range of $\beta$ for different size of islands

\subsubsection{Pattern Recognition}

The pattern recognition state is important in many copolymer systems. Understanding the temperature or $\mathrm{pH}$ at which a polymer matches monomers with adsorption sites is an important part of this analysis. Once the homopolymer chain fully recognizes a pattern and becomes adsorbed on one type of site we call the pattern recognition has occurred. Whereas in copolymer systems, due to the heterogeneity of the chain and surface, once one type of monomers adsorbed on certain sites, and other types of monomers 
will have difficulty to become adsorbed on the surface due to repulsion between different types of monomer, which this lead to special nucleation on the surface.

(Figure. 6.10) shows the results from simulation of adsorption on two patterns. Here we only consider one type of monomer and we show the average number of bridges within a certain range of temperature. Different dashed lines represent the different sizes of adsorption regions. Obviously, small sizes limit the adsorption, so they have a larger number of bridges, whereas large sites have a small number of bridges. In both subfigures, we focused on the strong type of adsorption, which is $\epsilon_{A}=0.75$. If we compare the stripe pattern at $\beta=4$ for $L=2$ with $\beta=4$ for $L=2$ on a checkerboard pattern we see the system still has not fully recognized the pattern, which is the result of the high entropy of the polymer adsorbed on the checkerboard pattern.

\subsubsection{Phase diagram}

Figure 6.12 and 6.12 are the the phase diagrams of the most selective design of copolymer AABAA onto striped and checkerboard pattern. The temperature and length of attractive sites as order parameters play key roles in the phase transitions. The phase diagram of copolymer system with heterogeneous attractive sites consists of several phases: first, at high temperatures the level of entropy of the system is such that not a single monomer can adsorb on the surface. Due to the heterogeneous pattern in a chain, the loss of entropy occurs faster than the homogenous chain and a few monomers with strong adsorption strength start to become adsorbed on the surface and they will drag off the rest of the chain onto the surface. However, the periodicity and the size of the island will restrict monomers to only occupy the right adsorption site. Therefore most of the time, the full adsorption state, and recognition states will at lower temperatures than homopolymer adsorption. Technically when there is no bridge between identical monomers in adsorbed sites, the chain has recognized the surface. However, the one that we are interested in is the contrast adsorption between monomer type A and type B. In another words, one type must overcome the other type in terms of gaining the adsorption energy of state.

Therefore here we selected AABAA as the most distinctive copolymer chain in terms of adsorption onto different types of sites. Figure. 6.5,6.4,6.3 are the examples of different 
orientation of diblock compolymer chain for ABAB, AABAA, and AABBAA chain. These figures show the number of adsorbed monomers of copolymer chain under the same thermodynamics condition and same size of adsorption sites.

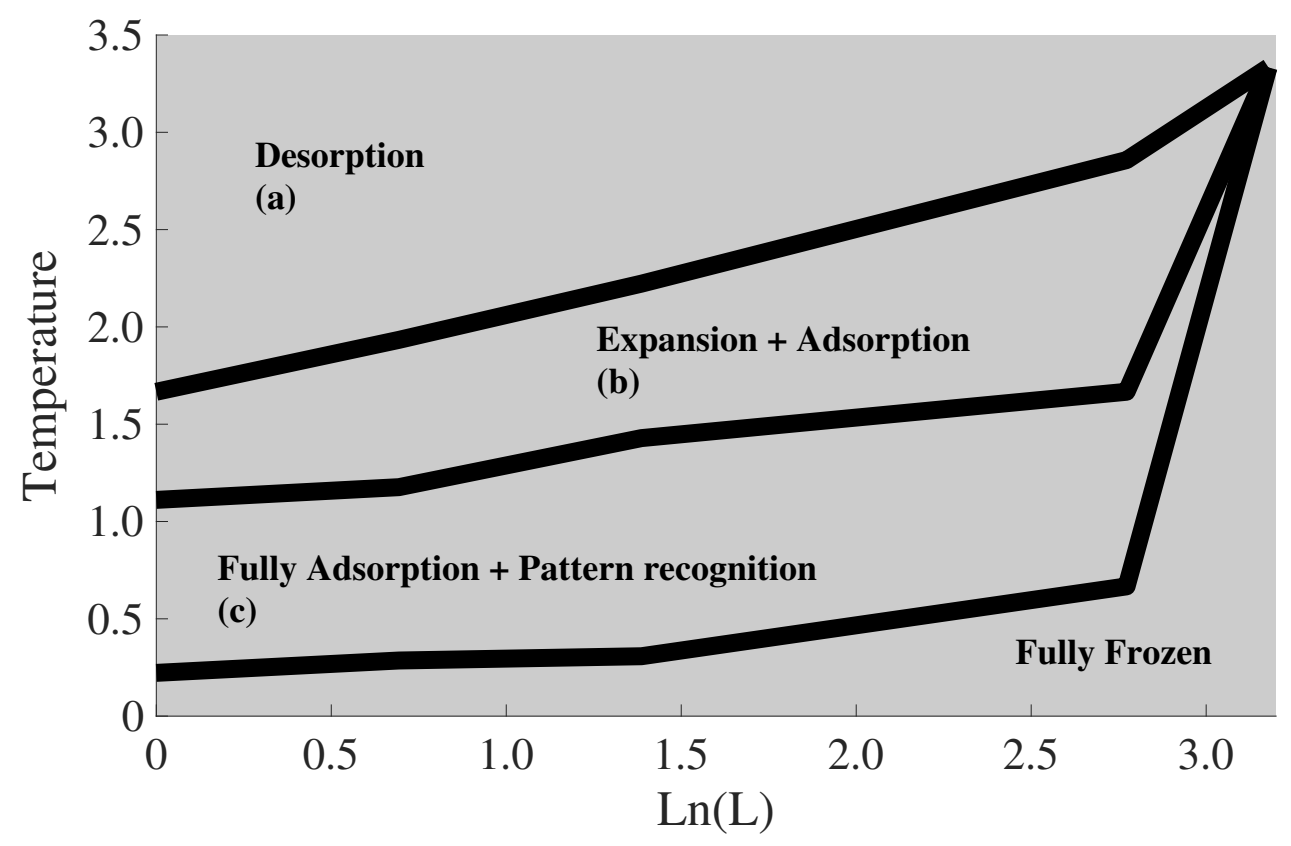

Figure 6.11: This figure shows the phase diagram of the most selective design of copolymer AABAA in striped pattern as a function of length of sites and temperature. Phase (a) system is at very high temperature, phase (b) system starts to become expanded and adsorbed on the surface, phase (c) chain is become fully adsorbed). The width of the lines show that the boarder between two regions is not very sharp and it changes from case to case. Therefore, we cannot separate two phases with a certain point 


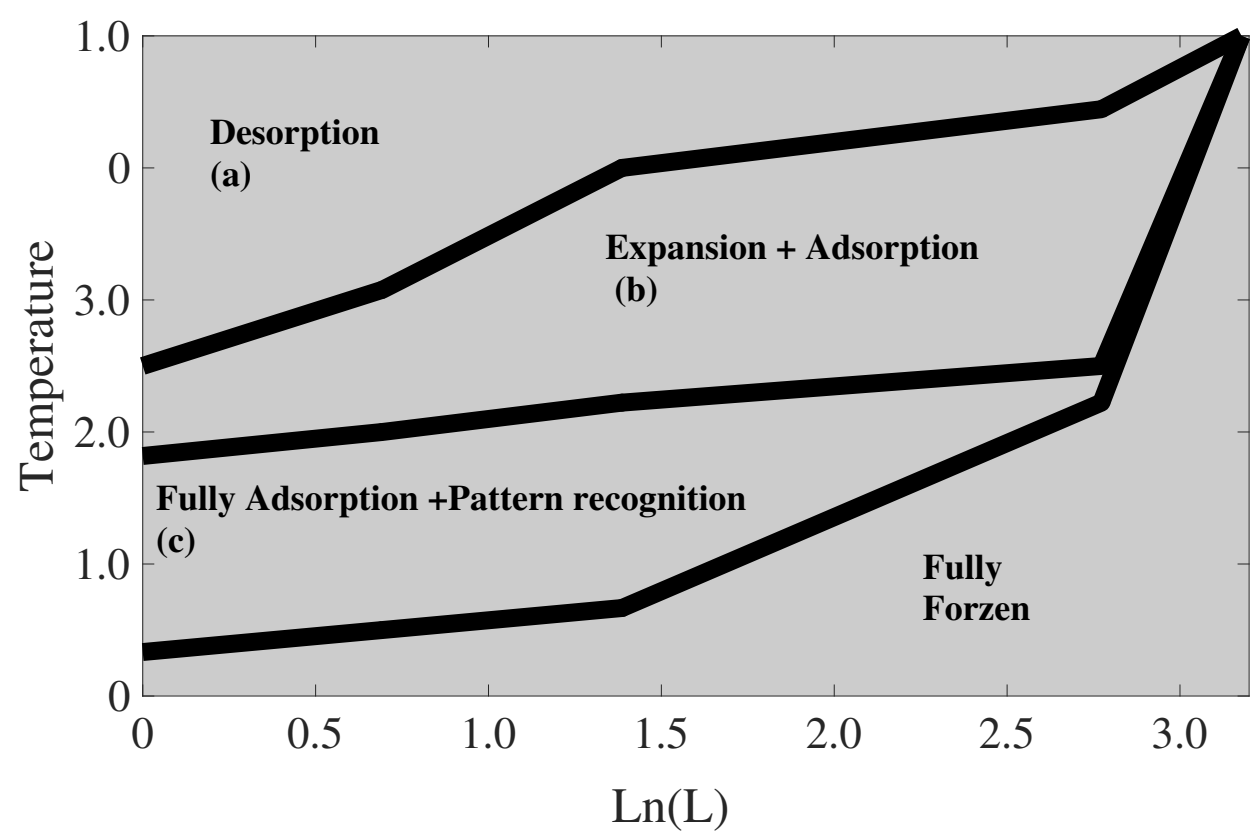

Figure 6.12: This figure shows the phase diagram of the most selective design of copolymer AABAA in checkerboard pattern as a function of length of sites and temperature. Phase (a) system is at very high temperature, phase (b) system starts to become expanded and adsorbed on the surface, phase (c) chain is become fully adsorbed. The width of the lines show that the boarder between two regions is not very sharp and it changes from case to case. Therefore, we cannot separate two phases with a certain point

\subsection{Conclusion}

We have applied the Monte Carlo simulation method with the Bond-Fluctuation model and an expanded ensemble method to study copolymer chain adsorption on a flat chemically heterogeneous surface. The main objective of this study is understanding the size effect of heterogeneous attractive sites and their distribution patterns on effective adsorption of different types of copolymer chain. Our result revealed that different blocks of copolymer orientation can alter the adsorption temperature. With this information, one can design the new, desired crystal structure by optimizing a polymer chain to favor particular crystal surfaces. Since examining the different nanostructure of the surface by experimenting is difficult and sometimes leads to wasting a high amount of material, by this computational method one could first design the best distribution of copolymer for a specific pattern then apply the result to an experiment. As a consequence, it means that are may be able to control the rate of crystallization effectively. By designing a 
polymer that will preferentially adsorb on particular surfaces. We have examined only three different blocks of A-Alt-B copolymer chain. This result showed that not only is there a high distinction between adsorption of the different segments but also comparing different patterns of the surface, there is quite a significant gap between striped, random and checkerboard, and therefore, the distribution matters as well.

However, we only considered specific patterns of the surface and the copolymer chain. There are different ways of distributing monomers in the chain. Designing the best copolymer chain based on the pattern of the surface through MC simulation helps us to pick up the efficient copolymer chain instead of trying different chain in the experiment. 


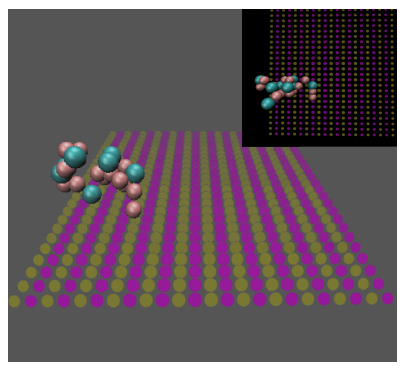

(a)

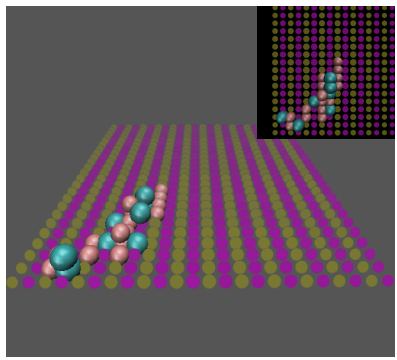

(d)

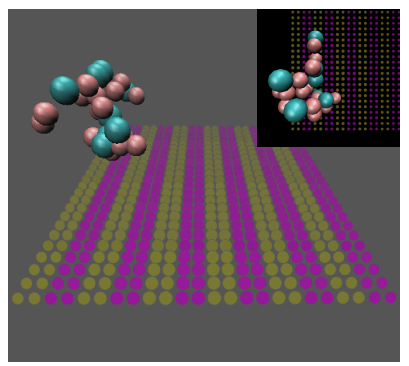

(b)

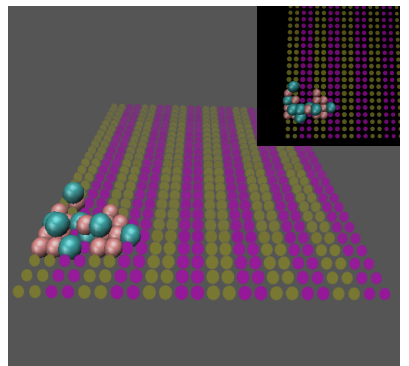

(e)

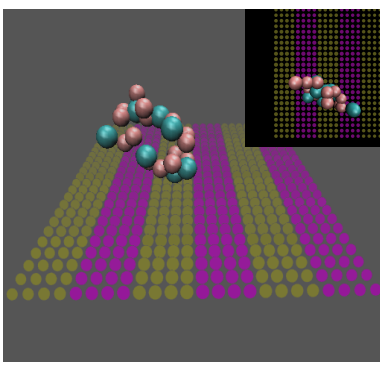

(c)

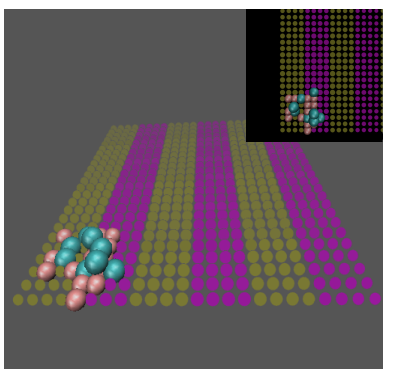

(f)

(A) snapshots of AABAA chain adsorption on striped pattern. Figure a to c shows the snapshots of high temperature $(\beta=0.0)$, whereas figure $\mathrm{d}$ to f shows lower temperature adsorption $(\beta=1.0,0.9,0.8)$ correspond to the size of islands $(\mathrm{L}=1,2,4)$

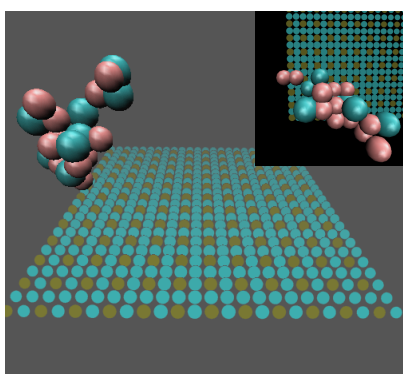

(a)

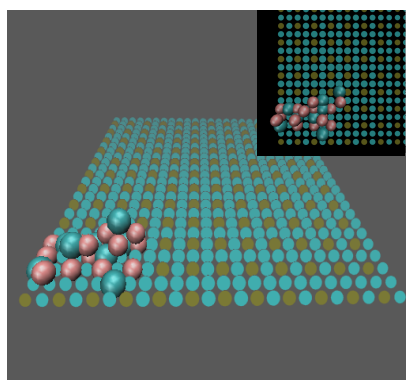

(d)

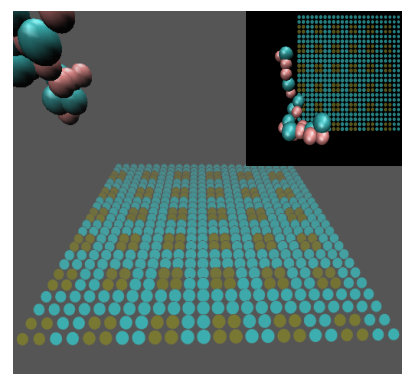

(b)

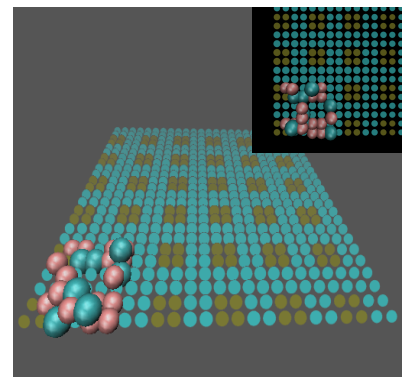

(e)

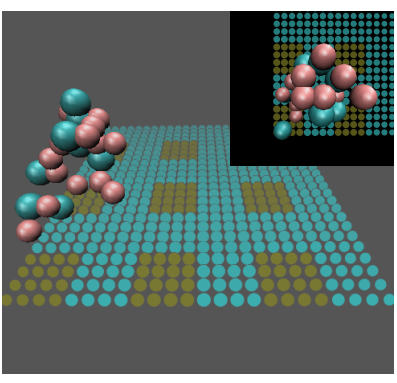

(c)

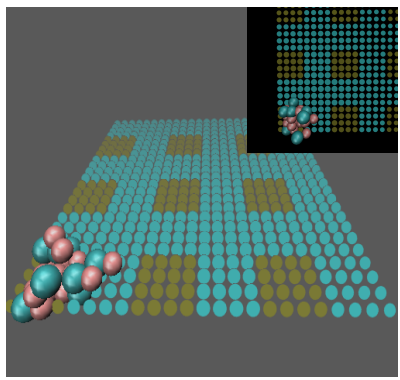

(f)

(B) snapshots of AABAA chain adsorption on checked pattern. Figure a to c shows the snapshots of high temperatures, whereas figure $d$ to $f$ shows lower temperatures adsorption correspond to the size of islands $(\beta=0.8,0.65,0.5)$

Figure 6.4: The snapshots of two different pattern at different temperatures with the size of domain $=1,2,4$ 

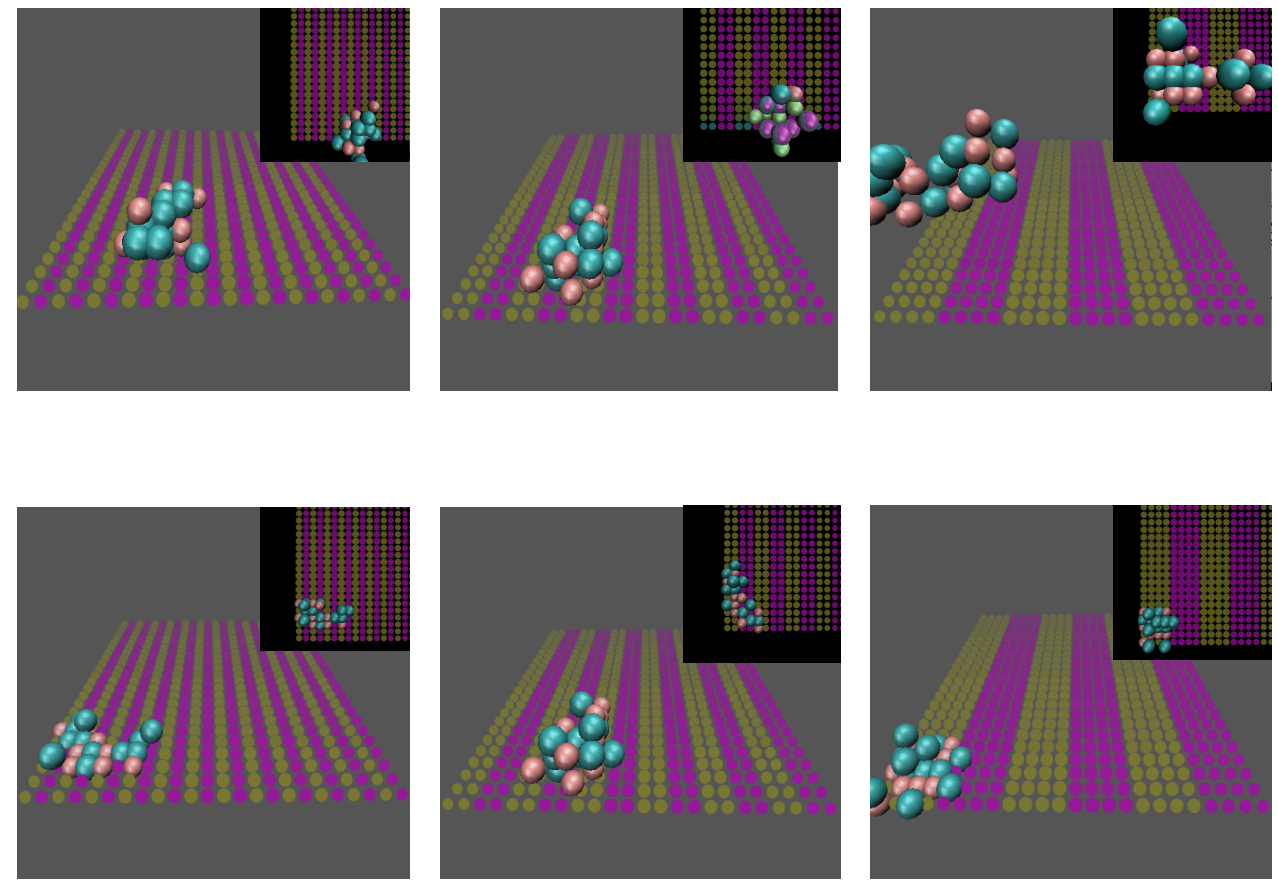

(A) snapshots of ABAB chain adsorption on striped pattern. Figure a:c shows the snapshots of high temperatures $(\beta=0.0)$, whereas d:f shows lower temperatures adsorption correspond to the size of islands $(\beta=0.75,0.65,0.5)$

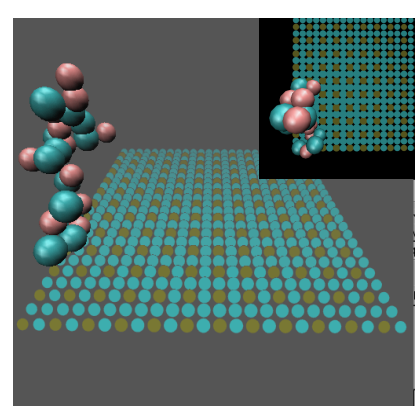

(a)

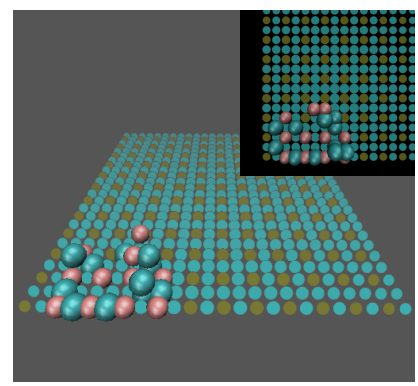

(d)

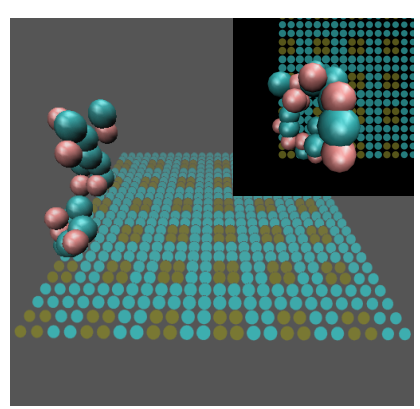

(b)

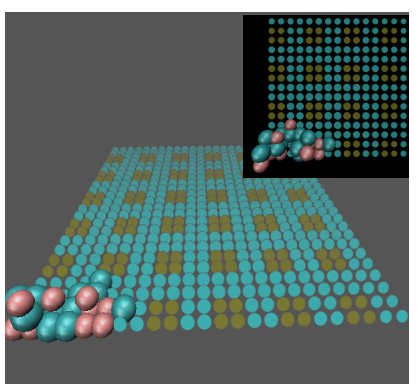

(e)

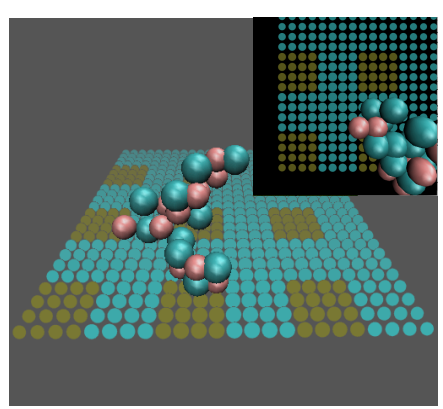

(c)

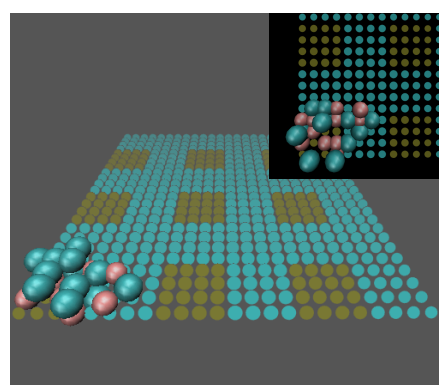

(f)

(B) snapshots of ABAB chain adsorption on checked pattern. Figure a:c shows the snapshots of high temperatures $(\beta=0.0)$, whereas d:f shows lower temperatures adsorption correspond to the size of islands $(\beta=1.0,0.9,0.75)$

FiguRE 6.5: The snapshots of two different pattern at different temperatures with the size of domain $=2$ 


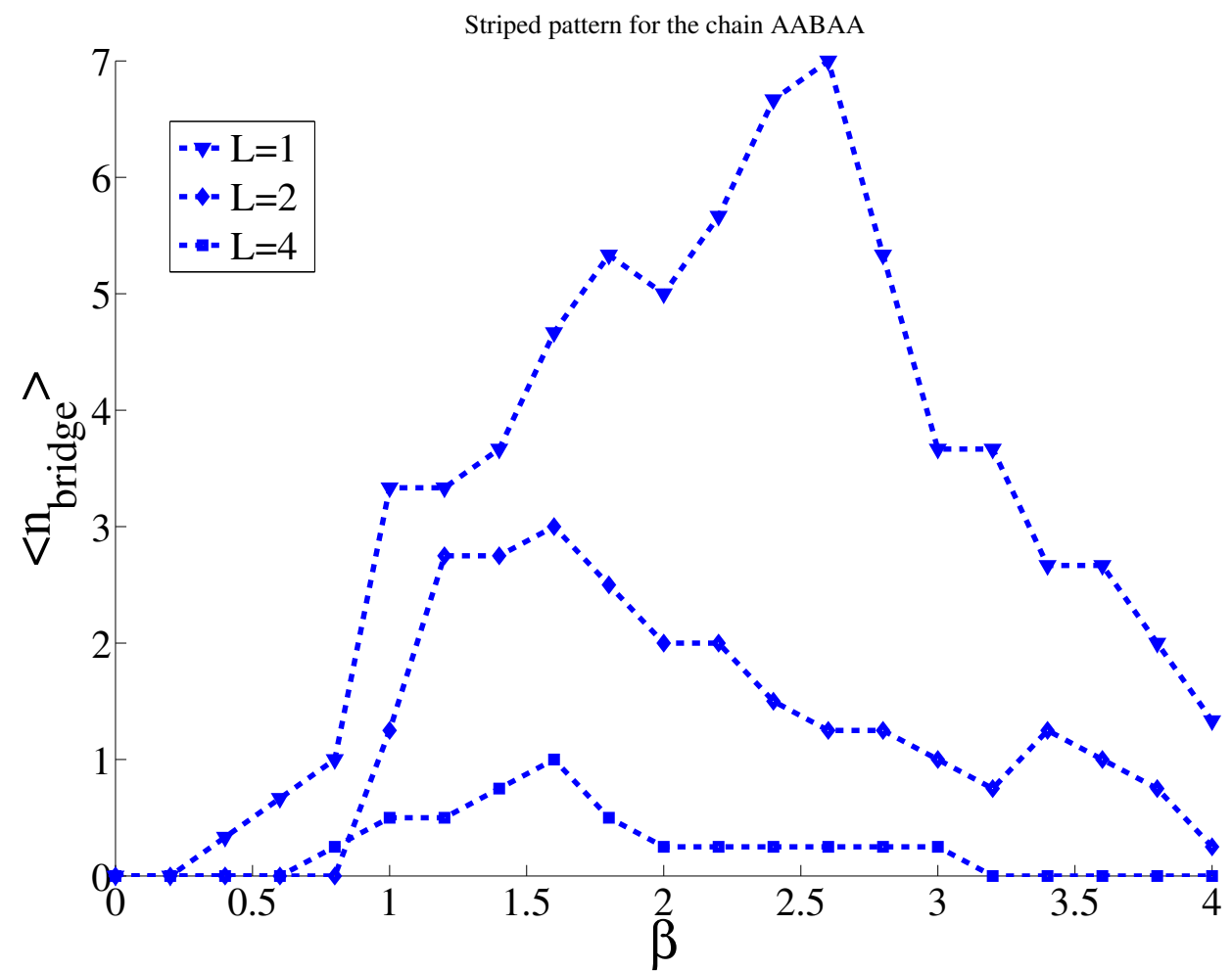

(A) Average number of bridges for striped pattern. Different lines shows different size of islands

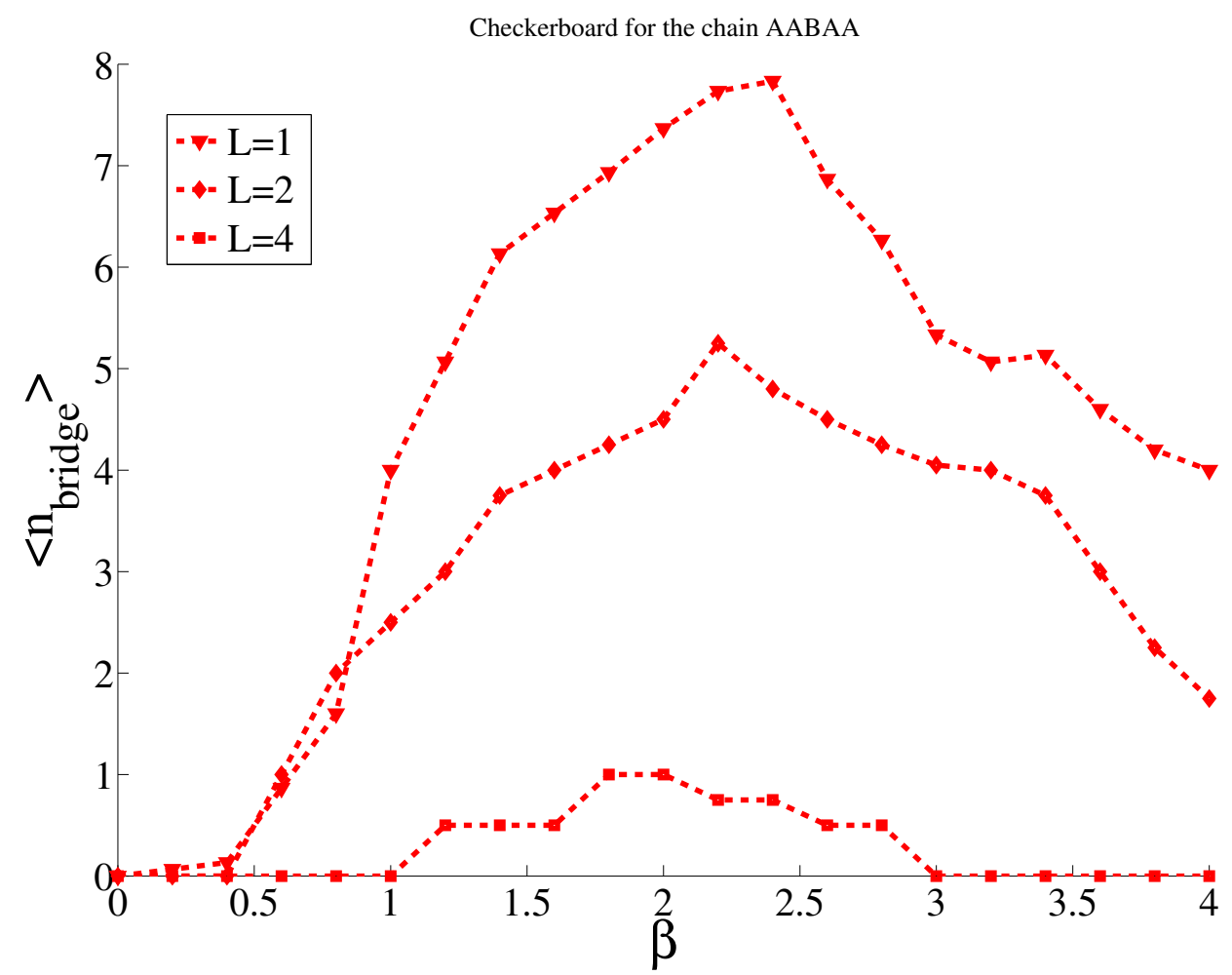

(B) Average number of bridges for checkerboard surface. Different lines shows different size of islands

FiguRE 6.10: The average number of bridges comparison between two patterns: striped and checkerboard 


\section{Chapter 7}

\section{Conclusion}

Different morphologies of the same compound are the result of the surface energy difference of the crystal faces, and the growth mechanisms which cause some faces to grow faster than others. There are different ways of controlling crystal growth. Most of the methods at microscale modify the growth rate. Studying the growth rate in experiment is not easy since distinguishing between different phases in short time intervals can sometimes be inaccurate. However, by computational methods one can simulate the whole process in a virtual space. Here we applied the Kinetic Monte Carlo method to find the tiny events that occur during crystallization process and help to modify the rate of crystal growth. Two approaches have been followed in this thesis: one is the growth of crystal with different surface energies, and the other is through free polymer chains.

We consider a single crystal with different crystallographic structures and different orientations. Here in this thesis we assumed that the polymer surface interaction makes a nonuniform surface due to the controlling of interaction on particular sites. Controlling the surface interactions lead to different orientations on the surface which is called facet. After understanding the rate of interactions of each facet we want to study the adsorption and activity rate of such a nonuniform crystal structure. We have studied three types orientation for catalyst such as striped, checkerboard and random to see whether the orientation affects the rate or not. Kinetically this is measured by the effective rate over the surface. The effective rate is related to the nature of the catalyst and the coverage of the surface by the reactant. Our result showed us under the same thermodynamic condition, the checkerboard nanostructure pattern has twice the effective reaction rate 
compared to the two other patterns. Investigating effective reaction rate by the KMC helps us to save the cost of using precious materials such as gold, titanium, platinum, etc, in experiments.

The second approach in this thesis was the influence of the polymer chain on crystal growth. Coating the surface with a polymer chain under certain conditions will help the surface to grow under specific circumstances.

In this thesis we applied a homopolymer chain in Chapter 5, and tried to understand the different phase transitions of the system based on different facet structures. This has been investigated by KMC simulation, which reveals the importance of the nanostructured pattern on phase transition of such a system. These results can be applied in many industries such as biomedical, optoelectrical, etc. In this Chapter 4 important phases have been explained. The first phase occurs at high temperatures; here the polymer chain is fully crumpled and desorbed on top of the surface, and due to the high fluctuation energy, it cannot adsorb on the surface. The second phase starts when a few monomers become adsorbed on the surface under a specific attractive pattern. This starting point differs from pattern to pattern as shown in our results. The striped pattern is the easiest pattern for adsorption among the other two nanostructured patterns, and checkerboard pattern is the most difficult pattern for adsorption due to the lowest number of degrees of freedom for the polymer chain conformation. Another important phase was pattern recognition of the surface by the polymer, after this phase, polymer will be frozen.

The final area examined in this thesis was copolymer adsorption. Our result can be broadly applied in many industries. The best example, which we explained frequently in this thesis, is when a block of polymer chain is attached to a molecule and makes a copolymer system. Once they reach the surface, the polymer plays an insulator role for some patterns and helps other slow energy facets grow under special condition. Architecting the order of different types of monomer (A and B) based on the different sizes of attractive sites was a challenge of this process.

Simulating such a system help the experimenters to save a great deal of material and time by designing the computationally efficient structure first. In several cases, our result has been focused on pattern structure and copolymer designing. Basically we studied 3 types of pattern, random, striped and checkerboard with three domain 
sizes 1,2,4. Furthermore we considered three types of copolymer chain A-alt-B on top of those surfaces. We compared these different phases in the previous chapter. In summary, we wanted to develop the most distinctive adsorption energy and pattern recognition between monomer type $\mathrm{A}$ and $\mathrm{B}$ in these three chains. We did indeed find the most differentiating patterns and chain for our thermodynamic conditions. This result showed the accuracy of the model. In future, we can apply different patterns by designing new polymer chains as well. 



\section{Appendix A}

\section{Homogenization theory in the}

\section{Limitation of a very small $\mathrm{L}$}

In this appendix, we consider the homogenization of equation (2.37) in the limit where the period $L$ becomes small compared to the far field. We introduce a function $\ell=\ell(x, y)$ which varies over the catalyst surface $\Gamma_{L}$. We assume that some appropriate boundary condition holds on some plane $\Gamma_{\text {far }}$ in the far field, but will neglect it for the purposes of the calculation. The boundary condition of interest is:

$$
-\ell(x, y) \vec{n} \cdot \nabla \rho=\rho, \text { on the catalyst surface } \Gamma_{L} .
$$

In weak form, with this boundary condition, equation (2.31) can be written:

$$
2 \int_{\Omega} \nabla \rho \cdot(\nabla u) d V+\int_{\Gamma_{L}} \frac{1}{\ell(x, y)} \rho u d \Gamma=0
$$

where $\Omega$ is the domain bounded by the parallel planes $\Gamma_{L}$ and $\Gamma_{\text {far }}$ and $u$ is an arbitrary test function. The homogenization limit is obtained when the period $L / H \rightarrow 0$, where $H$ is the far field distance. In this limit, one can consider a sequence of equations of the form of (A.2) with each corresponding to a surface $\Gamma_{L}$ with a smaller period $L$. In this limit, the second term weakly converges to its average over a single period:

$$
\int_{\Gamma_{L}} \frac{1}{\ell(x, y)} \rho u d \Gamma \rightarrow \int_{\Gamma_{0}}\left\langle\frac{1}{\ell(x, y)}\right\rangle \rho u d \Gamma
$$


The strong form of this homogenized boundary condition, which will hold in the limit $L \ll l \sim H$, is thus

$$
-\vec{n} \cdot \nabla \rho=\left\langle\frac{1}{\ell(x, y)}\right\rangle \rho, \text { on } \Gamma_{0}
$$

Thus, in terms of $k_{1}$, we can write equation (Eq. 2.24) in this limit as:

$$
-D \sqrt{\frac{2 \pi m}{k_{B} T}} \vec{n} \cdot \nabla \rho_{A}(r, t)=k_{e f f} \rho_{A},
$$

where

$$
k_{e f f}=\langle k(x, y)\rangle
$$




\section{Appendix B}

\section{Algorithm of Chapters 4,5,6}

We have developed different kinetic Monte Carlo methods for our system in different conditions. Here we explained different steps of MC processes:

\section{B.0.1 KMC Algorithm}

1. Set all the initial positions at $t=0$.

2. Assigne their rates and all their possible transitions.

3. Calculate total rate $\sum_{i=1}^{N} r_{i}=R$

4. Choose a random number between zero and one: $u=[0,1]$

5. Find an occurrence event at each time step by calculating: $r_{i-1}<u R<r_{i}$

6. Update all the positions and their related functions such as potentials.

7. Choose another random number to update the time step to $t+\tau, \tau=\frac{-\log u}{R}$.

8. Go back to the beginning of the loop.

\section{B.0.2 Kinetic MC algorithm of Homopolymer adsorption}

1. Set all the unit cells in a cubic box with length $\mathrm{L}=24$.

2. Distribute mesh sites on the bottom of the box.

3. Create a copolymer chain on top of the surface. 
4. Assign number of MC steps.

5. Choose a monomer randomly.

6. Count all the available states by considering the empty states and constrain the condition of the monomers with their nearest neighbors.

7. Choose one place randomly.

8. Check whether the selected monomer is close to either end or not.

9. If yes the current nearest neighbor will become the new end after conformation is approved.

10. Assign all the new places for the monomer and the rest of the chain.

11. Measure the current energy of state and the new places, energy of state.

12. Calculate the Rosenbluth weight function.

13. Choose a number randomly.

14. If it was within $\min \left\{1, \exp \beta\left(U_{f}-U_{i}\right)\right\}$, then it is accepted.

15. If the new place is a sticky site,check the penalties.

16. Exachange the old place for the new place.

17. Check the temperature transition for transiting the system to the new temperature

18. choose another random number.

19. If it was within $\min \left\{1+\exp \left[-U\left(q_{i}\right)\left(\beta_{m \pm 1}-\beta m\right)+\eta_{m \pm 1}-\eta m\right]\right\}$, then it is accepted.

20. Recalculate the probability of each subensembles.

\section{B.0.3 Kinetic MC algorithm of Copolymer adsorption}

1. Set all the unit cells in a cubic box with length $\mathrm{L}=24$.

2. Distrbute mesh sites on the bottom of the box.

3. Create a copolymer chain on top of the surface.

4. Assign the number of MC steps. 
5. Choose a monomer randomly.

6. Count all the available states by considering the empty states and the constrain condition of the monomers with their nearest neighbors.

7. Choose one place randomly.

8. Check whether the selected monomer is close to either end or not.

9. If yes, check whether the end is similar to its nearest neighbor or not.

10. If yes, the current nearest neighbor will become the new end after conformation is approved.

11. Assign all the new places for the monomer and the rest of the chain.

12. Measure the current energy of state and the new places, energy of state.

13. Calculate the Rosenbluth weight function.

14. Choose a number randomly.

15. If it was within $\min \left\{1, \exp \beta\left(U_{f}-U_{i}\right)\right\}$, then it is accepted.

16. If the new place is a sticky site, check the penalties.

17. Exchange the old place for the new place.

18. Check the temperature transition for transiting the system to the new temperature.

19. Choose another random number.

20. If it was within $\min \left\{1+\exp \left[-U\left(q_{i}\right)\left(\beta_{m \pm 1}-\beta m\right)+\eta_{m \pm 1}-\eta m\right]\right\}$, then it is accepted.

21. Recalculate the probability of each subensemble. 



\section{Appendix C}

\section{Adsorption concept}

In Chapter 1 and 2, we discuss the adsorption process. However, in many kinds of literature, we may have seen the absorption process as well. Sometimes these two terms are confusing. Therefore, in this appendix, we explain the difference between adsorption and absorption process.

\section{C.1 Adsorption versus Absorption}

What is the difference between adsorption and absorption? Imagine that a cup of coffee accidentally falls onto your office desk. What is the best way to dry it out? Perhaps two of the handiest tools that you would apply are ordinary paper and tissue paper, but which one works better? Obviously, ordinary paper cannot completely absorb the coffee however, tissue paper absorbs the liquid very well. Absorption is the transition of a substance throughout medium to form a solution, whereas adsorption is the adhesion and bonding of molecules which stick together and are weakly held by a substrate that can easily slip down from the surface. One of the best examples of the difference between adsorption and absorption is comparing the adhesion of perfume and french fries to your skin. Perfume is absorbed by our skin and it will disappear after a while, whereas the chips' oils will not be absorbed by your skin. The other difference between absorption and adsorption is that adsorption can be easily reversed by physical action such as motion, heating, etc. 



\section{Bibliography}

[1] ND Zhigadlo. Spontaneous growth of diamond from mnni solvent-catalyst using opposed anvil-type high-pressure apparatus. Journal of Crystal Growth, 395:1-4, 2014.

[2] Raoul Peltier. Biomimetic modification of crystal growth. PhD thesis, ResearchSpace@ Auckland, 2011.

[3] GH Nancollas and N Purdie. The kinetics of crystal growth. Quarterly Reviews, Chemical Society, 18(1):1-20, 1964.

[4] John William Mullin. Crystallization. Butterworth-Heinemann, 2001.

[5] PI Antonov and SP Nikanorov. Physical problems in crystal growth by the stepanov method. Journal of Crystal Growth, 50(1):3-7, 1980.

[6] Yuri N Palyanov, Alexander F Khokhryakov, Yuri M Borzdov, and Igor N Kupriyanov. Diamond growth and morphology under the influence of impurity adsorption. Crystal Growth \& Design, 13(12):5411-5419, 2013.

[7] Carl H Mc Murtry, WDG Boecher, SG Seshardri, JS Zanghi, and JE Garnier. Microstructure and material properties of sic-tib/sub 2/particulate composites. Am. Ceram. Soc. Bull (United States), 66(2), 1987.

[8] Andreas Caesalpinus. De metallicis libri tres. Recusi, curante Conrado Agricola.

[9] R Hengerer, B Bolliger, M Erbudak, and M Grätzel. Structure and stability of the anatase tio 2 (101) and (001) surfaces. Surface Science, 460(1):162-169, 2000.

[10] Xiaobing Liu, Xiaopeng Jia, Zhuangfei Zhang, Yong Li, Meihua Hu, Zhenxiang Zhou, and Hong-an Ma. Crystal growth and characterization of diamond from 
carbonyl iron catalyst under high pressure and high temperature conditions. Crystal Growth \& Design, 11(9):3844-3849, 2011.

[11] Carol Korzeniewski and Dawn Kardash. Use of a dynamic monte carlo simulation in the study of nucleation-and-growth models for co electrochemical oxidation. The Journal of Physical Chemistry B, 105(37):8663-8671, 2001.

[12] A Dkabrowski. Adsorption-from theory to practice. Advances in colloid and interface science, 93(1):135-224, 2001.

[13] Shaozhong Li, Qi Xiang, Dawen Wang, and Kang L Wang. Modeling of facet growth on patterned si substrate in gas source mbe. Journal of crystal growth, 157(1):185-189, 1995.

[14] Gerhard Ertl, H Knozinger, Jens Weitkamp, et al. Handbook of heterogeneous catalysis. VCH, 1997.

[15] Gerhard Ertl. Wilhelm ostwald: Founder of physical chemistry and nobel laureate 1909. Angewandte Chemie International Edition, 48(36):6600-6606, 2009 .

[16] Hugh S Taylor and Shou Chu Liang. The heterogeneity of catalyst surfaces for chemisorption. i. zinc oxide1. Journal of the American Chemical Society, 69(6): 1306-1312, 1947.

[17] Gert Ehrlich. The mechanism of chemisorption on metals. Journal of Physics and Chemistry of Solids, 1(1-2):3-13, 1956.

[18] JP Van der Eerden, C Van Leeuwen, P Bennema, WL Van der Kruk, and BP Th Veltman. Crystal growth: A comparison of monte carlo simulation nucleation and normal growth theories. Journal of Applied Physics, 48(6):2124-2130, 1977.

[19] Joseph L Katz and MARC D Donohue. A kinetic approach to homogeneous nucleation theory. Advances in Chemical Physics, 40:137-155, 1979.

[20] Dimitri D Vvedensky. Epitaxial nanostructures across length and time scales, 2002.

[21] Arup K Chakraborty and Aaron J Golumbfskie. Polymer adsorption-driven self-assembly of nanostructures. Annual review of physical chemistry, 52(1): $537-573,2001$. 
[22] Marc Husseman, Eva E Malmström, Molly McNamara, Mathew Mate, David Mecerreyes, Didier G Benoit, James L Hedrick, Paul Mansky, E Huang, Thomas P Russell, et al. Controlled synthesis of polymer brushes by "living" free radical polymerization techniques. Macromolecules, 32(5):1424-1431, 1999.

[23] PA Lee, G Said, R Davis, and TH Lim. On the optical properties of some layer compounds. Journal of Physics and Chemistry of Solids, 30(12):2719-2729, 1969.

[24] Hugh Stott Taylor. A theory of the catalytic surface. Proceedings of the Royal Society of London. Series A, Containing Papers of a Mathematical and Physical Character, 108(745):105-111, 1925.

[25] Henry Eyring. The activated complex in chemical reactions. The Journal of Chemical Physics, 3(2):107-115, 1935.

[26] Olaf Deutschmann. Modeling and simulation of heterogeneous catalytic reactions: from the molecular process to the technical system. John Wiley \& Sons, 2013.

[27] Keith J Laidler. The development of theories of catalysis. Archive for history of exact sciences, 35(4):345-374, 1986.

[28] Shannon S Stahl. Palladium oxidase catalysis: selective oxidation of organic chemicals by direct dioxygen-coupled turnover. Angewandte Chemie International Edition, 43(26):3400-3420, 2004.

[29] AJB Robertson. The early history of catalysis. Platinum Metals Rev, 19(2): 64-69, 1975.

[30] Michel Che and Carroll O Bennett. The influence of particle size on the catalytic properties of supported metals. Advances in Catalysis, 36:55-172, 1989.

[31] Michael J Saxton. Single-particle tracking: the distribution of diffusion coefficients. Biophysical journal, 72(4):1744, 1997.

[32] Olaf Deutschmann, Helmut Knözinger, Karl Kochloefl, and Thomas Turek. Heterogeneous catalysis and solid catalysts. Ullmann's Encyclopedia of Industrial Chemistry, 2009.

[33] J Bernsdorf, G Brenner, and F Durst. Numerical analysis of the pressure drop in porous media flow with lattice boltzmann (bgk) automata. Computer Physics Communications, 129(1):247-255, 2000. 
[34] Daniel Pedersen. Development of a kinetic monte carlo code. 2013.

[35] SB Charnley. Stochastic theory of molecule formation on dust. The Astrophysical Journal Letters, 562(1):L99, 2001.

[36] Bruce T Draine. Physics of the interstellar and intergalactic medium. Princeton University Press, 2010.

[37] Tim P Schulze. Efficient kinetic monte carlo simulation. Journal of Computational Physics, 227(4):2455-2462, 2008.

[38] Max J Hoffmann, Sebastian Matera, and Karsten Reuter. kmos: a lattice kinetic monte carlo framework. Computer Physics Communications, 185(7):2138-2150, 2014.

[39] Shin'ichi Matsumoto. Catalytic reduction of nitrogen oxides in automotive exhaust containing excess oxygen by nox storage-reduction catalyst. Cattech, 4 (2):102-109, 2000 .

[40] Justin B Siegel, Alexandre Zanghellini, Helena M Lovick, Gert Kiss, Abigail R Lambert, Jennifer L St Clair, Jasmine L Gallaher, Donald Hilvert, Michael H Gelb, Barry L Stoddard, et al. Computational design of an enzyme catalyst for a stereoselective bimolecular diels-alder reaction. Science, 329(5989):309-313, 2010.

[41] Larry E Overman. Mercury (ii)-and palladium (ii)-catalyzed [3, 3]-sigmatropic rearrangements [new synthetic methods (46)]. Angewandte Chemie International Edition in English, 23(8):579-586, 1984.

[42] S Khezrianjoo and HD Revanasiddappa. Langmuir-hinshelwood kinetic expression for the photocatalytic degradation of metanil yellow aqueous solutions by zno catalyst. Chemical Sciences Journal, 3:1, 2012.

[43] Kristen A Fichthorn and W Hh Weinberg. Theoretical foundations of dynamical monte carlo simulations. The Journal of chemical physics, 95(2):1090-1096, 1991.

[44] Axel Groß and Arezoo Dianat. Hydrogen dissociation dynamics on precovered pd surfaces: Langmuir is still right. Physical review letters, 98(20):206107, 2007.

[45] G Leofanti, G Tozzola, M Padovan, G Petrini, S Bordiga, and A Zecchina. Catalyst characterization: characterization techniques. Catalysis today, 34(3): 307-327, 1997. 
[46] Lothar Kunz, Lubow Maier, Steffen Tischer, and Olaf Deutschmann. Modeling the rate of heterogeneous reactions. ChemInform, 43(36):no, 2012.

[47] Michal Sabo, Antje Henschel, Heidrun Fröde, Elias Klemm, and Stefan Kaskel. Solution infiltration of palladium into mof-5: synthesis, physisorption and catalytic properties. Journal of Materials Chemistry, 17(36):3827-3832, 2007.

[48] Arthur W Adamson, Alice Petry Gast, et al. Physical chemistry of surfaces. 1967.

[49] James J Carberry. Chemical and catalytic reaction engineering. Courier Corporation, 2001.

[50] Kathleen Cornely-Moss. Kinetic theory of gases. J. Chem. Educ, 72(8):715, 1995.

[51] Richard P Feynman, Robert B Leighton, and Matthew Sands. The Feynman Lectures on Physics, Desktop Edition Volume I, volume 1. Basic books, 2013.

[52] Daniel T Gillespie. A general method for numerically simulating the stochastic time evolution of coupled chemical reactions. Journal of computational physics, 22(4):403-434, 1976.

[53] Tamas I Gombosi. Gaskinetic theory. Number 9. Cambridge University Press, 1994.

[54] Peter H Verdier and WH Stockmayer. Monte carlo calculations on the dynamics of polymers in dilute solution. The Journal of Chemical Physics, 36(1):227-235, 1962.

[55] SL Lin and JN Bardsley. Monte carlo simulation of ion motion in drift tubes. The Journal of Chemical Physics, 66(2):435-445, 1977.

[56] John Crank. The mathematics of diffusion. Oxford university press, 1979.

[57] Cesar Beleno and Kaven Yau. Computational physics project, universit at bonn, ws $10 / 11$.

[58] Paul A Webb. Introduction to chemical adsorption analytical techniques and their applications to catalysis. Micromeritics Instrument Corp. Technical Publications, 2003. 
[59] GR Belton. Langmuir adsorption, the gibbs adsorption isotherm, and interracial kinetics in liquid metal systems. Metallurgical Transactions B, 7(1):35-42, 1976.

[60] Mark F Granville. Student misconceptions in thermodynamics. J. Chem. Educ, $62(10): 847,1985$.

[61] Klaus Christmann. Introduction to surface physical chemistry, volume 1. Springer Science Business Media, 2013.

[62] Luis Caffarelli. The homogenization of surfaces and boundaries. Bulletin of the Brazilian Mathematical Society, New Series, 44(4):755-775, 2013.

[63] Nathaniel Joseph Lund. Effective slip lengths for stokes flow over rough, mixed-slip surfaces. 2015.

[64] Nat J Lund, Xingyou Philip Zhang, Keoni Mahelona, and Shaun C Hendy. Calculation of effective slip on rough chemically heterogeneous surfaces using a homogenization approach. Physical Review E, 86(4):046303, 2012.

[65] P Halevi, AA Krokhin, and J Arriaga. Photonic crystal optics and homogenization of 2d periodic composites. Physical review letters, 82(4):719, 1999.

[66] Chiang C Mei, Jean-Louis Auriault, and Chiu-On Ng. Some applications of the homogenization theory. Advances in applied mechanics, 32:277-348, 1996.

[67] JL Auriault. Heterogeneous medium. is an equivalent macroscopic description possible? International journal of engineering science, 29(7):785-795, 1991.

[68] S. C. Hendy and N. J. Lund. Effective slip boundary conditions for flows over nanoscale chemical heterogeneities. Phys. Rev. E, 76:066313, December 2007. doi: http://dx.doi.org/10.1103/PhysRevE.76.066313. URL http://journals.aps.org/pre/abstract/10.1103/PhysRevE.76.066313.

[69] Nat J. Lund, Xingyou Philip Zhang, Keoni Mahelona, and Shaun C. Hendy. Calculation of effective slip on rough chemically heterogeneous surfaces using a homogenization approach. Physical Review E, 86:046303, October 2012. doi: http://dx.doi.org/10.1103/PhysRevE.86.046303. URL http://journals.aps.org/pre/pdf/10.1103/PhysRevE.86.046303. 
[70] T. Zambelli, J. Wintterlin, J. Trost, and G. Ertl. Identification of the "active sites" of a surface-catalyzed reaction. Science, 273(5282):1688-1690, 1996. doi: 10.1126/science.273.5282.1688. URL http://www . sciencemag. org/content/299/5613/1688.

[71] Sang Hoon Joo, Jeong Y. Park, J. Russell Renzas, Derek R. Butcher, Wenyu Huang, and Gabor A. Somorjai. Previous article next article table of contents size effect of ruthenium nanoparticles in catalytic carbon monoxide oxidation. Nano Lett, 10(10 (7)), Jun 2010. doi: 10.1021/nl101700j. URL http://pubs.acs.org/doi/abs/10.1021/nl101700j.

[72] Haijun Zhang, Tatsuya Watanabe, Mitsutaka Okumura, Masatake Haruta, and Naoki Toshima. Catalytically highly active top gold atom on palladium nanocluster. Nature Materials, 11(11):49-52, October 2012. doi: $10.1038 /$ nmat3143.

[73] Alexis T. Bell. The impact of nanoscience on heterogeneous catalysis. Science, 299(5613):1688-1691, 2003. doi: 10.1126/science.1083671. URL http://www . sciencemag. org/content/299/5613/1688.

[74] Francisco J. Perez-Alonso, David N. McCarthy, Anders Nierhoff, Patricia Hernandez-Fernandez, Christian Strebel, Ifan E. L. Stephens, Jane H. Nielsen, and Ib Chorkendorff. The effect of size on the oxygen electroreduction activity of mass-selected platinum nanoparticles. Angewandte Chemie International Edition, 51(19):4641-4643, 2012. ISSN 1521-3773. doi: 10.1002/anie.201200586. URL http://dx.doi.org/10.1002/anie.201200586.

[75] Zhi-You Zhou, Na Tian, Jun-Tao Li, Ian Broadwell, and Shi-Gang Sun. Nanomaterials of high surface energy with exceptional properties in catalysis and energy storage. Chem. Soc. Rev., 40:4167-4185, 2011. doi: 10.1039/C0CS00176G. URL http://dx.doi.org/10.1039/COCS00176G.

[76] Thomas W. Hansen, Andrew T. DeLaRiva, Sivakumar R. Challa, and Abhaya K. Datye. Sintering of catalytic nanoparticles: Particle migration or ostwald ripening? Accounts of Chemical Research, 46(8):1720-1730, 2013. doi: 10.1021/ar3002427. URL http://dx.doi.org/10.1021/ar3002427. PMID: 23634641. 
[77] J M Flores-Camacho, J H Fischer-Wolfarth, M Peter, C T Campbell, S Schauermann, and H J Freund. Adsorption energetics of co on supported pd nanoparticles as a function of particle size by single crystal microcalorimetry. Phys Chem Chem Phys, 13:16800-16810, 2011. doi: 10.1039/c1cp21677e.

[78] Oleg M. Ilinitch, F.Petrus Cuperus, Ludmila V. Nosova, and Evgeni N. Gribov. Catalytic membrane in reduction of aqueous nitrates: operational principles and catalytic performance. Catalysis Today, 56(1-3):137 - 145, 2000. ISSN 0920-5861. doi: http://dx.doi.org/10.1016/S0920-5861(99)00270-9. URL http://www.sciencedirect.com/science/article/pii/S0920586199002709.

[79] Sydney Chapman and Thomas George Cowling. The mathematical theory of non-uniform gases: an account of the kinetic theory of viscosity, thermal conduction and diffusion in gases. Cambridge university press, 1970.

[80] Didier Astruc. Nanoparticles and catalysis. John Wiley \& Sons, 2008.

[81] Thomas Lee, Shaun C. Hendy, and Chiara Neto. Control of nanoparticle formation using the constrained dewetting of polymer brushes. Nanoscale, 7: 2894-2899, 2015. doi: 10.1039/C4NR07412B. URL http://dx.doi.org/10.1039/C4NR07412B.

[82] Runhai Ouyang, Jin-Xun Liu, and Wei-Xue Li. Atomistic theory of ostwald ripening and disintegration of supported metal particles under reaction conditions. Journal of the American Chemical Society, 135(5):1760-1771, 2013. doi: 10.1021/ja3087054. URL http://dx.doi.org/10.1021/ja3087054. PMID: 23272702 .

[83] J Evans and M Tromp. Interaction of small gas phase molecules with alumina supported rhodium nanoparticles: an in situ spectroscopic study. Journal of Physics: Condensed Matter, 20(18):184020, 2008. URL http://stacks.iop.org/0953-8984/20/i=18/a=184020.

[84] G. A. Tritsaris, J. Greeley, J. Rossmeisl, and J. K. Norskov. Atomic-scale modeling of particle size effects for the oxygen reduction reaction on pt. Catalysis Letters, 141:909-913, 2011. 
[85] Kourosh Malek Marc-Olivier Coppens. Dynamic monte-carlo simulations of diffusion limited reactions in rough nanopores. Chemical Engineering Science, 58:4787-4795, 2002. doi: 10.1016/j.ces.2002.11.001.

[86] Arthur F Voter. Introduction to the kinetic monte carlo method. In Radiation Effects in Solids, pages 1-23. Springer, 2007.

[87] Kurt Binder, Dieter Heermann, Lyle Roelofs, A John Mallinckrodt, Susan McKay, et al. Monte carlo simulation in statistical physics. Computers in Physics, 7(2):156-157, 1993.

[88] Sione Paea. Kinetic monte carlo (kmc) algorithm for nanocrystals. 2011.

[89] Hong Li, Yang Cao, Linda R Petzold, and Daniel T Gillespie. Algorithms and software for stochastic simulation of biochemical reacting systems. Biotechnology progress, 24(1):56-61, 2008.

[90] Peter Kratzer. Monte carlo and kinetic monte carlo methods. arXiv preprint arXiv:0904.2556, 2009.

[91] Eric Formo, Eric Lee, Dean Campbell, and Younan Xia. Functionalization of electrospun tio2 nanofibers with pt nanoparticles and nanowires for catalytic applications. Nano Letters, 8(2):668-672, 2008. doi: 10.1021/nl073163v. URL http://dx.doi.org/10.1021/nl073163v. PMID: 18205427.

[92] Michael Rubinstein. Polymer physics - the ugly duckling story: Will polymer physics ever become a part of "proper" physics? Journal of Polymer Science Part B: Polymer Physics, 48(24):2548-2551, 2010.

[93] CAJ Hoeve, EA DiMarzio, and P Peyser. Adsorption of polymer molecules at low surface coverage. The Journal of Chemical Physics, 42(7):2558-2563, 1965.

[94] PG De Gennes. Scaling theory of polymer adsorption. Journal de Physique, 37 (12):1445-1452, 1976.

[95] Akira Takahashi and Masami Kawaguchi. The structure of macromolecules adsorbed on interfaces. In Behavior of Macromolecules, pages 1-65. Springer, 1982. 
[96] A Silberberg. The adsorption of flexible macromolecules. part i. the isolated macromolecule at a plane interface1. The Journal of Physical Chemistry, 66(10): 1872-1883, 1962.

[97] Niklas Källrot. Dynamics of Polymer Adsorption onto Solid Surfaces in Good Solvent. Lund University, 2009.

[98] Matthias Wintermantel, Manfred Schmidt, Yasuhisa Tsukahara, Kanji Kajiwara, and Shinzo Kohjiya. Rodlike combs. Macromolecular rapid communications, 15 (3):279-284, 1994.

[99] P-G De Gennes. Some conformation problems for long macromolecules. Reports on Progress in Physics, 32(1):187, 1969.

[100] Justin Bois. Rudiments of polymer physics, 2002.

[101] Chinedum Osuji. Non-ideal chains: Size, statistics, free-energy.

[102] Masao Doi and Sam F Edwards. The theory of polymer dynamics, volume 73. oxford university press, 1988.

[103] A Silberberg. Theoretical aspects of the adsorption of macromolecules. In Journal of Polymer Science Part C: Polymer Symposia, volume 30, pages 393-397. Wiley Online Library, 1970.

[104] Sam F Edwards. The statistical mechanics of polymers with excluded volume. Proceedings of the Physical Society, 85(4):613, 1965.

[105] Somendra M Bhattacharjee, Achille Giacometti, and Amos Maritan. Flory theory for polymers. Journal of Physics: Condensed Matter, 25(50):503101, 2013.

[106] Paul Flory, M Volkenstein, et al. Statistical mechanics of chain molecules, 1969.

[107] Andrey Milchev and Kurt Binder. Static and dynamic properties of adsorbed chains at surfaces: Monte carlo simulation of a bead-spring model. Macromolecules, 29(1):343-354, 1996.

[108] SF Edwards. The theory of polymer solutions at intermediate concentration. Proceedings of the Physical Society, 88(2):265, 1966. 
[109] Yongmei Wang and Iwao Teraoka. Computer simulation of semidilute polymer solutions in confined geometry: pore as a microscopic probe. Macromolecules, 30 (26):8473-8477, 1997.

[110] Joshua N Milstein and Jens-Christian Meiners. Worm-like chain (wlc) model. In Encyclopedia of Biophysics, pages 2757-2760. Springer, 2013.

[111] Jason De Joannis. Equilibrium properties of polymer solutions at surfaces: Monte Carlo simulations. PhD thesis, University of Florida, 2000.

[112] Kerson Huang. Introduction to statistical physics. CRC Press, 2009.

[113] Kiyosi Ito. Diffusion Processes. Wiley Online Library, 1974.

[114] Eugene Helfand. Theory of inhomogeneous polymers: Fundamentals of the gaussian random-walk model. The Journal of Chemical Physics, 62(3):999-1005, 1975.

[115] Hagen Kleinert. Path integrals in quantum mechanics, statistics, polymer physics, and financial markets. World Scientific, 2009.

[116] Pierre-Gilles de Gennes. Exponents for the excluded volume problem as derived by the wilson method. Physics Letters A, 38(5):339-340, 1972.

[117] CAJ Hoeve. On the general theory of polymer absorption at a surface. In Journal of Polymer Science Part C: Polymer Symposia, volume 30, pages 361-367. Wiley Online Library, 1970.

[118] Witold Brostow, Hamide Ertepinar, and RP Singh. Flow of dilute polymer solutions: chain conformations and degradation of drag reducers. Macromolecules, 23(24):5109-5118, 1990.

[119] Barry D Hughes. Random walks and random environments. 1996.

[120] F Brochard and Pierre-Gilles de Gennes. Dynamics of confined polymer chains. The Journal of Chemical Physics, 67(1):52-56, 1977.

[121] Graham Smith. The measurement of free energy by monte carlo computer simulation. 1996. 
[122] Nobuyuki Nakajima, Junichi Higo, Akinori Kidera, and Haruki Nakamura. Free energy landscapes of peptides by enhanced conformational sampling. Journal of molecular biology, 296(1):197-216, 2000.

[123] Christophe Chipot and Andrew Pohorille. Free energy calculations. Springer, 2007.

[124] MP Seah and WA Dench. Quantitative electron spectroscopy of surfaces: a standard data base for electron inelastic mean free paths in solids. Surface and interface analysis, 1(1):2-11, 1979.

[125] Marc Souaille and Benoit Roux. Extension to the weighted histogram analysis method: combining umbrella sampling with free energy calculations. Computer physics communications, 135(1):40-57, 2001.

[126] W Keith Hastings. Monte carlo sampling methods using markov chains and their applications. Biometrika, 57(1):97-109, 1970.

[127] AP Lyubartsev, AA Martsinovski, SV Shevkunov, and PN Vorontsov-Velyaminov. New approach to monte carlo calculation of the free energy: Method of expanded ensembles. The Journal of chemical physics, 96(3): 1776-1783, 1992.

[128] Pascal Viot. Numerical simulation in statistical physics lecture in master 2 "physics of complex systems" and "modeling, statistics and algorithms for out-of-equilibrium systems. 2015.

[129] Ulrich HE Hansmann and Yuko Okamoto. New monte carlo algorithms for protein folding. Current opinion in structural biology, 9(2):177-183, 1999.

[130] David Sherrington and Scott Kirkpatrick. Solvable model of a spin-glass. Physical review letters, 35(26):1792, 1975.

[131] Martien A Cohen Stuart, Terence Cosgrove, and Brian Vincent. Experimental aspects of polymer adsorption at solid/solution interfaces. Advances in Colloid and Interface Science, 24:143-239, 1985.

[132] Sanjay S Patel and Matthew Tirrell. Measurement of forces between surfaces in polymer fluids. Annual Review of Physical Chemistry, 40(1):597-635, 1989. 
[133] Arup K Chakraborty and Matthew Tirrell. Polymer adsorption. MRS Bulletin, 21(01):28-32, 1996.

[134] Julie Melissa Goddard and JH Hotchkiss. Polymer surface modification for the attachment of bioactive compounds. Progress in polymer science, 32(7):698-725, 2007.

[135] Yuji Sugita and Yuko Okamoto. Free-energy calculations in protein folding by generalized-ensemble algorithms. In Computational Methods for Macromolecules: Challenges and Applications, pages 304-332. Springer, 2002.

[136] Per Linse and Niklas Kallrot. Polymer adsorption from bulk solution onto planar surfaces: effect of polymer flexibility and surface attraction in good solvent. Macromolecules, 43(4):2054-2068, 2010.

[137] Debabrata Panja, Gerard T Barkema, and Anatoly B Kolomeisky. Non-equilibrium dynamics of single polymer adsorption to solid surfaces. Journal of Physics: Condensed Matter, 21(24):242101, 2009.

[138] Aaron J Golumbfskie, Vijay S Pande, and Arup K Chakraborty. Simulation of biomimetic recognition between polymers and surfaces. Proceedings of the National Academy of Sciences, 96(21):11707-11712, 1999.

[139] W Gottstein, S Kreitmeier, M Wittkop, D Goritz, and F Gotsis. Monte carlo simulations of the adsorption of a single polymer chain on rough surfaces. Polymer, 38(7):1607-1613, 1997.

[140] Eiji Yashima, Katsuhiro Maeda, and Tatsuya Nishimura. Detection and amplification of chirality by helical polymers. Chemistry-A European Journal, 10 (1):42-51, 2004.

[141] Matthew Moffitt and Adi Eisenberg. Size control of nanoparticles in semiconductor-polymer composites. 1. control via multiplet aggregation numbers in styrene-based random ionomers. Chemistry of Materials, 7(6):1178-1184, 1995.

[142] Anton Ginzburg, Tibor Macko, Volker Dolle, and Robert Brüll. Characterization of polyolefins by comprehensive high-temperature two-dimensional liquid chromatography (ht 2d-lc). European Polymer Journal, 47(3):319-329, 2011. 
[143] T Cosgrove, T Mc Obey, and B Vincent. The configuration of sodium poly (styrene sulfonate) at polystyrene/solution interfaces. Journal of colloid and interface science, 111(2):409-418, 1986.

[144] Chris Oostenbrink, MMH van Lipzig, and Wilfred F van Gunsteren. Applications of molecular dynamics simulations in drug design. Comprehensive Medicinal Chemistry II, 4:651-668, 2007.

[145] Hiroshi Maeda. Smancs and polymer-conjugated macromolecular drugs: advantages in cancer chemotherapy. Advanced drug delivery reviews, 46(1): 169-185, 2001.

[146] Kajari Kargupta and Ashutosh Sharma. Morphological self-organization by dewetting in thin films on chemically patterned substrates. The Journal of chemical physics, 116(7):3042-3051, 2002.

[147] Hongbo Zeng. Polymer adhesion, friction, and lubrication. John Wiley \& Sons, 2013.

[148] Robert Simha, HL Frisch, and FR Eirich. The adsorption of flexible macromolecules. The Journal of Physical Chemistry, 57(6):584-589, 1953.

[149] EA DiMarzio, CM Guttman, and JD Hoffman. Calculation of lamellar thickness in a diblock copolymer, one of whose components is crystalline. Macromolecules, 13(5):1194-1198, 1980.

[150] SV Shevkunov. High temp. phys.(ussr) 1988, 26, 246. shevkunov, sv; vorontsov-velyaminov, pn; martsinovskii, aa. Mol. Simul, 5:119, 1991.

[151] Paul J Flory. Thermodynamics of high polymer solutions. The Journal of chemical physics, 10(1):51-61, 1942.

[152] Kurt Kremer and Kurt Binder. Monte carlo simulation of lattice models for macromolecules. Computer Physics Reports, 7(6):259-310, 1988.

[153] A Sharma and G Reiter. Morphological phase transitions in spontaneous dewetting of thin films on homogeneous and heterogeneous surfaces. Phase Transitions, 75(4-5):377-399, 2002. 
[154] Mitra Tavakoli, Ali Asghar Katbab, and Hossein Nazockdast. Nr/sbr/organoclay nanocomposites: Effects of molecular interactions upon the clay microstructure and mechano-dynamic properties. Journal of Applied Polymer Science, 123(3): 1853-1864, 2012.

[155] Paul J Flory. Phase equilibria in solutions of rod-like particles. In Proceedings of the Royal Society of London A: Mathematical, Physical and Engineering Sciences, volume 234, pages 73-89. The Royal Society, 1956.

[156] Mike P Allen and Dominic J Tildesley. Computer simulation of liquids. Oxford university press, 1989.

[157] Peter Frantz and Steve Granick. Kinetics of polymer adsorption and desorption. Physical review letters, 66(7):899, 1991.

[158] Ksharesh Dutta Dubey and Rajendra Prasas Ojha. molecular dynamics simulations: applicability and scopes in computational biochemistry. Nanoscience and Computational Chemistry: Research Progress, page 371, 2013.

[159] E Eisenriegler, K Kremer, and K Binder. Adsorption of polymer chains at surfaces: scaling and monte carlo analyses. The Journal of Chemical Physics, 77 (12):6296-6320, 1982.

[160] David Chandler. Introduction to modern statistical mechanics. Introduction to Modern Statistical Mechanics, by David Chandler, pp. 288. Foreword by David Chandler. Oxford University Press, Sep 1987. ISBN-10: 0195042778. ISBN-13: 97801950427r71, 1, 1987.

[161] Theodore W Burkhardt and Ihnsouk Guim. Free energy of a long, flexible, self-avoiding polymer chain in a tube. Physical Review E, 59(5):5833, 1999.

[162] PN Vorontsov-Velyaminov, AV Broukhno, TV Kuznetsova, and AP Lyubartsev. Free energy calculations by expanded ensemble method for lattice and continuous polymers. The Journal of Physical Chemistry, 100(4):1153-1158, 1996.

[163] AP Lyubartsev, OK Førrisdahl, and A Laaksonen. Calculations of solvation free energies by expanded ensemble method. In 2nd International Conference on Natural Gas Hydrates. Toulouse, France, June, pages 2-6. Citeseer, 1996. 
[164] Qiliang Yan and Juan J de Pablo. Hyper-parallel tempering monte carlo: Application to the lennard-jones fluid and the restricted primitive model. The Journal of chemical physics, 111(21):9509-9516, 1999.

[165] Alexander P Lyubartsev, Jay X Tang, Paul A Janmey, and Lars Nordenskiöld. Electrostatically induced polyelectrolyte association of rodlike virus particles. Physical review letters, 81(24):5465, 1998.

[166] Koji Hukushima, Hajime Takayama, and Koji Nemoto. Application of an extended ensemble method to spin glasses. International Journal of Modern Physics C, 7(03):337-344, 1996.

[167] John P Valleau and L Kenneth Cohen. Primitive model electrolytes. i. grand canonical monte carlo computations. The Journal of chemical physics, 72(11): 5935-5941, 1980.

[168] Mihaly Mezei. Calculation of solvation free-energy differences for large solute change from computer simulations with quadrature-based nearly linear thermodynamic integration. Molecular simulation, 10(2-6):225-239, 1993.

[169] M Lal, MA Turpin, KA Richardson, and D Spencer. Configurational behavior of isolated chain molecules adsorbed from athermal solutions. Adv Chem Se, 8: 16-27, 1975.

[170] Peter Grassberger. Pruned-enriched rosenbluth method: Simulations of $\theta$ polymers of chain length up to 1000 000. Physical Review E, 56(3):3682, 1997.

[171] Agnieszka Manka, Waldemar Nowicki, and Grazyna Nowicka. Monte carlo simulations of a polymer chain conformation. the effectiveness of local moves algorithms and estimation of entropy. Journal of molecular modeling, 19(9): 3659-3670, 2013.

[172] James J Semler and Jan Genzer. Monte carlo simulations of copolymer adsorption at planar chemically patterned surfaces: Effect of surface domain sizes. The Journal of chemical physics, 119(10):5274-5280, 2003.

[173] Collin D Wick and J Ija Siepmann. Self-adapting fixed-end-point configurational-bias monte carlo method for the regrowth of interior segments of 
chain molecules with strong intramolecular interactions. Macromolecules, 33(19): 7207-7218, 2000.

[174] Hong Li, Bin Gong, Chang-Ji Qian, and Meng-Bo Luo. Critical adsorption of a flexible polymer on a stripe-patterned surface. Soft matter, 11(16):3222-3231, 2015.

[175] Alexey A Polotsky. Adsorption of a homopolymer chain onto a heterogeneous stripe-patterned surface studied using a directed walk model of the polymer. Journal of Physics A: Mathematical and Theoretical, 49(1):015001, 2015.

[176] Alexey Polotsky, Friederike Schmid, and Andreas Degenhard. Polymer adsorption onto random planar surfaces: Interplay of polymer and surface correlations. The Journal of chemical physics, 121(10):4853-4864, 2004.

[177] Arthi Jayaraman, Carol K Hall, and Jan Genzer. Designing pattern-recognition surfaces for selective adsorption of copolymer sequences using lattice monte carlo simulation. Physical review letters, 94(7):078103, 2005.

[178] Juan J Cerda and Tomás Sintes. Stiff polymer adsorption. onset to pattern recognition. Biophysical chemistry, 115(2):277-283, 2005.

[179] Juan Rodriguez-Hernandez and Aitziber L Cortajarena. Selective biorecognition on polymer surfaces: Remarks and future trends. In Design of Polymeric Platforms for Selective Biorecognition, pages 387-389. Springer, 2015.

[180] GJ Fleer, JMHM Scheutjens, and MA Cohen Stuart. Theoretical progress in polymer adsorption, steric stabilization and flocculation. Colloids and Surfaces, $31: 1-29,1988$.

[181] Dieter Hofmann, Lydia Fritz, Jens Ulbrich, Claudia Schepers, and Martin Bohning. Detailed-atomistic molecular modeling of small molecule diffusion and solution processes in polymeric membrane materials. Macromolecular theory and simulations, 9(6):293-327, 2000.

[182] Dirk Schmaljohann. Thermo-and ph-responsive polymers in drug delivery. Advanced drug delivery reviews, 58(15):1655-1670, 2006. 
[183] Srinath Cheluvaraja and Hagai Meirovitch. Calculation of the entropy and free energy from monte carlo simulations of a peptide stretched by an external force. The Journal of Physical Chemistry B, 109(46):21963-21970, 2005.

[184] Jan Genzer. Self-consistent field study of copolymer adsorption at planar chemically rough surfaces: an interplay between the substrate chemical pattern and copolymer sequence distribution. Advances in Colloid and Interface Science, 94(1):105-134, 2001.

[185] Lennart Lindfors, Sara Forssen, Jan Westergren, and Ulf Olsson. Nucleation and crystal growth in supersaturated solutions of a model drug. Journal of colloid and interface science, 325(2):404-413, 2008.

[186] Yury A Kriksin, Pavel G Khalatur, and Alexei R Khokhlov. Adsorption of multiblock copolymers onto a chemically heterogeneous surface: a model of pattern recognition. The Journal of chemical physics, 122(11):114703, 2005.

[187] Jan Genzer. Copolymer adsorption on planar substrates with a random distribution of chemical heterogeneities. The Journal of Chemical Physics, 115 (10):4873-4882, 2001.

[188] Miri Park, Christopher Harrison, Paul M Chaikin, Richard A Register, and Douglas H Adamson. Block copolymer lithography: periodic arrays of 1011 holes in 1 square centimeter. Science, 276(5317):1401-1404, 1997.

[189] Frédéric A Denis, Antoine Pallandre, Bernard Nysten, Alain M Jonas, and Christine C Dupont-Gillain. Alignment and assembly of adsorbed collagen molecules induced by anisotropic chemical nanopatterns. Small, 1(10):984-991, 2005.

[190] II Potemkin, E Yu Kramarenko, AR Khokhlov, RG Winkler, P Reineker, P Eibeck, JP Spatz, and M Möller. Nanopattern of diblock copolymers selectively adsorbed on a plane surface. Langmuir, 15(21):7290-7298, 1999.

[191] Dietrich Braun, Harald Cherdron, Matthias Rehahn, Helmut Ritter, and Brigitte Voit. Polymer synthesis: theory and practice: fundamentals, methods, experiments. Springer Science \& Business Media, 2012. 